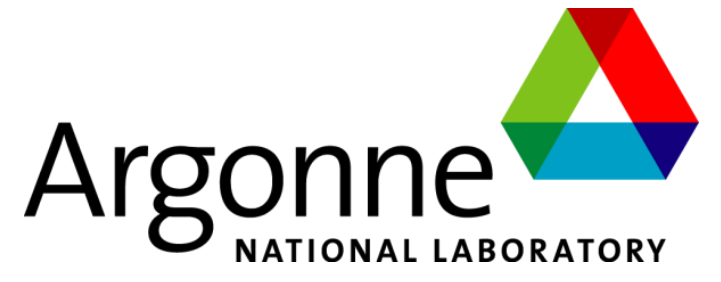

ANL/NE-17/14

\title{
Development of a Computational Approach to Detect Instability and Incipient Motion of Large Riprap Rocks
}




\title{
About Argonne National Laboratory
}

Argonne is a U.S. Department of Energy laboratory managed by UChicago Argonne, LLC under contract DE-AC02-06CH11357. The Laboratory's main facility is outside Chicago, at 9700 South Cass Avenue, Argonne, Illinois 60439. For information about Argonne and its pioneering science and technology programs, see www.anl.gov.

\section{DOCUMENT AVAILABILITY}

Online Access: U.S. Department of Energy (DOE) reports produced after 1991 and a growing number of pre-1991 documents are available free via DOE's SciTech Connect (http://www.osti.gov/scitech/)

\author{
Reports not in digital format may be purchased by the public from the \\ National Technical Information Service (NTIS): \\ U.S. Department of Commerce \\ National Technical Information Service \\ 5301 Shawnee Rd \\ Alexandra, VA 22312 \\ www.ntis.gov \\ Phone: (800) 553-NTIS (6847) or (703) 605-6000 \\ Fax: (703) 605-6900 \\ Email: orders@ntis.gov \\ Reports not in digital format are available to DOE and DOE contractors from the \\ Office of Scientific and Technical Information (OSTI): \\ U.S. Department of Energy \\ Office of Scientific and Technical Information \\ P.O. Box 62 \\ Oak Ridge, TN 37831-0062 \\ www.osti.gov \\ Phone: (865) 576-8401 \\ Fax: (865) 576-5728 \\ Email: reports@osti.gov
}

Disclaimer

This report was prepared as an account of work sponsored by an agency of the United States Government. Neither the United States Government nor any agency thereof, nor UChicago Argonne, LLC, nor any of their employees or officers, makes any warranty, express or implied, or assumes any legal liability or responsibility for the accuracy, completeness, or usefulness of any information, apparatus, product, or process disclosed, or represents that its use would not infringe privately owned rights. Reference herein to any specific commercial product, process, or service by trade name, trademark, manufacturer, or otherwise, does not necessarily constitute or imply its endorsement, recommendation, or favoring by the United States Government or any agency thereof. The views and opinions of document authors expressed herein do not necessarily state or reflect those of the United States Government or any agency thereof, Argonne National Laboratory, or UChicago Argonne, LLC. 


\section{Development of a Computational Approach to Detect Instability and Incipient Motion of Large Riprap Rocks}

by

C. Bojanowski, and S.A. Lottes

Transportation Research and Analysis Computing Center (TRACC)

Nuclear Engineering Division, Argonne National Laboratory

K. Flora

California Department of Transportation, Sacramento, California, USA

O. Suaznabar, J. Shen

Genex Systems-TFHRC, McLean, Virginia, USA

K. Kerenyi

Turner-Fairbank Highway Research Center

Federal Highway Administration

U.S. Department of Transportation

August 2017 


\section{Table of Contents}

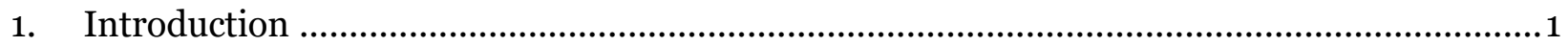

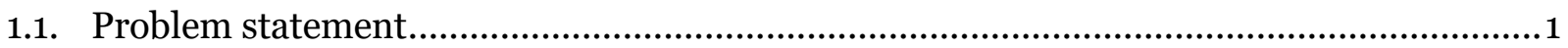

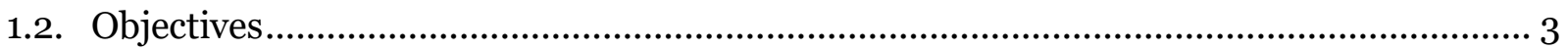

2. Coupling Methodologies for Solving FSI Problems ...................................................... 5

2.1. Governing Equations for the Fluid and Structural Domains ....................................... 5

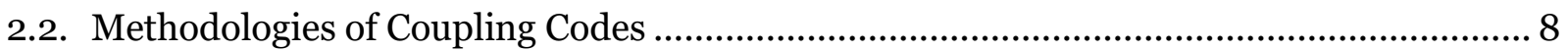

2.3. Workflow of the File Based Data Exchange with Explicit Coupling ................................. 9

2.4. Challenges, Limitations and Alternative Solutions of Current Implementation ..............14

2.5. Application of the Methodology to a Flat Bed Case ......................................................18

3. Verification of the Coupling Mechanism through comparison with a STAR-CCM+/Abaqus Benchmark FSI Problems .................................................................................... 24

4. Validation of the Methodology ................................................................................... 27

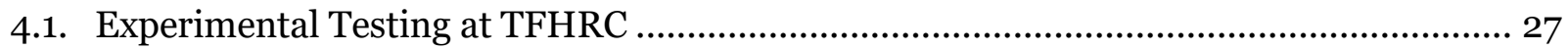

4.1.1. Geometry of the Test Section ....................................................................... 27

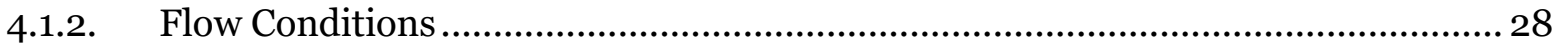

4.1.3. Equilibrium Scour of Riprap Apron ............................................................. 28

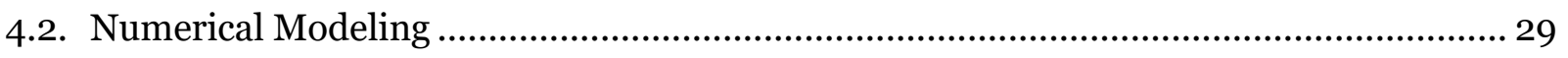

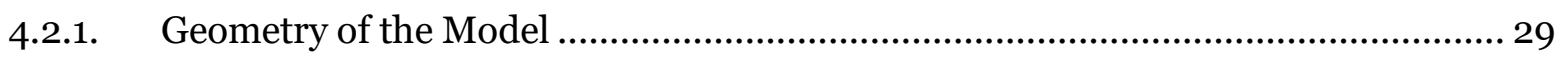

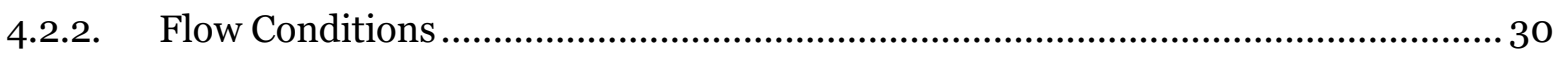

4.2.3. Model Development..................................................................................... 30

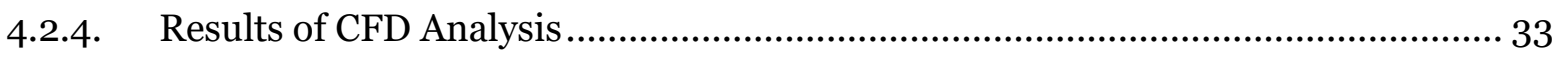

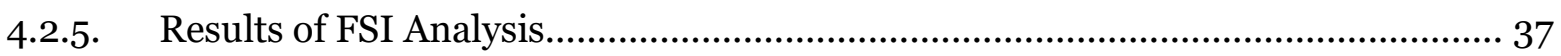

5. Application of FSI Coupling to Analysis of the Middle Fork Feather River Bridge Riprap

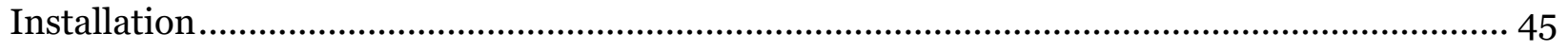

5.1. Site Conditions and Riprap Risk Assessment........................................................ 45

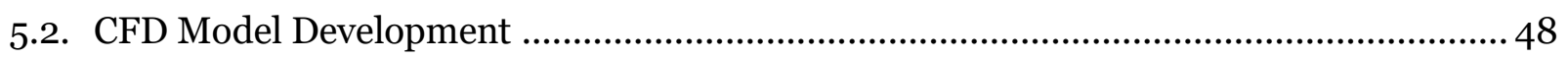

5.3. Comparison of Flow Conditions with 2D Analysis .................................................. 50

5.4. Riprap Geometry Development ........................................................................... 53

5.5. Finite Element Method Model Development ......................................................... 58

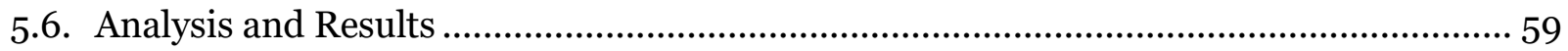

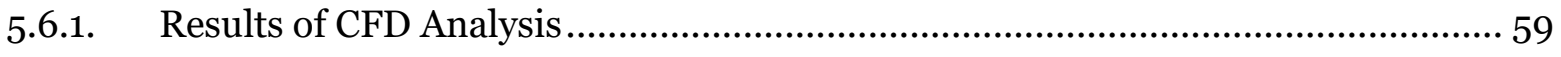




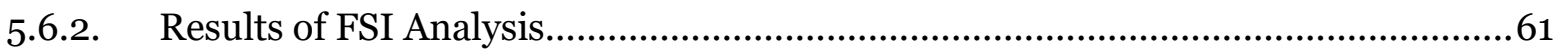

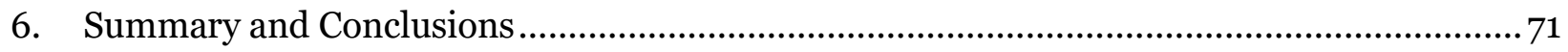

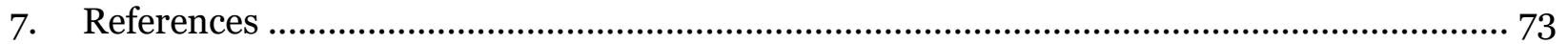




\section{List of Figures}

Figure 1.1: Historic, realigned and current river channel alignment [1] .......................................

Figure 1.2 Comparison of riprap sizing curves at a rectangular pier based on [3]......................... 2

Figure 2.1: Initial stage of the FSI problem and an intermediate stage with mesh morphing....... 5

Figure 2.2: Definition of the domains for analysis of riprap stability as an FSI problem ................7

Figure 2.3: Loose and strong FSI coupling schemes ................................................................... 9

Figure 2.4: Current implementation of coupling workflow between STAR-CCM+ and LS-DYNA

Figure 2.5: Mesh distortion as a result of morphing .....................................................................12

Figure 2.6: Remeshed domain after failure of the mesh morpher ................................................12

Figure 2.7 Stages in the coupled simulation for water induced rock motion ..................................13

Figure 2.8: A rock surface and feature curves created on all edges of the mesh ............................13

Figure 2.9: Definition of separate subregions for each rock............................................................15

Figure 2.10: Overset meshes in the main domain and the subdomain with the moving rock .....16

Figure 2.11: New implementation of coupling workflow between STAR-CCM+ and LS-DYNA

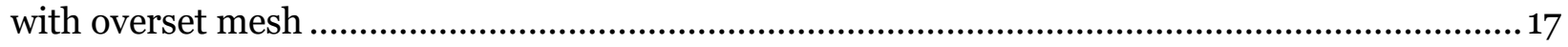

Figure 2.12: Forces in the coupling with overset mesh.................................................................... 17

Figure 2.13: Point cloud of a scanned rock and its triangulated representation..............................19

Figure 2.14: Geometry of the CFD model (a) layout 1, side view (b) layout 1, top view (c) layout 2,

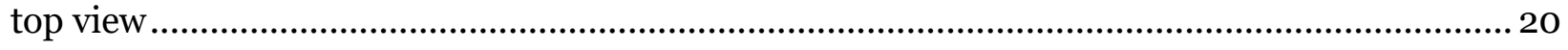

Figure 2.15: Snapshots from simulation for layout 1 and inlet velocity of $3.0 \mathrm{~m} / \mathrm{s}$....................... 22

Figure 2.16: Snapshots from simulation for layout 2 and inlet velocity of $3.0 \mathrm{~m} / \mathrm{s}$...................... 23

Figure 3.1: CFD domain and grid for analysis of flexible plate protruding into the flow setup for FSI analysis coupling with LS-DYNA ................................................................................... 24

Figure 3.2: Morphed mesh at maximum plate deflection in (a) ABAQUS coupling (b) LS-DYNA

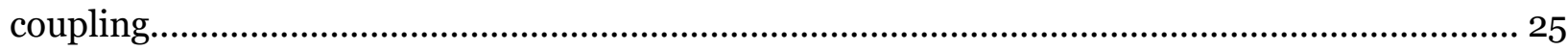

Figure 3.3: Time history of the plate deflection in (left) LS-DYNA - STAR-CCM+ coupling (right)

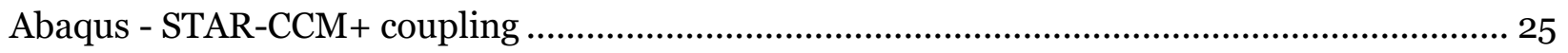


Figure 3.4: Velocity vector plot in the middle plane at maximum plate deflection .................... 26

Figure 3.5: Pressure distribution in the middle plane at maximum plate deflection ................. 26

Figure 4.1: Plan View of Test Section................................................................................ 27

Figure 4.2: Installation of riprap layers (left) first layer (right) second layer ........................... 28

Figure 4.3: Scanned Bathymetry of rock layout after test was finished ......................................... 29

Figure 4.4: Failure of riprap at the upstream corner ............................................................... 29

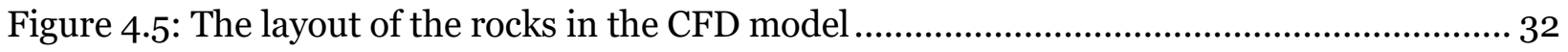

Figure 4.6: Pre drop and final position of the movable rocks ............................................... 33

Figure 4.7: Velocity profile in a cross section just above the riprap rocks for the case with inlet velocity of $1.3 \mathrm{~m} / \mathrm{s}$ in closed lid simulation.......................................................................... 34

Figure 4.8: Velocity profile in a cross section just above the riprap rocks for the case with inlet

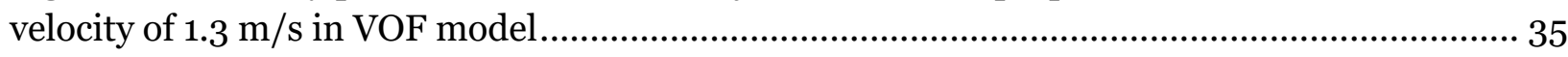

Figure 4.9: Water surface location for the VOF and closed lid simulation .............................. 35

Figure 4.10: Location of the rocks with the highest forces in CFD analysis.............................. 36

Figure 4.11: Forces on the moveable rocks in the simulation with inlet velocity of $1.3 \mathrm{~m} / \mathrm{s}$........ 37

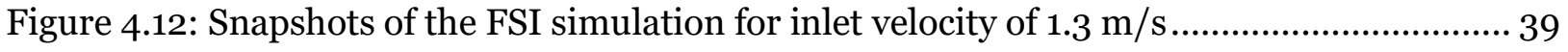

Figure 4.13: Position of rocks at $2.0 \mathrm{sec}$ in FSI simulation for inlet velocity of $1.3 \mathrm{~m} / \mathrm{s} \ldots \ldots \ldots \ldots . . .40$

Figure 4.14: Position of rocks at $2.0 \mathrm{sec}$ in the FSI simulation for inlet velocity of $1.4 \mathrm{~m} / \mathrm{s}$........ 40

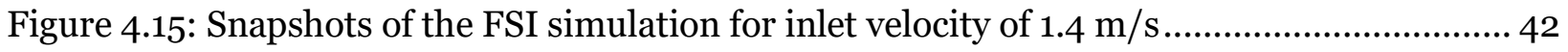

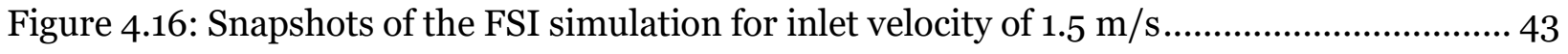

Figure 4.17: Position of rocks at 2.0 sec in the FSI simulation for inlet velocity of $1.5 \mathrm{~m} / \mathrm{s} \ldots \ldots . . .44$

Figure 5.1: Boat survey and GPS points in the bridge vicinity [1] ........................................ 45

Figure 5.2: Bathymetry of the river before installation of the riprap (2011) [1] ....................... 45

Figure 5.3: Designed rock layout for the pier protection [1] ….............................................. 46

Figure 5.4: 2013 Bathymetry of the river after the riprap installation (2013) [1] ..................... 46

Figure 5.5: Riprap installation near Pier-3 in August of 2012 [1] ........................................... 47

Figure 5.6: Definition of the bed boundaries in the CFD model ............................................. 48 
Figure 5.7: Surface mesh of the riverbed in CFD model.............................................................. 49

Figure 5.8: Cross section through the finite volume mesh used in STAR-CCM+ model .............. 50

Figure 5.9: Cross section through the finite volume of subregion used in FSI computations. .... 50

Figure 5.10: Depth averaged velocities in the 2D TUFLOW calculation.........................................51

Figure 5.11: Water surface velocity in $3 \mathrm{D}$ calculation with Star-CCM+ ...................................... 52

Figure 5.12: Perspective view of the water surface near the bridge with overlaid velocity ........... 52

Figure 5.13: Perspective view of the water surface level under the bridge with overlaid relative height .................................................................................................................................... 52

Figure 5.14: Depth averaged velocity under the bridge in 2D and 3D calculations ...................... 53

Figure 5.15: Design drawings of the riprap around Pier-3 of the bridge......................................... 54

Figure 5.16: Geometry of the riprap used in the CFD model.......................................................... 54

Figure 5.17: Pictures of riprap installation near Pier-3 during and after the construction........... 55

Figure 5.18: Top view on the installed riprap ………................................................................ 56

Figure 5.19: Front view on the pier with installed riprap ............................................................. 56

Figure 5.20: The extent of the riprap derived from the bed scan....................................................57

Figure 5.21: Extent of the riprap in the original CFD model.......................................................57

Figure 5.22: Extent of the riprap in the updated CFD model with movable rocks ........................ 58

Figure 5.23: Placement of moveable rocks around the pier............................................................ 59

Figure 5.24: Forces on the moveable rocks in the simulation with discharge of 30,100 cfs (100 year flood) .............................................................................................................................. 60

Figure 5.25: Velocity vectors on the interfaces between the scour subregion and the global

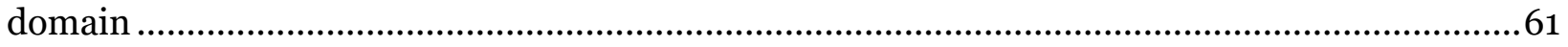

Figure 5.26: Velocity vectors on the plane just above the bed rocks .............................................62 62

Figure 5.27: Snapshots of the FSI simulation for Q100 discharge (11.1 ft/s average inlet velocity)

Figure 5.28: Trajectories of the moving rocks for the case Q100 discharge (11.1 ft/s average inlet velocity) 64

Figure 5.29: Water surface level in the simulation of $1.6{ }^{*}$ Q100 conditions ................................. 65

Figure 5.30: Raised water surface level by the inlet in the simulation of $1.6{ }^{*}$ Q100 conditions. 65 
Figure 5.31: Snapshots of the FSI simulation for $1.1{ }^{*}$ Q100 discharge (12.2 ft/s average inlet

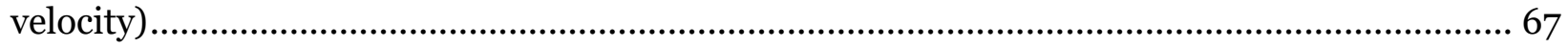

Figure 5.32: Trajectories of the moving rocks for the case $1.1 *$ Q100 discharge $(12.2 \mathrm{ft} / \mathrm{s}$ average

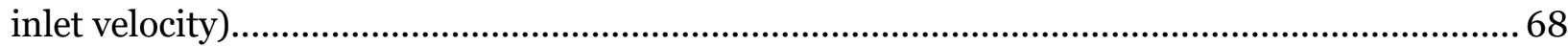

Figure 5.33: Trajectories of the moving rocks for the case $1.2 *$ Q100 discharge $(13.3 \mathrm{ft} / \mathrm{s}$ average

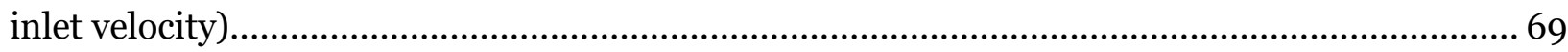

Figure 5.34: Snapshots of the FSI simulation for $1.2 *$ Q10o discharge (13.3 ft/s average inlet

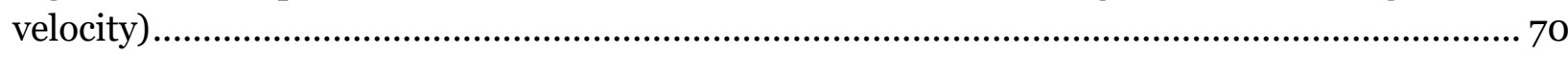




\section{List of Tables}

Table 2.1: Motion of the rocks in two analyzed cases .............................................................21

Table 4.1: Flow conditions for experiments...................................................................... 28

Table 4.2: Flow conditions based on Froude similarity …..................................................... 30

Table 4.3: Forces on the critical rocks in the simulation with inlet velocity of $1.3 \mathrm{~m} / \mathrm{s} \ldots \ldots \ldots \ldots . . . .38$

Table 4.4: Forces on the critical rocks in the simulation with inlet velocity of $1.4 \mathrm{~m} / \mathrm{s}$..............41

Table 4.5: Forces on the critical rocks in the simulation with inlet velocity of $1.5 \mathrm{~m} / \mathrm{s} \ldots \ldots \ldots \ldots \ldots . . . .41$

Table 4.6: Comparison of predicted failure upstream velocities from experiments and simulations.............................................................................................................. 44

Table 5.1: Forces on the critical rocks in the simulation with discharge of 30,100 cfs (100 year

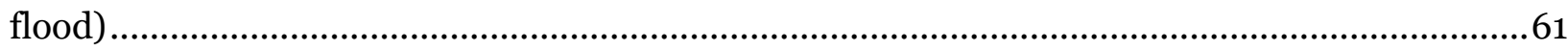

Table 5.2: Forces on the critical rocks in the simulation with average inlet velocity $12.2 \mathrm{ft} / \mathrm{s}(1.1$ *

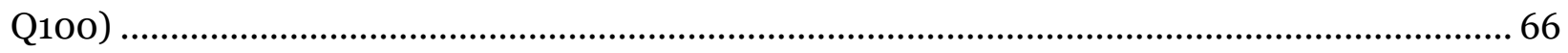

Table 5.3: Forces on the critical rocks in the simulation with average inlet velocity $12.2 \mathrm{ft} / \mathrm{s}$ (1.1 * Q100) 


\section{Introduction}

\subsection{Problem statement}

Local scour at bridge piers is a potential safety hazard of major concern to transportation agencies. If it is determined that scour at bridge piers can adversely affect the stability of a bridge, scour countermeasures to protect the pier should be considered. Riprap is one of many countermeasures alternatives to prevent scour and to secure piers from failure. One of locations where the riprap was used to mitigate the worsening conditions near piers is the Middle Fork Feather River Bridge. It is located on State Route 89 in Plumas County, California near the town of Blairsden and Graeagle. The bridge was built in 1955 and the river channel was realigned at the time to straighten the flow upstream of the bridge. In 1988 during high winter flows the bridge has avulsed from the channel realignment project constructed at the time the bridge was built and has moved toward its historic streambed and flow path [1]. As a result, the flow now enters the bridge at a strong angle and causes excessive backwater and deep scour at one of the piers (Pier3). The aerial view of the current condition together with a sketch of the historic channel alignment are shown in Figure 1.1. As an aftermath, the bridge was determined to be scour critical based on the resulting combination of vertical contraction scour and the local pier scour. To mitigate this scour critical condition, a rock mattress consisting of 1 Ton rock over filter fabric was placed around one of the piers in 2011. However, the design of the rock mattress did not consider the increase in flow velocity under the structure caused by the flow separation below the superstructure from the vertical contraction of the flow [1]. This has triggered more advanced analysis of which part is presented in this report.

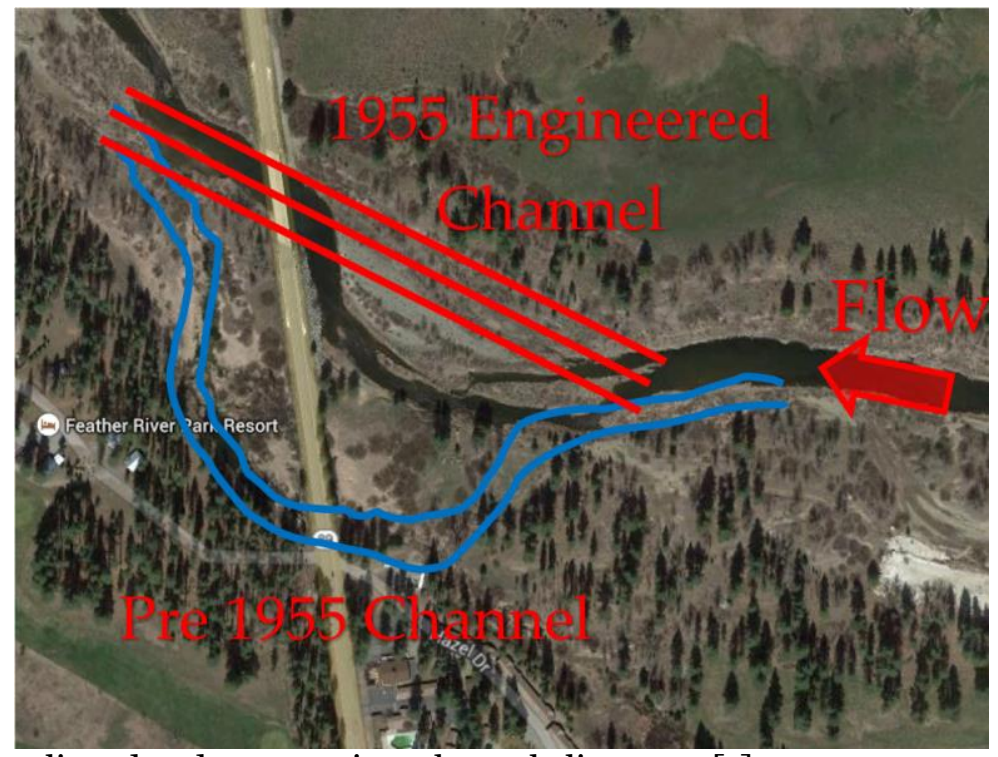

Figure 1.1: Historic, realigned and current river channel alignment [1]

The sizing of riprap in scour countermeasure design procedures is based mostly on limited field observations and scaled laboratory tests under ideal, controlled conditions. The size of riprap 
required for many field applications is too large for testing in any laboratory. As a consequence there is significant uncertainty in the formulas for sizing riprap. For example, as shown in Figure 1.2, there is a large spread in the design mean diameters of riprap, $\mathrm{D}_{50}$, from formulas that have been proposed between 1973 and 1999 for piers. Most of these yield larger values than the current HEC-23 formula [2], which is functionally equivalent to the 1936 Isbash formula with an added $K$ factor applied to the mean velocity that is meant to account for the higher instantaneous velocities around different pier shapes that are a function of the turbulence intensity.

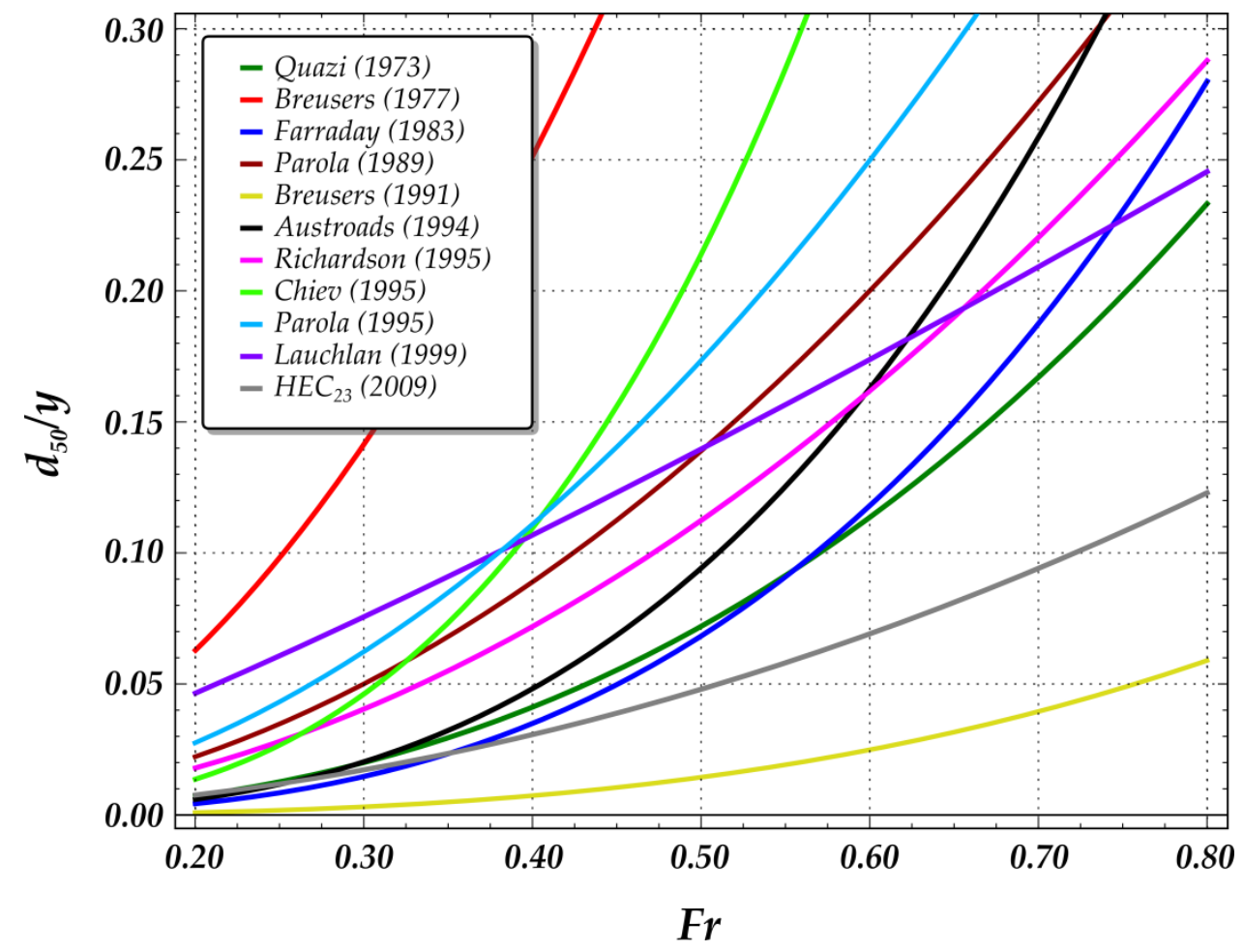

Figure 1.2 Comparison of riprap sizing curves at a rectangular pier based on [3]

Current design methodologies and scour evaluation procedures do not provide a clear means to analyze when the rocks might become displaced and, hence, further advanced computational mechanics techniques are required to assess the rock stability and, thereby, ascertain the scour vulnerability of the bridge.

From the computational mechanics point of view, the analysis of riprap stability can be considered a Fluid Structure Interaction (FSI) problem. FSI problems involve solving for the fluid flow force on a solid surface, the response of that solid to the load, and subsequently the change of the flow conditions caused by displacement of the solid. Historically computational fluid dynamics (CFD) software used for solving fluid flows and computational structural mechanics (CSM) software used for solving the deformations and stresses in solid bodies were developed independently. In recent years a number of CFD and CSM software vendors have been developing the capabilities needed to solve FSI problems within an integrated piece of software. In many cases these vendors are recognized leaders in the field of either CFD or CSM, but not both, and integrated FSI software, if available, is not yet mature and well tested by industry on large scale 
models. Until industry proven FSI solvers are available, coupling highly robust and reliable CFD and CSM software through the development of data exchange and concurrent control coupling procedures appears to be the best approach to solving complex, engineering FSI problems. TRACC has licenses, a user base, and in house expertise in the use of the software for Siemens's STAR-CCM+ CFD software and LSTC's LS-DYNA CSM software. For this reason, these software packages were chosen to develop coupling procedures for the detailed analysis of the onset of motion of riprap rocks. Siemens (formerly CD-adapco) was contacted regarding the feasibility of coupling and provided a list of the basic steps needed in the data exchange to couple STAR-CCM+ with CSM software.

This report describes the development of a coupling process between CFD and CSM software that provides the capability to carry out riprap stability analysis and to support the riprap failure risk assessment analysis. The report also contains the description of verification and validation efforts of the procedure that provide a necessary credibility of the analysis. Towards its end, the report describes the application of the developed coupling procedure to the analysis of the problematic riprap installation at the Middle Fork Feather River Bridge.

\subsection{Objectives}

NCHRP report 568 [3] lists four major failure modes for riprap revetments including (i) slope failure resulting in a slide, (ii) riprap particle erosion due to undersized riprap, (iii) erosion beneath the armoring layers of riprap, and (iv) erosion of the toe or key of the revetment leading to a slide failure. In the method developed here, detailed computation of riprap rock motion using FSI techniques can be applied to analysis of failure modes (i), (ii), and (iv). A scour model capable of handling scour beneath a riprap revetment would be required to analyze failure mode (iii).

Tracking incipient motion of riprap rocks in a real life application faces several problems. The most important one of them is the complexity of the geometry of the bed surrounding the affected pier. The riprap installation usually has hundreds of rocks placed in a semi-organized manner in several layers. Representing all the rocks in the model is impossible, however, sufficient engineering accuracy may be obtained with a reasonable approximation of the armor bed geometry.

The second problem pertains to the extent of the domain that has to be considered in the CFD model for proper analysis of flood conditions. The upstream boundary of the computational domain needs to be far enough away from the zone of interest so that the velocity profile can develop by the time the flow reaches the area of the bridge. In the varied bathymetry of a real river bed the variations constantly perturb the velocity profile. In most cases placing the upstream boundary about ten river hydraulic diameters upstream is sufficient. The downstream outlet boundary needs to be placed far enough downstream so that no recirculation zones created by flow obstructions, like piers, are crossing the outflow boundary. In that case the recirculation zone may pull fluid into the domain through the outlet boundary violating the boundary conditions and the computation normally diverges. The outlet boundary should also be far enough downstream so that conditions there do not influence the upstream zone of interest. Placing the outflow boundary about ten river hydraulic diameters downstream of the last obstruction is usually sufficient. 
STAR-CCM + is able to handle many millions of computational cells in a model addressing both of these issues. However, physically obtaining detailed bathymetry of the river bed is difficult and expensive, although exploration of current capabilities to map riverbed topology is briefly mentioned in this report as a reference to the research performed by Caltrans and TFHRC [4].

The third issue is a proper handling of the changes in the geometry of the CFD model. STAR-CCM+ is capable of solving flow problems in domains containing solid objects with complex, irregular geometry in relative motion along arbitrary paths through the fluid domain. Mesh motion and mesh morphing techniques were implemented in it for handling arbitrary motions of the objects. These features allow for deforming the computational mesh due to moving boundaries. In the process no new cells are created and the solution is mapped from the old mesh to the new deformed mesh. However, large displacements of the rocks, as well as, collisions between the riprap rocks, may cause the stretched cells to lose sufficient cell quality for accurate solution of the governing equations or on the other hand the algorithm may collapse some cells and cause negative volume cell termination of the computation. The feature that is still not present in STAR-CCM+ is an automatic interaction between morphing and remeshing of the domain in case cell quality becomes too poor. Also note that while STAR-CCM+ can handle some dynamic fluid body interaction problems for rigid body interactions with a fluid in motion and some capability to handle collisions between solid bodies, modeling of collisions is not yet sufficiently robust to handle the riprap onset of motion problem.

LS-DYNA software is a general purpose finite element program capable of simulating highly non-linear real world problems in structural mechanics including changing boundary conditions (such as contact forces between rocks that change over time), large displacements, large deformations, and non-linear material property relations. Recent updates to LS-DYNA included a release of a new CFD solver that is coupled to its structural solver. The feature of morphing and automatic remeshing interaction is already present in LS-DYNA, however, the CFD solver is still not capable of handling large domains required for modeling real flood cases, the meshing capabilities are very limited (tetrahedral elements only) and the physics models in LSDYNA are limited compared to the list of capabilities present in STAR-CCM+.

The presence of abovementioned issues makes it nearly impossible to solve the FSI problem for onset of riprap motion in either LS-DYNA or STAR-CCM+ alone. Thus, the primary objectives of this work were:

(1) Develop an efficient and automatic procedure for file based data exchange between STAR-CCM+ and LS-DYNA for the purpose of FSI modeling and in particular tracking the incipient motion of large riprap rocks in the river flow,

(2) Develop java macros internal to STAR-CCM+ to handle automatically update of the geometry and the mesh of the model based on rock motion data from LSDYNA,

(3) Prove the validity of the procedure through a simulation of lab controlled case, and

(4) Apply the developed coupling procedure to analyze the case of Middle Fork Feather River. 


\section{Coupling Methodologies for Solving FSI Problems}

\subsection{Governing Equations for the Fluid and Structural Domains}

While there has been an interest in solving FSI problems for decades, large computer clusters capable of solving them for full scale systems have only become widely available to engineers in the past decade. In addition, moving and morphing mesh capabilities in CFD software needed to solve FSI problems have only recently matured to the point where they can be reliably used. This maturity was necessary before the coupling of CFD and CSM software could be expected to be successful for large scale problems.

(a)

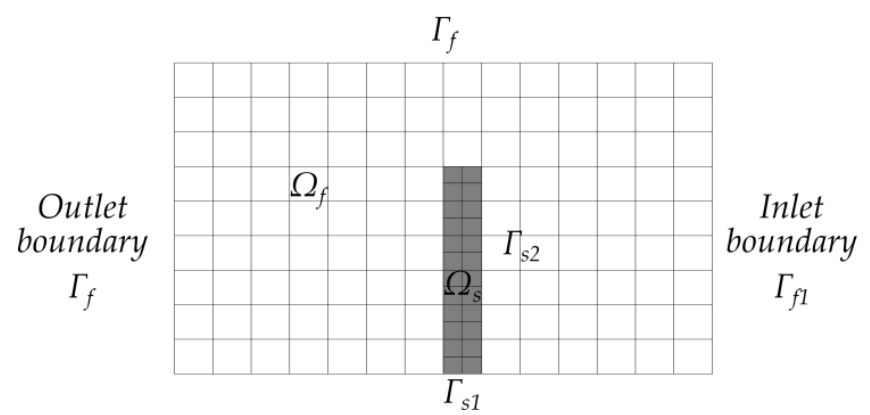

(b)

Mesh morphing

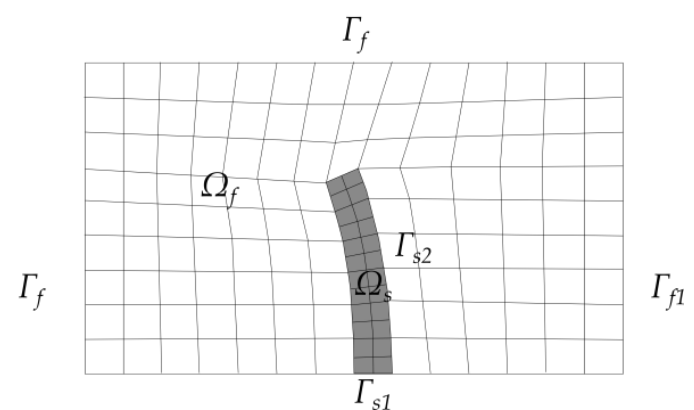

Figure 2.1: Initial stage of the FSI problem and an intermediate stage with mesh morphing

Figure 2.1a presents a schematic of a discretized computational domain with fluid occupying space $\Omega_{\mathrm{f}}$ and solid body occupying space $\Omega_{\mathrm{s}}$. It is a standard approach these days to use the Reynolds averaged Navier-Stokes (RANS) equations for Newtonian incompressible fluids with a $k-\varepsilon$ turbulence model to solve for the flow field and pressure distribution on the boundaries. The conservation of momentum and mass equations represented by RANS equations are (5):

$$
\begin{gathered}
\rho\left(\frac{\partial u_{i}}{\partial t}+\left(u_{j}-v_{j}\right) \frac{\partial u_{i}}{\partial x_{j}}\right)=-\frac{\partial p}{\partial x_{i}}+\frac{\partial}{\partial x_{i}}\left(\mu_{e f f} \frac{\partial u_{i}}{\partial x_{j}}\right)+\rho g_{i} \quad \text { in } \quad \Omega_{f} \\
\frac{\partial\left(u_{i}-v_{i}\right)}{\partial x_{i}}=0 \quad \text { in } \quad \Omega_{f}
\end{gathered}
$$

where $\rho$ is the fluid density, $u_{i}$ is the Reynolds averaged velocity in the i-direction, $v_{j}$ is the mesh velocity in the j-direction, $p$ is the pressure, $\mu_{\text {eff }}=\mu+\mu_{t}$ is the effective viscosity, $\mu_{t}=\rho C_{\mu} k^{2} / \varepsilon$ is the turbulent viscosity, and $g_{i}$ is the i-direction component of the gravity vector. The standard equations for turbulent kinetic energy, $k$, and dissipation rate, $\varepsilon$, are given by: 


$$
\begin{gathered}
\rho\left(\frac{\partial k}{\partial t}+\left(u_{j}-v_{j}\right) \frac{\partial k}{\partial x_{j}}\right)=-\frac{\partial p}{\partial x_{i}}+\frac{\partial}{\partial x_{i}}\left(\left(\mu+\frac{\mu_{t}}{\sigma_{k}}\right) \frac{\partial k}{\partial x_{j}}\right)-\rho \varepsilon+\mu_{t} S^{2} \quad \text { in } \quad \Omega_{f} \\
\rho\left(\frac{\partial \varepsilon}{\partial t}+\left(u_{j}-v_{j}\right) \frac{\partial \varepsilon}{\partial x_{j}}\right)=-\frac{\partial p}{\partial x_{i}}+\frac{\partial}{\partial x_{i}}\left(\left(\mu+\frac{\mu_{t}}{\sigma_{\varepsilon}}\right) \frac{\partial \varepsilon}{\partial x_{j}}\right)+C_{1 \varepsilon} \frac{\varepsilon}{k} \mu_{t} S^{2}-C_{2 \varepsilon} \rho \frac{\varepsilon^{2}}{k} \quad \text { in } \quad \Omega_{f}
\end{gathered}
$$

where $S=\left(2 S_{i j} S_{i j}\right)^{1 / 2}$ is the norm of the mean strain rate tensor of the fluid, and the model constants are $C_{\mu}=0.09, \sigma_{k}=1.0, \sigma_{\varepsilon}=1.3, C_{1 \varepsilon}=1.44$, and $C_{2 \varepsilon}=1.92$. STAR-CCM+ contains options to select a wide variety of variations of RANS turbulence models. To better handle the geometry variations in the domain, the more general realizable $k-\varepsilon$ model with a blended wall function formulation to determine shear stress at solid boundaries was used for this work. Details can be found in the user guide [6]. The initial and boundary conditions for the fluid domain are:

$$
\begin{array}{cccc}
\left.\xi\right|_{0}=\xi^{0} & \text { in } & \Omega_{f}(0) \\
\xi=\xi_{b} & \text { on } & \Gamma_{f 1} \\
\frac{\partial \xi}{\partial n}=h_{b} & \text { on } & \Gamma_{f 2}
\end{array}
$$

where $\xi \in\left\{u_{x}, u_{y}, u_{z}, p, k, \varepsilon\right\}$ and $\xi_{b}$ and $h_{b}$ are known boundary values for Dirchlet, $\Gamma_{f 1}$, and Neuman, $\Gamma_{f 2}$, boundary conditions respectively. Information on setting consistent boundary values for inlet, outlet, pressure, wall, and symmetric boundaries is contained in the user guide [6]. The solid part of the domain $\Omega_{s}$ is governed by the following conservation equations (see references [7] and [8] for details):

$$
\begin{gathered}
\rho \frac{\partial u_{i}}{\partial t}=\frac{\partial \sigma_{i j}}{\partial x_{j}}+\rho g_{i} \quad \text { in } \quad \Omega_{s} \\
\sigma_{i j} n_{i}=t_{i} \quad \text { on } \quad \Gamma_{s 1} \\
\left.x_{i}\right|_{0}=x_{i}^{0} \quad \text { on } \quad \Gamma_{s 2}
\end{gathered}
$$




$$
\left(\sigma_{i j}^{+}-\sigma_{i j}^{-}\right) n_{i}=0 \quad \text { on } \quad \Gamma_{s 3}
$$

where $\sigma_{i j}$ are components of the stress tensor, $n_{i}$ are the components of the surface normal vector, $t_{i}$ are components of external surface forces, and equation 11 requires equality of contact forces at the interface of two solids in contact.

In most classical CFD problems the boundaries are fixed during the analysis, and the computational mesh does not change. Notable exceptions are turbo machinery and in-cylinder combustion simulation where special techniques were developed to handle the moving boundaries. In FSI problems the fluid boundaries may be part of a structure that will move or deform in response to surface and body forces that are determined as part of the solution of the problem. As the boundary motion is calculated, the computational mesh in the fluid domain has to be updated either by a morphing procedure or a complete domain remesh process. The coupling conditions on the interface between the fluid and solid domains of an FSI problem are:

$$
\begin{array}{ccc}
u_{f}=u_{s} \quad \text { on } \quad \Gamma_{f}=\Gamma_{s} \\
n \cdot \sigma_{f}=n \cdot \sigma_{s} \quad \text { on } \quad \Gamma_{f}=\Gamma_{s}
\end{array}
$$

where $\sigma_{f}$ and $\sigma_{s}$ are the fluid and solid side stress tensors respectively.

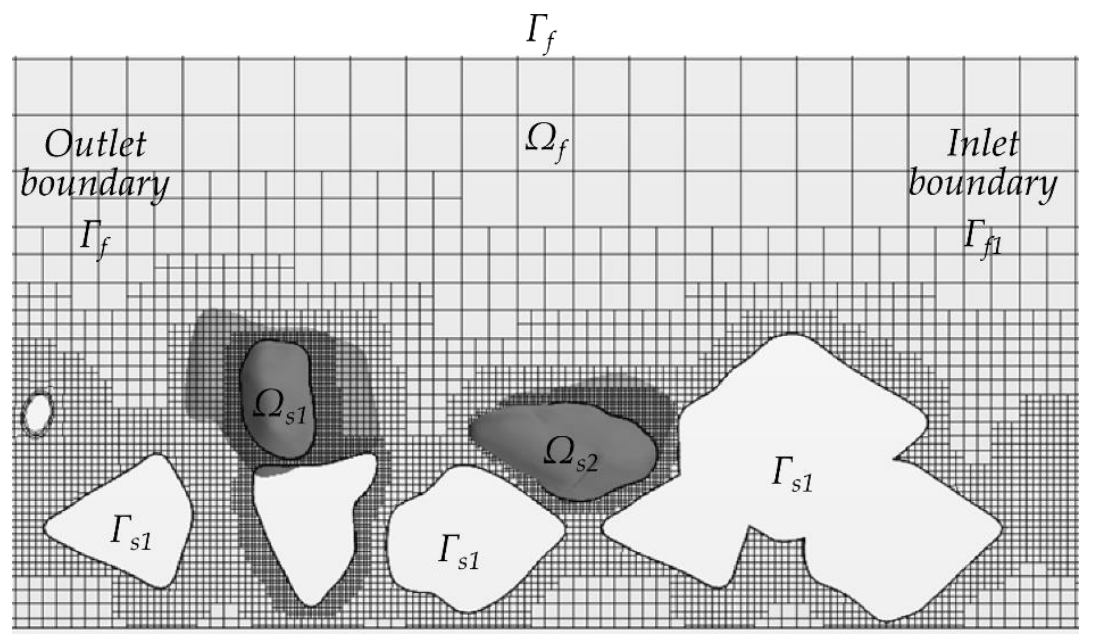

Figure 2.2: Definition of the domains for analysis of riprap stability as an FSI problem

The CFD solution of the fluid flow equations yields the detailed distribution of fluid stress on solid surfaces (left hand side of equation 13). This stress distribution is passed to the CSM software to solve for the response of the solid bodies, and that solution yields the displacement rate (velocity) distribution of the solid surfaces (right hand side of equation 12). In general, the surface velocity distribution may include both deformation and rigid body motion. In the analysis 
of riprap rock motion, it includes only rigid body motion. The motion computed by the CSM software is passed to the CFD software as a boundary condition that is a function of time.

\subsection{Methodologies of Coupling Codes}

In general there are two groups of coupling solutions for FSI problems: monolithic and partitioned [9]. The monolithic approach involves solving the coupled set of equations for the fluid and structural domain as a single problem. Although this approach may seem the most natural one, it can be more difficult to adjust solver parameters to obtain a converged solution than with the partitioned approach. Additionally, complex geometries and large displacements pose another challenge that is hard to overcome using this method. The use of robust CFD and CSM software from vendors who specialize in those areas naturally leads to using a partitioned approach where the equations are solved iteratively one domain at a time and coupling conditions are set via file based data exchange from the solution of the other domain.

Depending on the magnitude of the influence of interface boundary condition changes computed in either the solid or fluid domain on the other domain one-way or two-way coupling may be needed to solve the problem. If, for example, displacements of the structure due to fluid forces are small enough so that they do not substantially influence the fluid flow, then one way coupling from the fluid domain to the structural domain can be used. In this case the pressure distribution on the structure is not affected much by its motion, and therefore flow equations need to be solved only once to obtain the load from the flow on the structure. In riprap FSI analysis the motion of a rock results in an evolving position and orientation that substantially changes the pressure distribution over the surface of the rock, requiring two-way coupling for this problem.

Two way coupling can be either weak or strong. Weak coupling is presented in Figure 2.3a. In a weakly (or loosely) coupled step $n$, the solution in the fluid region, including the pressure and shear stress distribution, is found on the movable riprap wall the boundaries at the start of a time step obtained from the structural solver from the previous step $n-1$. This distribution is subsequently passed to the structural solver, which will yield a solution giving the solid boundary displacement and velocity that is passed to the CFD solver for use in time step $n+1$. The structural solver may require smaller time steps than the fluid solver if effects like contact, such as a rock colliding with surrounding rocks on the bed, are taken into account. Thus the fluid solver time step may be different than structural solver time step, however, the sum of time steps computed on both the structural and fluid domains between data exchange must be equal and that sum establishes the coupling time step. The number of steps and length of time steps is determined by the complexity of the physics modeled in each solver. The selection of the leading part in that scheme, CSM or CFD, is made by the user. Due to the fact that there are no inner iterations in that coupling step, this method is also known as the explicit type of coupling. 
(a)

\section{Loose FSI coupling}

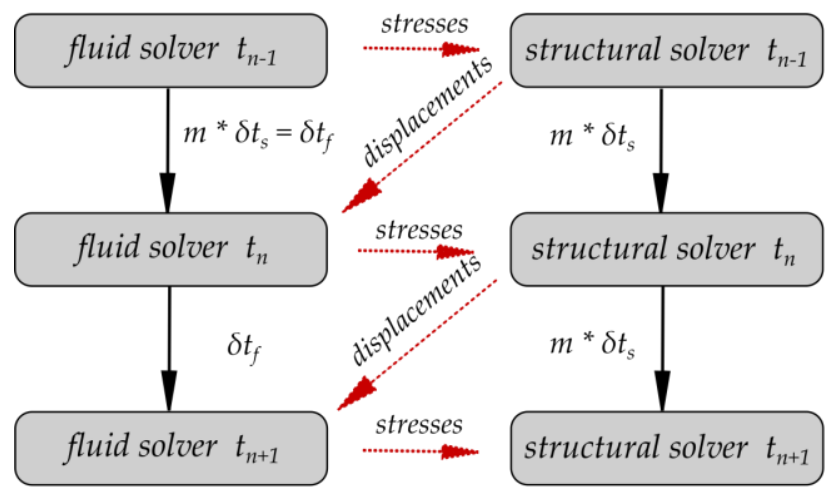

Figure 2.3: Loose and strong FSI coupling schemes (b)

\section{Strong FSI coupling}

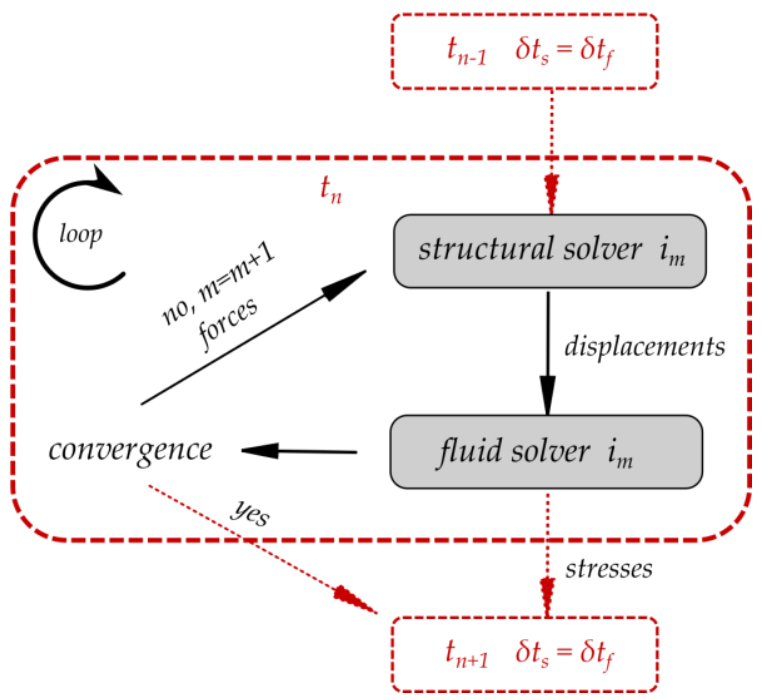

Figure $2.3 \mathrm{~b}$ presents a schematic of the strong coupling procedure. In this scheme the two problems are also numerically decoupled. However, within each time step $n$, the CFD and CSM solvers exchange interface conditions computed by the separate solvers during the iteration to converge the time step. An updated location of the structure's boundary in the structural step is fed to the CFD model and a new pressure field is found. Instead of progressing to the next step, the updated pressures are used one more time to find a different state of the structure within the same time step in the inner iteration $m+1$. Usually these new positions are averaged together with the positions obtained in the previous inner iteration $m$ to improve stability of the method. Due to the presence of the inner iterations in that scheme it is also called the implicit coupling method. It usually leads to more accurate results but for the linear increase of the computational cost with the number of inner iterations. For problems where oscillatory type of motion is not the primary response of the structure, good results can be obtained with the use of explicit coupling scheme with an appropriately adjusted coupling time step.

\subsection{Workflow of the File Based Data Exchange with Explicit Coupling}

The currently implemented explicit coupling involves two separate solvers: STAR-CCM+ [6] performing CFD calculations and LS-DYNA [7], [10] performing structural analysis. Both of these software packages have some FSI capabilities. However, LS-DYNA does not yet have sufficiently robust fluid flow solvers, flexible meshers and the wide range of flow physics models available in STAR-CCM+. On the other hand STAR-CCM+ just recently implemented the contact capabilities for rigid bodies, but the algorithm isn't capable enough to handle efficiently multiple rocks colliding with each other, additionally the small time step usually required by the contact algorithms would make the simulation prohibitively long. However, the authors believe that this situation is going to change in the future, expanding the area of application of STAR-CCM+ to a broader range of FSI problems. Currently a variety of models for particle-particle and particle- 
wall interactions exist in STAR-CCM+, but those are sub-grid models of particles not represented in the mesh. For the purpose of analyzing the onset of motion of riprap rocks, modeling the effects of contact forces between objects represented in the mesh is an essential feature. For these reasons the analysis is split into two sub problems. STAR-CCM+ calculates the flow field and the pressure distribution on rocks, while LS-DYNA calculates the motion of rocks due to the stresses exerted by the fluid on the rock surface and the effects of contact forces. A new position of a rock after the coupling step is subsequently imported into STAR-CCM+ as a basis for the next time step calculation. Because the rocks are treated as rigid bodies, and their behavior is far from regular harmonic motion, an explicit coupling procedure is sufficient to obtain first order accuracy in the solution of the rock motion. The small time step required in LS-DYNA to handle body interactions and in STAR-CCM + to keep the mesh morphing stable was assumed to be sufficient to compute the onset of rock motion and the trajectory to adequate engineering accuracy.

Figure 2.4 presents the workflow of the procedure to analyze incipient motion of riprap for a given arrangement and flow velocity. The procedure is executed in a LINUX environment with a control program written in the Python language. The program starts execution of needed components of LS-DYNA and STAR-CCM+, including the solvers and meshing software, and LSPREPOST (LS-DYNA pre- and post-processing software). It also translates output files into a neutral NASTRAN format recognized by both software packages.

The analysis procedure begins with initialization runs in both solvers started manually. The LS-DYNA run provides the initial position of the rocks under gravity loading in a drop simulation. This position is used as a basis for CFD domain geometry. The CFD model is run until pseudo steady state conditions are achieved with all rocks stationary. Subsequently the Python program is started with the scheme in which STAR-CCM+ simulation leads the coupling step. Execution of the CFD part is quite complex and requires an internal Java macro to run within STAR-CCM+ to import rock displacements and map them from the CSM mesh to the CFD mesh and vice versa. It is almost always the case that the resolution between the fluid and structural grids is different, especially when two separate solvers are handling the fluid and solid domains. STAR-CCM+ provides accurate data mappers for non-conforming meshes [6]. This mapping has to be performed at each time step as the underlying mesh deforms. The effect of mesh morphing as a consequence of body motion is presented in Figure 2.5. The displacements of the body are distributed throughout the morphed fluid domain to maintain cell quality. No cells are added or removed in the morphing process and their neighbor relationships are preserved so the mesh topology remains constant. The Arbitrary Lagrangian-Eulerian (ALE) algorithm is invoked to solve transport equations resulting from the moving underlying mesh [6, 10]. It allows for retaining the exact shape of the interface between the solid and the fluid.

Most of the effort in developing the FSI coupling went into resolving moving and morphing mesh problems that arise when rock motion collapses the space between a rock and another solid surface and problems that arose in the mapping and data exchange between the CFD and CSM software. The resolution of these problems yielded coupling software for data mapping, data exchange, and automated mesh morphing failure recovery that makes it possible to carry out the analysis without frequent intervention of the user. 


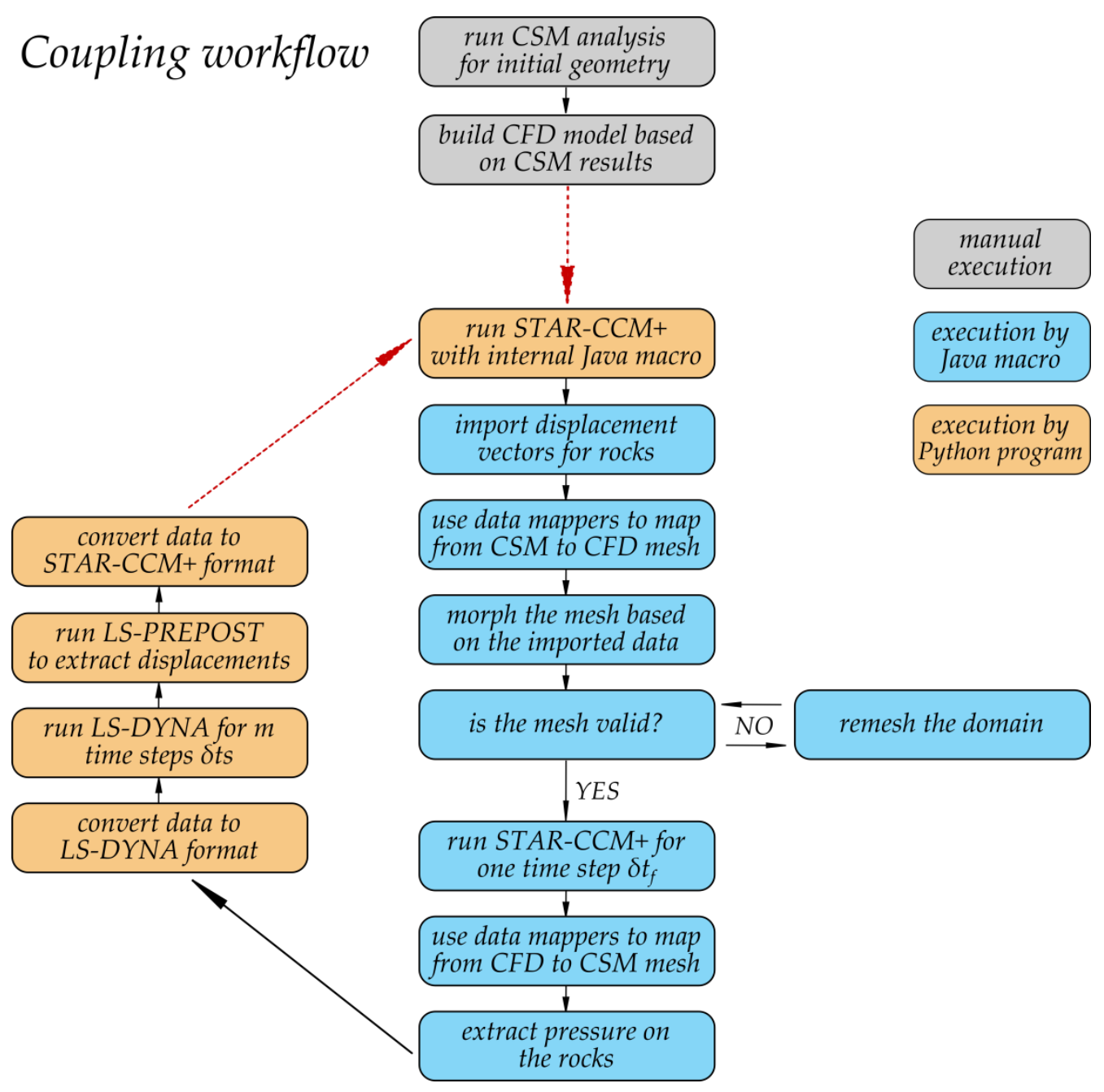

Figure 2.4: Current implementation of coupling workflow between STAR-CCM+ and LS-DYNA

The mesh morpher uses a sophisticated algorithm that yields a high quality mesh in the whole computational domain based on the initial mesh and the displacement of the boundaries. However, in cases where the displacement of a rock becomes large or it comes in contact with a solid boundary, the cells may become too distorted and of such poor quality that the solver either diverges or encounters a floating point exception condition. In such cases the Java macro executes a computationally demanding remeshing of the whole domain. Cross section of remeshed domain after failure of the mesh morpher is shown in Figure 2.6. The time step of calculations is selected such that full domain remeshing is avoided in the initial steps. The functioning of automated procedures is demonstrated in computation of water flow at increasing velocities until rock motion into the downstream occurs. A riverbed geometry with no obstructions and a geometry with an abutment corner are analyzed to illustrate the method in chapter 2.5.

Once a CFD time step $\delta t_{f}$ is converged, the pressure and shear stresses on movable rock surfaces are mapped from the CFD mesh on the rocks to the CSM mesh and exported to file. The Java macro as well as the STAR-CCM+ solver are stopped. Next an LS-DYNA simulation is 
performed for $m$ shorter time steps, $\delta t_{s}$, such that $m \delta t_{s}=\delta t_{f}$, the length of the coupling step. The resulting displacement vectors from LS-DYNA are extracted and translated to NASTRAN format so that they can be imported into STAR-CCM+ for the next step. After this, the whole loop is repeated until the termination time.

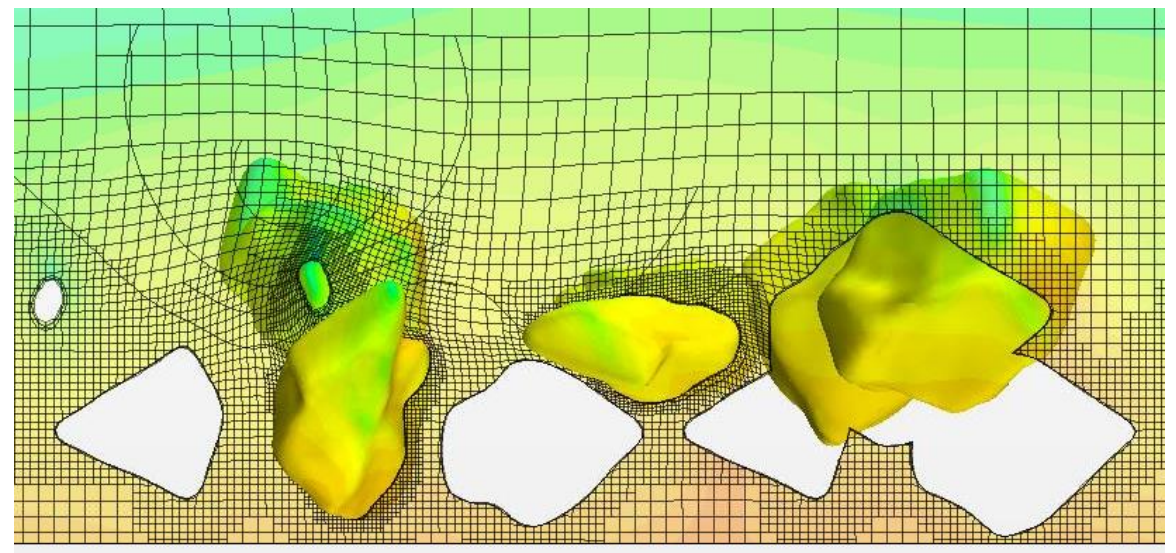

Figure 2.5: Mesh distortion as a result of morphing

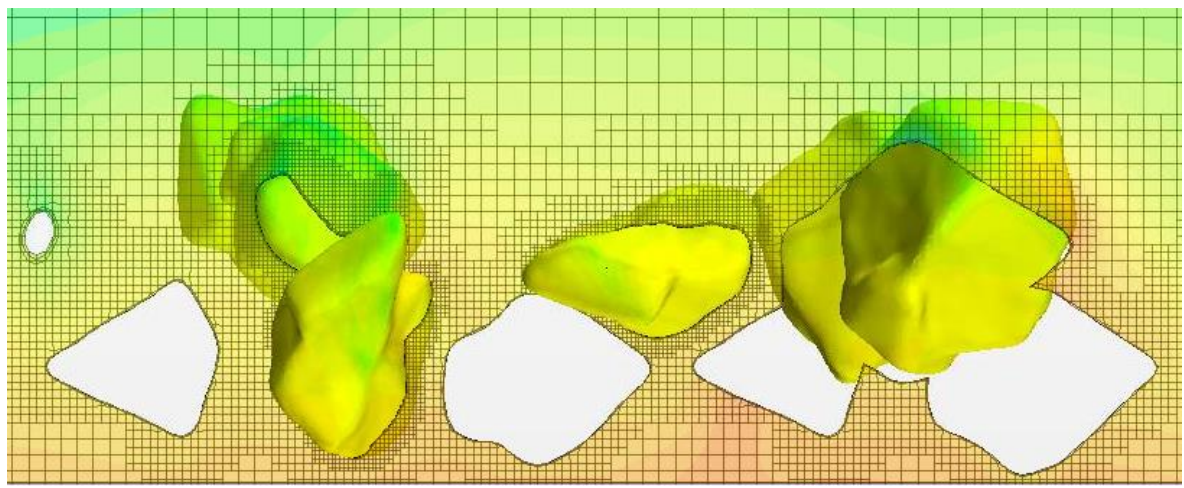

Figure 2.6: Remeshed domain after failure of the mesh morpher

The initial proof-of-concept analysis used the geometry presented in Figure 2.7. It consisted of only four rocks where only the top rock was free to move. The motion of three others was constrained in the CSM simulation. The coupling procedure worked very well in the first several steps. The volume mesh in STAR-CCM+ was morphed each time a new position of the moving rock was imported. However, initially undetectable, after several remeshing cycles the shape of the moving rock started to degenerate. During the remeshing stage a standard approach was to extract current surface mesh in STAR-CCM+ and build the new volume mesh based on that extracted surface. It turned out that the interpolation and discretization process introduced an error forcing the fluid volume to expand slightly beyond the boundary on irregular surfaces. As a consequence, the moving rock shrunk in size through repeated remeshing operations. Figure 2.7 shows stages of the rock motion due to the water flow over it and presents the described problem. The shape degeneration starts to be noticeable already in the middle row of the snapshots. It was concluded that the only workaround for this problem was to preserve the rock's irregular shape by wrapping the rock mesh with a large number of curves called feature curves. The meshing algorithms treat features differently from general vertices defining the position of a surface. For 
feature curves, the meshing algorithm tries to maintain the geometry of the curve within a much smaller tolerance than that of the surface in general.
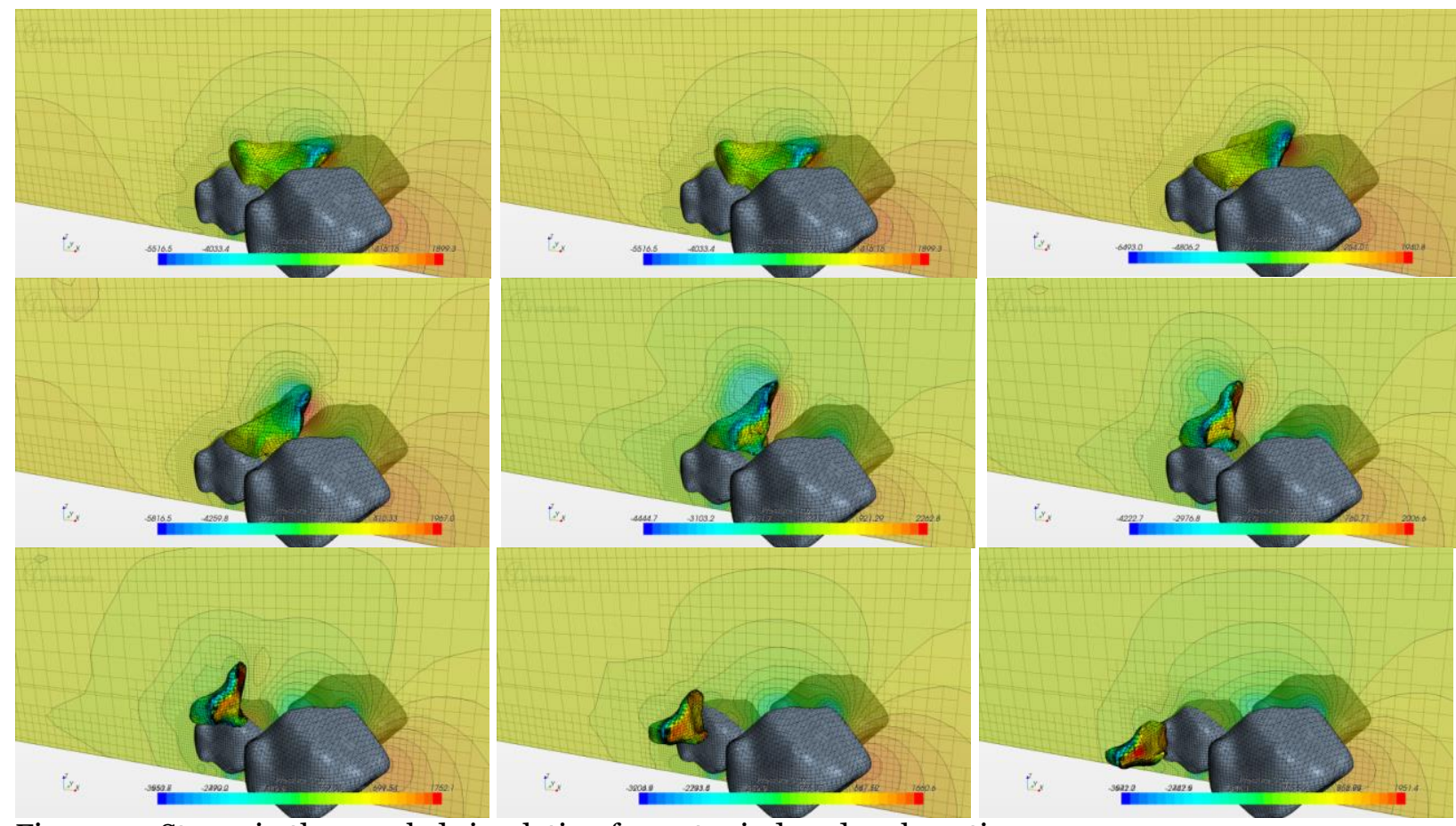

Figure 2.7 Stages in the coupled simulation for water induced rock motion

Figure 2.8 shows the finely meshed surface of a rock with dense feature curves on it. This issue was brought up here as it not only caused the slowdown of the progress in the current project but was also experienced on several other occasions when working on other projects and in the work of others.

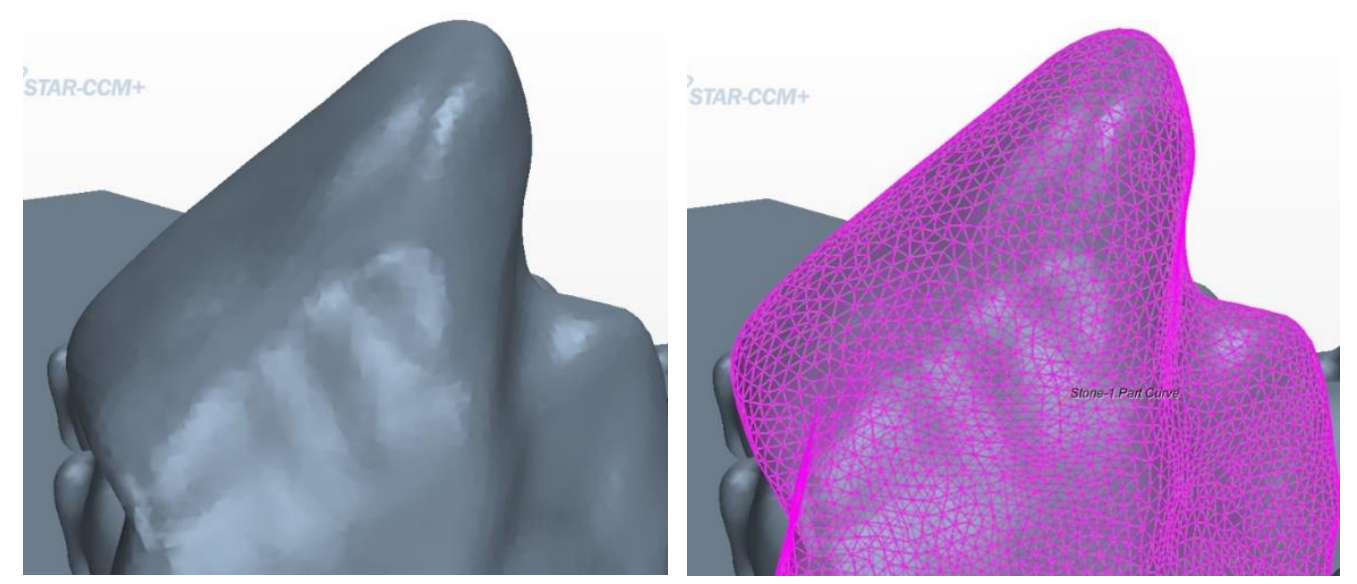

Figure 2.8: A rock surface and feature curves created on all edges of the mesh 


\subsection{Challenges, Limitations and Alternative Solutions of Current Implementation}

Using commercial software, as opposed to research software, has many advantages, the primary one being a large industrial user base engaged in using it successfully to solve problems on a daily basis, and consequently computed results are very reliable within the accuracy of the physics models used. Vendors responding to the needs of industry also implement a wide array of physics models, methods to solve a variety of moving mesh problems, and advanced solver algorithms. They normally provide a means for users to add new physics models in the form of user defined subroutines or functions. As such, they provide an excellent foundation for building models to solve new problems, or analyze classic problems using advanced analysis techniques. One disadvantage of using commercial software is that the users do not normally have direct access to the source code and therefore cannot make even minor modifications outside of the means provided by the vendor that might be needed for a new model development. That confines the user to the set of models and tools developed solely by the code owner that can be used when solving an engineering problem. The current implementation of coupling between STAR-CCM+ and LS-DYNA allows for capturing the main effects of rock motion, however, when domain remeshing occurs some of the information about the motion of a rock from step $n-1$ to step $n$ is lost, and the grid fluxes are not included in the momentum equations immediately after remeshing. As a result, the reduction in drag due to acceleration of a rock is not fully accounted for, and rock motion with large displacements will occur at a slightly lower velocity difference between the rock and mean flow. The error in this case is conservative in the sense that it would lead to slightly oversizing the riprap for a particular application. At the onset of motion the displacements and velocities of the rocks are so small that this effect should be small.

The fact that only an explicit coupling was implemented has its advantages and disadvantages. The obvious advantage is the computational time. Since within each coupling time step there are no inner iterations and only one exchange of the data is performed per time step, this approach is significantly faster than the implicit approach. For flexible structures vibrating in the flow, this approach frequently diverges quickly and an implicit (strong) coupling is needed to stabilize the solution. For the rigid rocks this behavior wasn't noticed and it was assumed to be sufficient for the engineering accuracy sought in this work.

While the procedure was under the development, Siemens introduced a new approach to modeling the motion of the objects within the computational domain using so called overset meshes. This approach doesn't rely anymore on morphing and remeshing of the domain. Instead it encapsulates a moving object in its own small domain that shares the space with the background global domain. During the computation the background mesh is carved out in the place where the local mesh is and new internal boundaries are built between the local and the global mesh. The results are interpolated from the global mesh to the local mesh on these boundaries and the local mesh is treated then as a part of the global model. This method is claimed to bring several advantages to the modeling of FSI problems: (i) reduction of the overall run time since there is no need for the most time consuming part - the remeshing, (ii) improvement of stability since only one mesh is used throughout the simulation, and (iii) improve accuracy of the results. 
As mentioned earlier the base procedure with mesh morphing and remeshing loses information on grid fluxes when the remesh is performed. The proposed approach with the moving overset mesh does not have this problem. An overlap of the subregions and the background mesh is computed at each time step of calculations and an interpolation interface between these regions are redefined accordingly. Active and inactive cells are found in the rock and background regions at the beginning of each time step. Additionally, motion of the overset regions is defined as rigid body motion based on the calculations performed in LS-DYNA (or internal FE solver of STAR-CCM+). For overset regions rigid body motion is specified by using just six components per time step. Previously, a displacement vector for each vertex on the rock boundary was defined separately.

The listed hypothetical gains made it worthwhile to test the new capability of the software. Figure 2.9 shows a cross section through the main domain and two rocks encapsulated in the local subdomains. The software allows for an overlap of multiple subdomains. Figure 2.10 shows a cross section through the main domain which was already carved out by the local mesh around the rocks. Overlapping meshes are noticeable on the edges of the subdomains. There is a requirement of at least four elements in that overlap region. There are obvious problems with this requirement near the boundaries and Siemens is constantly improving the special treatment of near boundary cells.

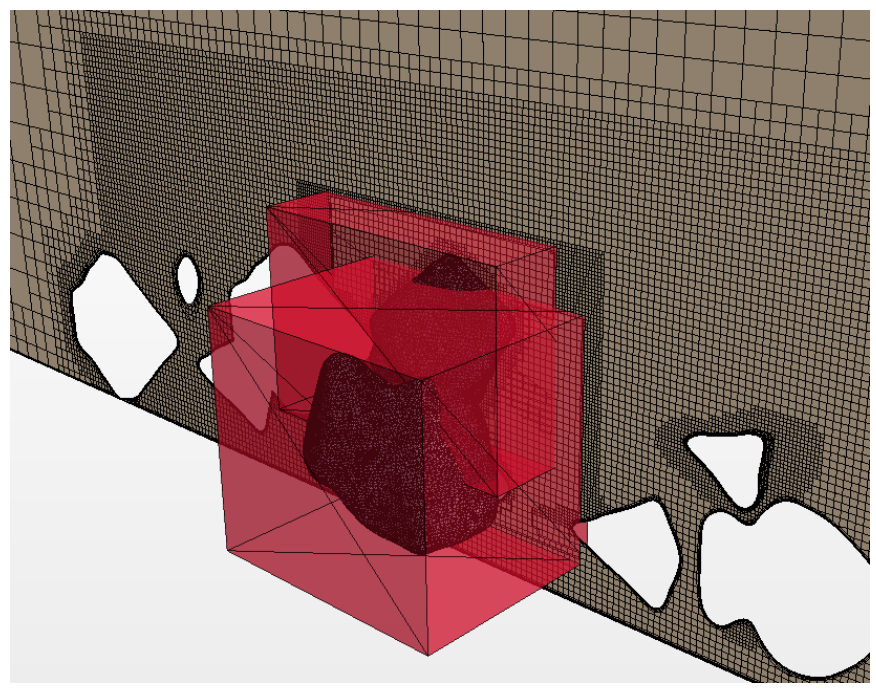

Figure 2.9: Definition of separate subregions for each rock 


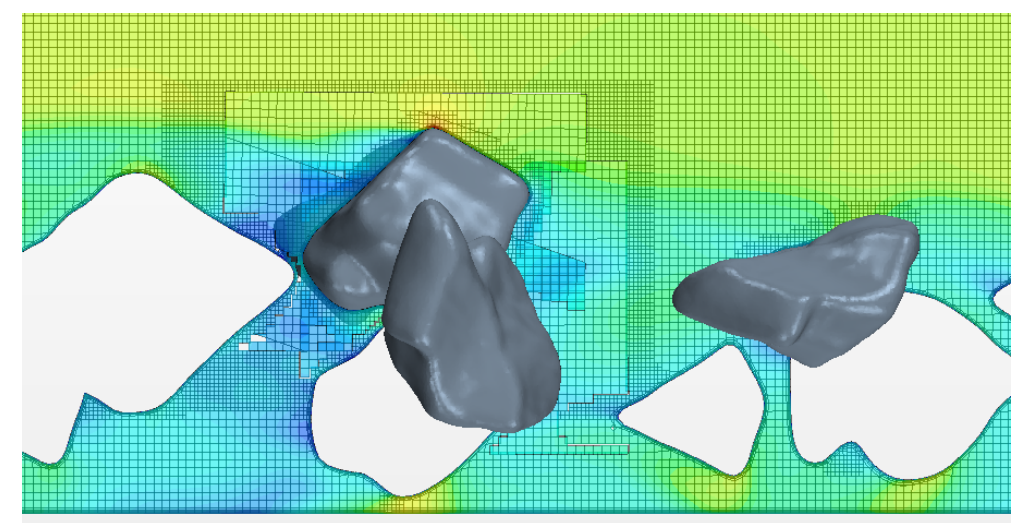

Figure 2.10: Overset meshes in the main domain and the subdomain with the moving rock

Figure 2.11 presents the implementation of the coupling workflow between STAR-CCM+ and LS-DYNA with overset mesh motion instead of mesh morphing and remeshing. In the initial implementation, the displacement of the rock was imported to STAR-CCM+ as a set of displacement vectors for all the vertices defined on the rock surface. Subsequently the mesh morphing took place and if the mesh was valid the CFD calculation was executed. In the case of invalid cells (poor quality cells) a remesh procedure was executed. In the coupling with overset meshes the displacement of the rock is defined as a rigid body motion with only three linear and three angular displacement vectors. The velocities are computed based on the defined time step of calculations.

The overset mesh procedure is more computationally efficient than the procedure based on mesh morphing and remeshing. The coupling iterations were executed faster than the previously described implementation. However, while the procedure with overset meshes solves some problems, it introduces new ones. When tracing the forces acting on a single rock during its motion, it was noticed that this approach generates unrealistically high forces on the rocks that do not converge to reasonable values within one time step. Figure 2.12 shows this behavior in one of the simulations. Once the coupling was turned on around iteration 510, the $\mathrm{Z}$ force increases significantly from about 20 to $250 \mathrm{~N}$. In the following time step it drops back to a value below 50 $\mathrm{N}$. However, in the next time step it decreases to about $-180 \mathrm{~N}$ and within another couple of time steps it goes back to positive value below $50 \mathrm{~N}$. These changes had no explanation in the changing flow patterns. Thus, this procedure was concluded to be unreliable at the current stage of development. 


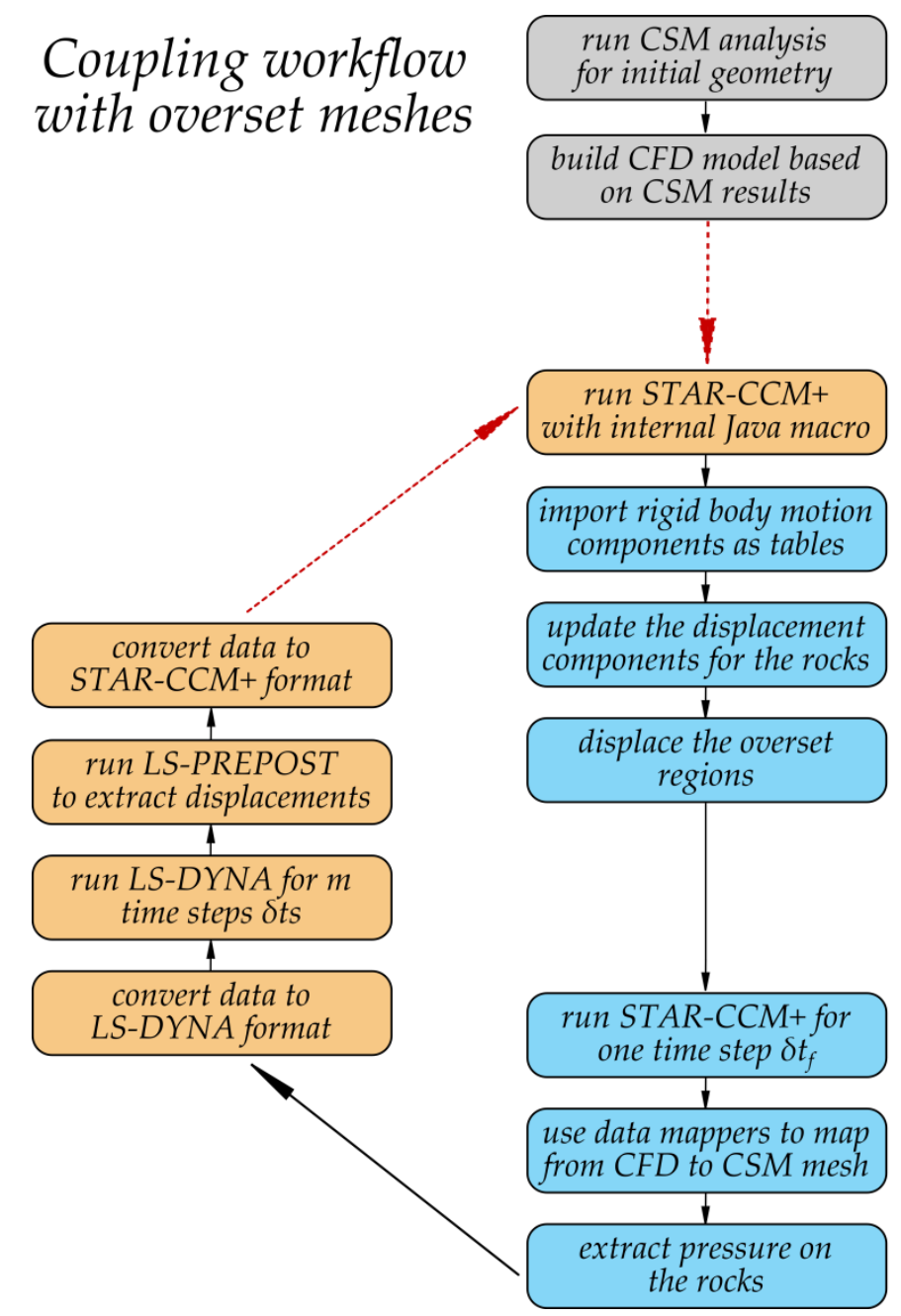

Figure 2.11: New implementation of coupling workflow between STAR-CCM+ and LS-DYNA with overset mesh

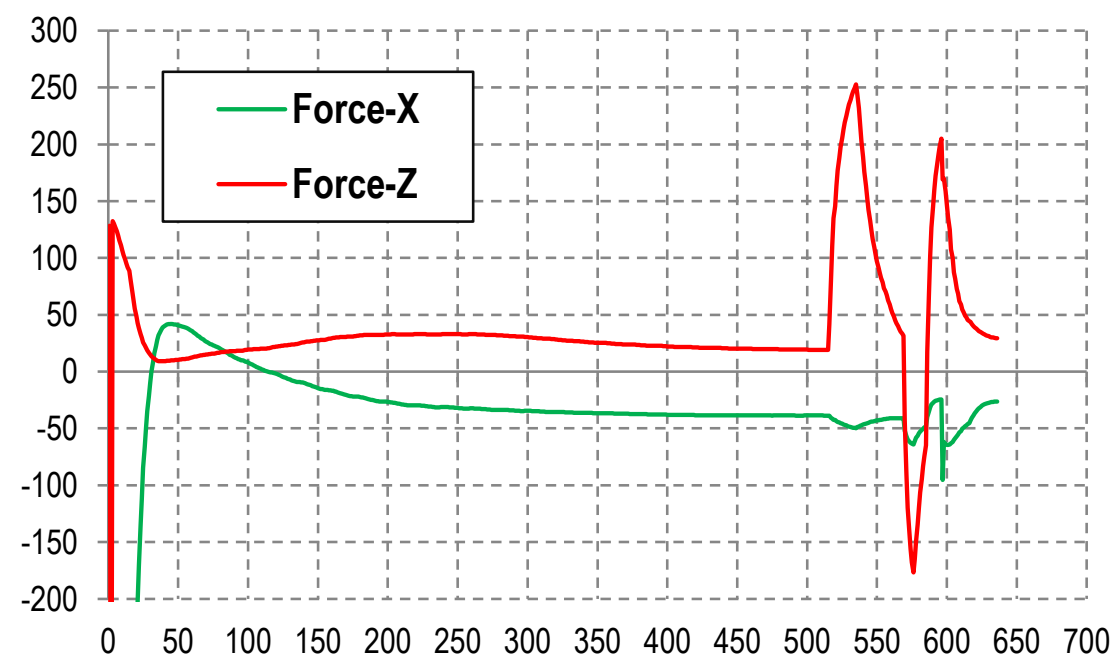

Figure 2.12: Forces in the coupling with overset mesh 
The overset mesh method has been under heavy development and improvements are expected in future releases of STAR-CCM+. Additionally, recent implementations of the overset mesh approach also allow for contact between moving objects and wall boundaries. Initial tests indicated that the implementation of contact algorithms in STAR-CCM+ is not robust yet. However, with the short development cycles of STAR-CCM+ it may be possible to simulate the entire FSI riprap problem in STAR-CCM+ only once these components and models work well together with the Finite Element solver internal to STAR-CCM+ in near future.

The procedure with morphing and remeshing seems to be conceptually most reliable at the moment. It appears to generate the most stable results which also are conservative, and for that reason, it was used to perform all the calculations reported in this document.

\subsection{Application of the Methodology to a Flat Bed Case}

The coupling procedures described in this report were developed primarily for the purpose of applying them to the analysis of incipient motion of rocks used in riprap around piers or abutments to prevent scour of the riverbed at these structures. Initially, however, a very simple flatbed geometry with only riprap rocks present was used as a development base until all of coupling problems were resolved. This simple case also allowed for comparison with Laursen's and Neill's equations for critical velocities [11].

Model development was started by processing the surface point cloud data from a $3 \mathrm{D}$ laser scan of a rock with $\sim 4 \mathrm{~mm}$ spacing between the points provided by researchers at the TurnerFairbank Highway Research Center [4]. MeshLab [5] software was used to generate a surface triangulation of the rock from the point cloud data (see Figure 2.13). The initial shape was subsequently modified by simple geometrical operations to create a set of rocks with similar shapes to populate the domain with rocks for testing. Several different rock shapes were used to create the configurations shown in Figure 2.14. Two layouts were considered in this analysis, layout 1, plain, Figure 2.14b, and layout 2, with abutment, Figure 2.14c. The grey rocks in the figures represent stationary rocks; these cannot move in the simulations as the FSI coupling was not activated for them. The three colored rocks in Figure 2.14b labeled rock 1, rock 2, and rock 3 can move and interact with other boundaries and among themselves through contact algorithms enabled in LS-DYNA. Rock 1 and rock 2 have exactly the same shape as the originally scanned rock in Figure 2.13. Rock 3 is bigger and more round than the other two but its longest internal measurement is the same, approximately $0.2 \mathrm{~m}$ (8 in). Rock 1 is placed flush with the other rocks in the bed. Rock 2 is placed quite deep under the top surface. Rock 3 is sticking out slightly above the bed. The shape of the rocks and their layout influences when they will be picked up and moved by the flow. Placing the rocks at different locations ensures that each of them will have a different critical velocity determined by the detailed geometry and flow field computed in the simulation. 

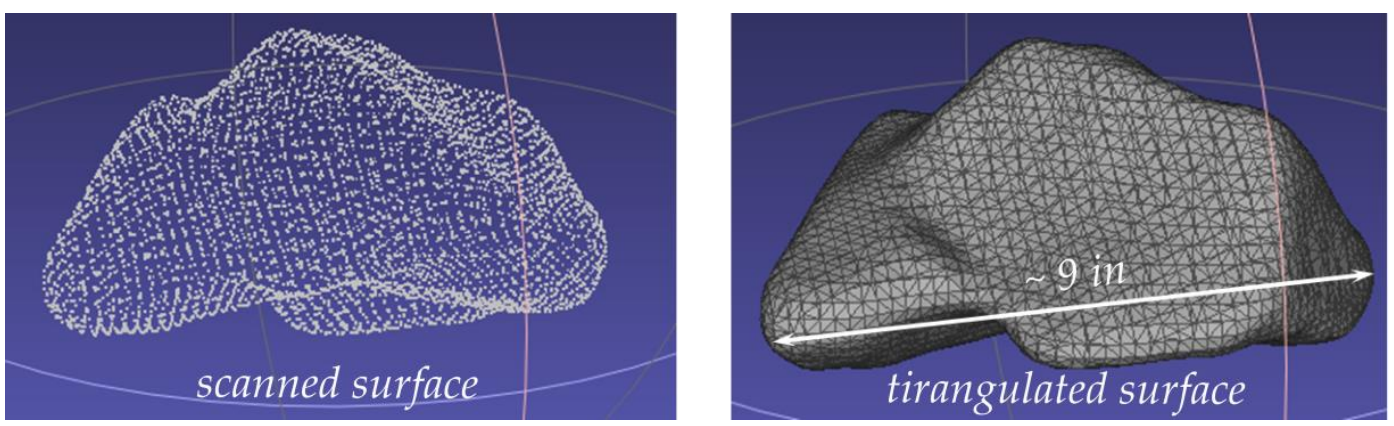

Figure 2.13: Point cloud of a scanned rock and its triangulated representation

A CFD domain was built with dimensions: $5.0 \mathrm{~m}$ long, $1.2 \mathrm{~m}$ wide, and $0.65 \mathrm{~m}$ high in the recessed area containing the rocks of the riprap layer. The height of the water from the top to the riprap bed top surface was approximately $0.35 \mathrm{~m}$. The size of the domain was designed to be small to conserve computational resources and provide fast turnaround times during development and testing of the coupling procedures. A single phase rigid lid model of the channel flow was also used to keep the model as simple and fast running as possible.

The right end of the domain was set as a velocity inlet. The initial water velocity in the domain and the velocity at the inlet was a variable parameter that was manually changed from 2.0 to $3.0 \mathrm{~m} / \mathrm{s}$ in $0.5 \mathrm{~m} / \mathrm{s}$ intervals. The sides of the domain were set as symmetry planes. The right side of the model was set as an outflow boundary. In layout 2, with the abutment, the abutment is a wall boundary and the approach flow boundary on the abutment side of the domain was also set as wall boundary. The opposite side is set as a symmetry plane. The domain was meshed using hexahedral cells with varying cell size from $0.004 \mathrm{~m}$ (0.16 in) around the rocks up to $0.06 \mathrm{~m}$ (2.4 in) away from the rocks and other boundaries. The total count of cells in the model varied between 1.5 million up to 2.0 million cells depending on the layout (plain or abutment) and the evolving position of rocks during the simulation. A k- $\varepsilon$ turbulence model with ReynoldsAveraged Navier Stokes equations was used to solve for the flow field. An implicit unsteady solver with time step of $0.01 \mathrm{sec}$ was used in the flow solver. That was also the coupling time step i.e. the rate at which data between the CFD and CSM solvers was exchanged.

In LS-DYNA the rocks were modeled as rigid bodies with a density of 2.9 metric tons $/ \mathrm{m}^{3}$ corresponding to granite. The mass of rock 1 and the rock 2 was $4.1 \mathrm{~kg}(9.0 \mathrm{lb})$ and the mass of the rock 3 was $7.1 \mathrm{~kg}(15.6 \mathrm{lb})$. Three contact definitions were incorporated in the model: (i) among the moving rocks, (ii) between the moving rocks and the stationary rocks and (iii) between the moving rocks and the boundaries. It is sometimes advantageous in LS-DYNA to wrap solid bodies with null, massless shell elements to have more flexibility in defining the contact properties. One of advantages of such an approach in this case is the use of a non-zero thickness layer around the rocks that prevents them from coming into a full contact in STAR-CCM+. Using this method prevents morphed cells in a near contact zone from being squeezed to near zero volume and causing flow acceleration to unrealistically high velocities in crevices. The time step of calculations in the LS-DYNA explicit solver was set to $4.5 \times 10^{-6} \mathrm{sec}$. Use of the explicit solver and such a small time step was needed to stabilize the contact forces between rocks colliding with other rocks or wall boundaries. A time step that is too large can cause excessive contact forces and abnormal 
behavior of the rocks. Once the STAR-CCM+ and LS-DYNA models were initialized, the coupling between them was activated.
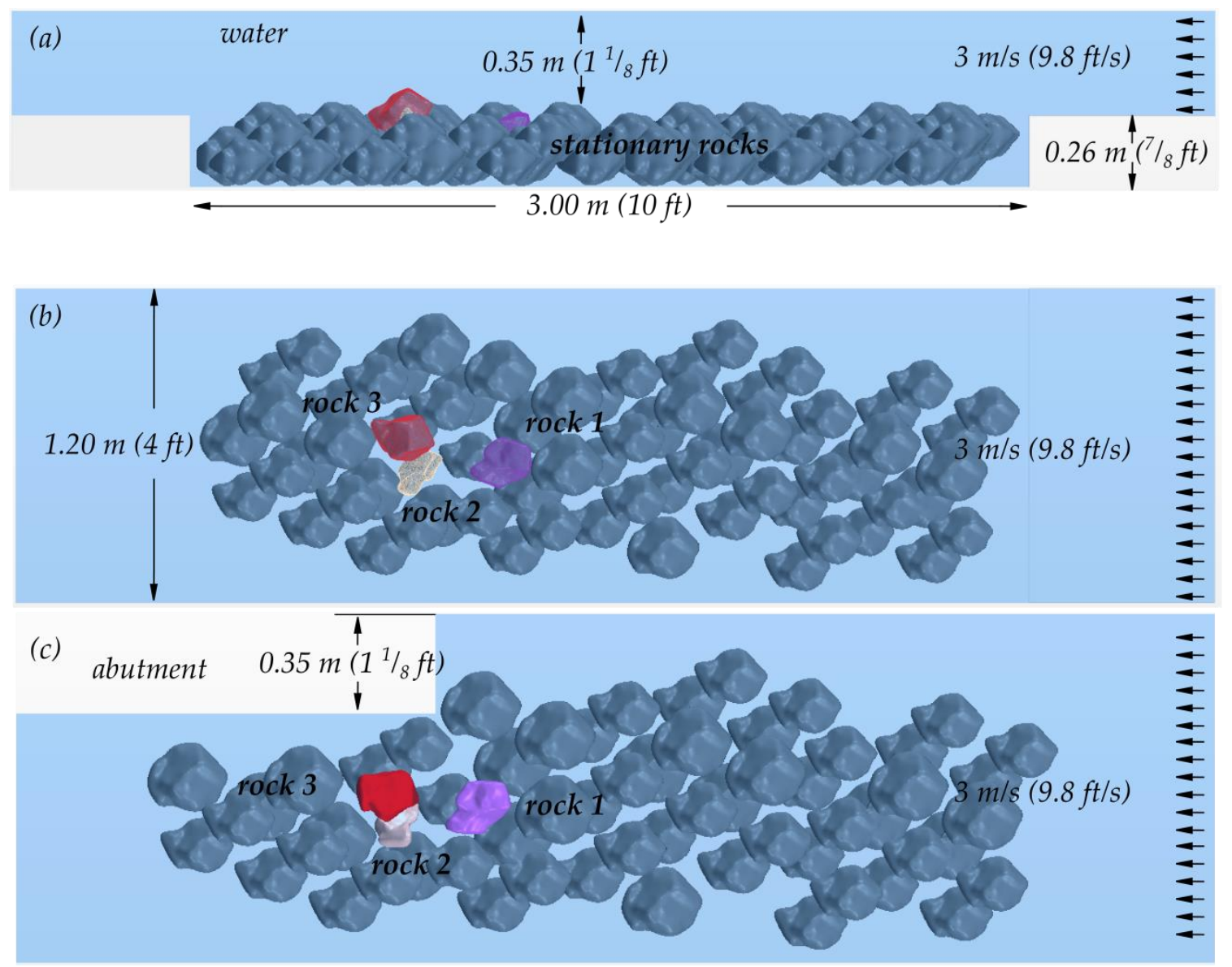

Figure 2.14: Geometry of the CFD model (a) layout 1, side view (b) layout 1, top view (c) layout 2, top view

Approximate values for critical velocity of the modeled rocks can be found by using Neill and Laursen equations [11]. Laursen's formula is given by equation 14:

$$
V_{C L}=K_{U} y_{2}^{1 / 6} D_{50}^{1 / 3}
$$

Where:

$K_{U}$ is 6.19 for SI units or 11.7 for US customary units,

$y_{2}$ is the equilibrium scour flow depth ( $\mathrm{m}$ or $\mathrm{ft}$ ),

$D_{50}$ is sediment size $(\mathrm{m}$ or $\mathrm{ft})$.

For larger sediment sizes, above $0.03 \mathrm{~m}$ ( $0.1 \mathrm{ft})$, Neill's formula for critical velocity is defined by equation 15 : 


$$
V_{C N}=11.5 K_{U} y_{2}^{1 / 6} D_{50}^{1 / 3}
$$

Where:

$K_{U}$ is 0.55217 for SI units or 1 for US customary units.

Taking $\mathrm{y}_{2}=0.35 \mathrm{~m}, \mathrm{D}_{50}=0.24 \mathrm{~m}$, the critical velocities can be estimated: $V_{C N}=3.31 \mathrm{~m} / \mathrm{s}$ $(10.8 \mathrm{ft} / \mathrm{s})$ and $V_{C L}=3.23 \mathrm{~m} / \mathrm{s}(10.6 \mathrm{ft} / \mathrm{s})$. Based on that information, simulations were run with an inlet velocity of $2.0 \mathrm{~m} / \mathrm{s}$ and increased by $0.5 \mathrm{~m} / \mathrm{s}$ until a large displacement of a rock was observed. The results for the detection of motion of the rocks in specific runs are listed in Table 2.1. For both layout 1 and 2, at an inlet velocity of $2.0 \mathrm{~m} / \mathrm{s}$ there was no significant motion of the rocks. For both layout 1 and 2 and an inlet velocity $2.5 \mathrm{~m} / \mathrm{s}$ a local motion of rock 3 (the largest and most exposed to the flow) was noticeable, but it was not moved out of its position. For layout 1 and an inlet velocity of $3.0 \mathrm{~m} / \mathrm{s}$, rock 3 was lifted out of position and moved into the downstream. Rock 2 also started to move locally. Snapshots from that simulation are shown in Figure 2.15. The fixed-in-place rocks were removed from view for easier viewing of the moving rocks. A semitransparent cross section through the domain showing the velocity distribution in the water is also shown in the figure. Snapshots of rocks from the simulation with layout 2 and inlet velocity of $3.0 \mathrm{~m} / \mathrm{s}$ are shown in Figure 2.16.

Table 2.1: Motion of the rocks in two analyzed cases

\begin{tabular}{|l|l|l|}
\hline $\begin{array}{c}\text { inlet } \\
\text { velocity }\end{array}$ & \multicolumn{1}{|c|}{ layout 1 } & \multicolumn{1}{c|}{ layout 2 } \\
\hline $\mathbf{2 . 0} \mathrm{m} / \mathbf{s}$ & no motion & no motion \\
\hline $\mathbf{2 . 5} \mathrm{m} / \mathbf{s}$ & local motion of rock 3 & local motion of rock 3 \\
\hline $\mathbf{3 . 0} \mathrm{m} / \mathbf{s}$ & motion of rock 3 and local motion of rock 2 & motion of rock 1 and 3 \\
\hline
\end{tabular}



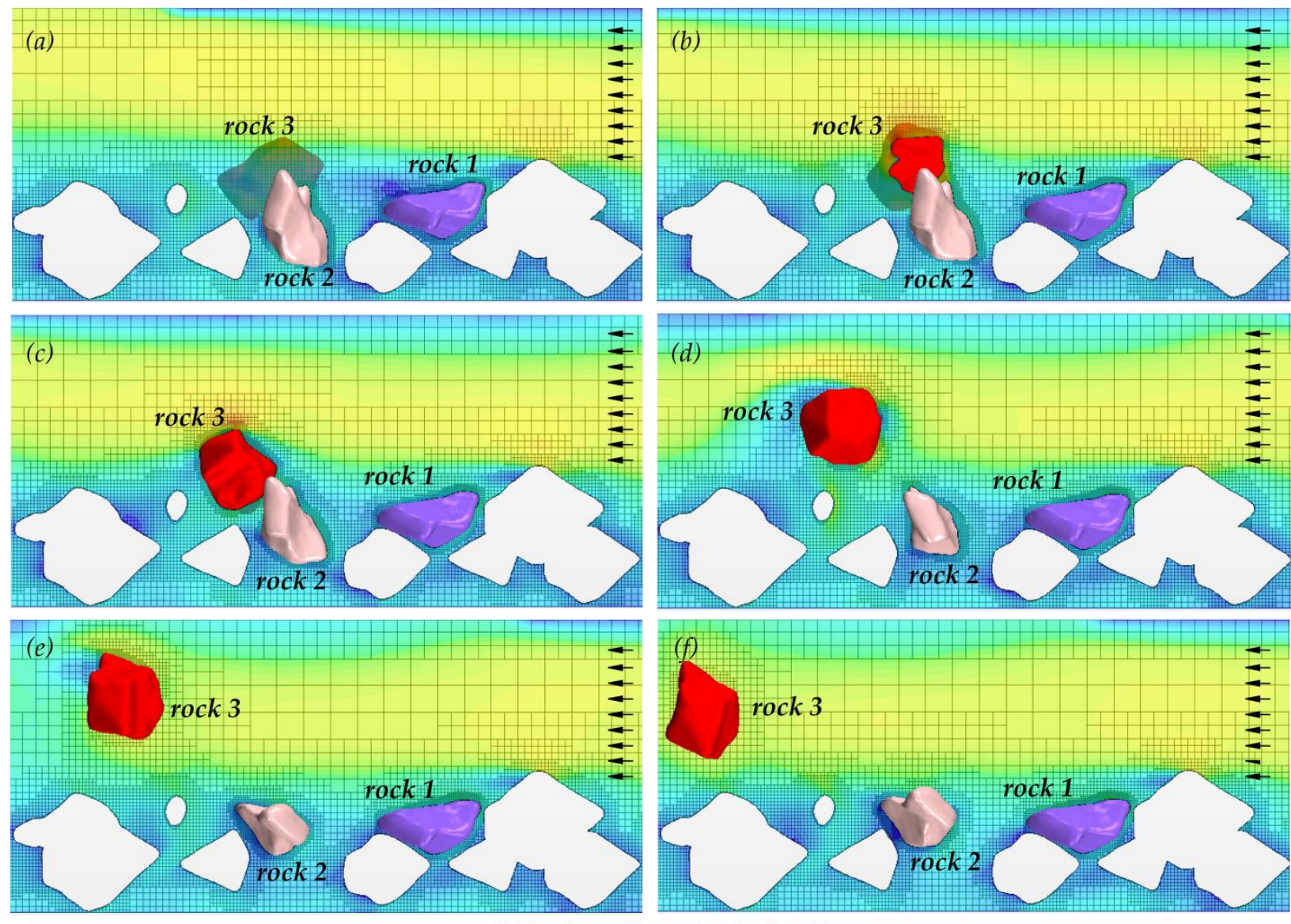

\subsection{0}

0.859

Velocity: Magnitude $(\mathrm{m} / \mathrm{s})$

1.72 2.58

3.44

4.30

Figure 2.15: Snapshots from simulation for layout 1 and inlet velocity of $3.0 \mathrm{~m} / \mathrm{s}$ 

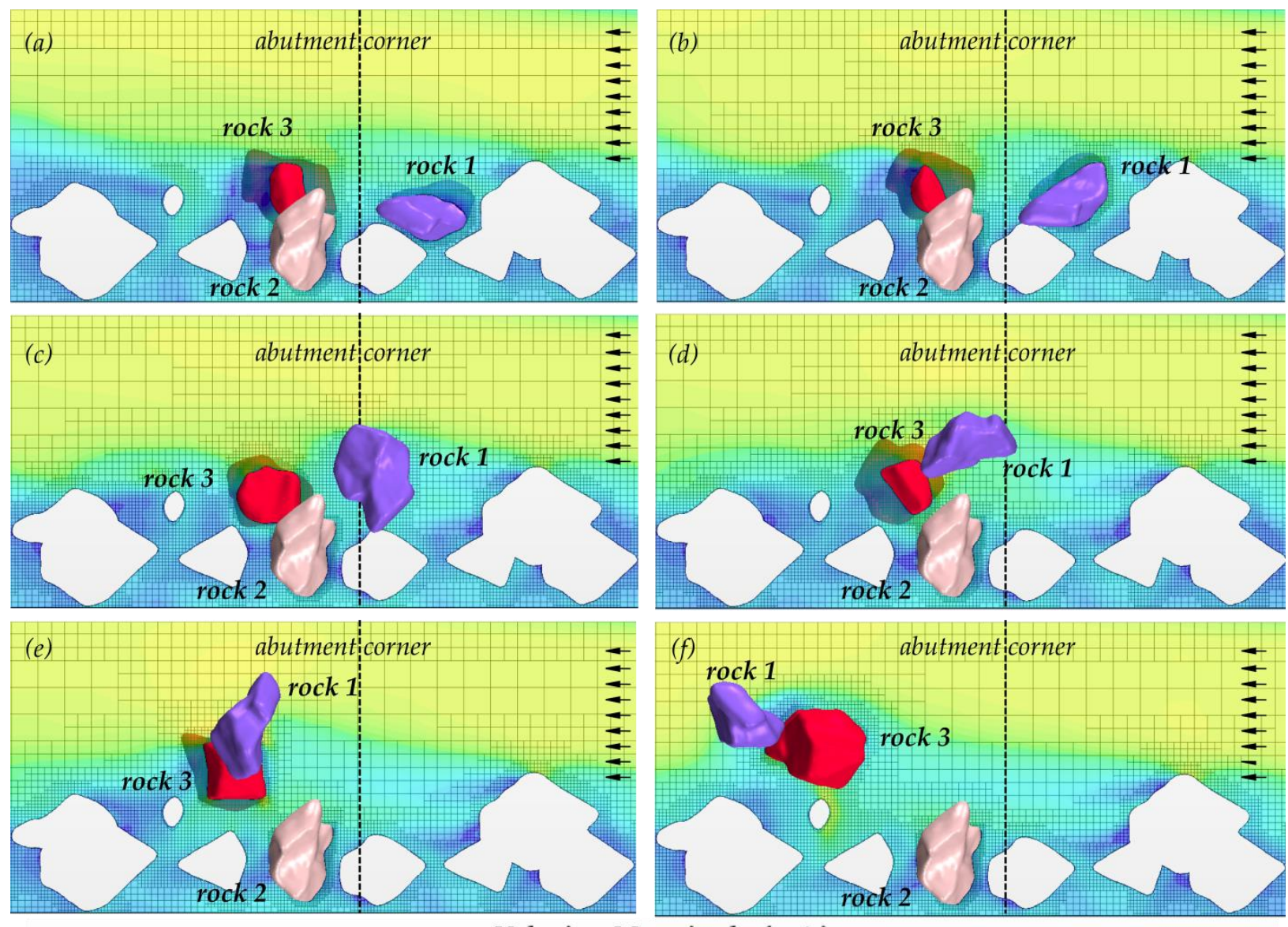

Velocity: Magnitude $(\mathrm{m} / \mathrm{s})$

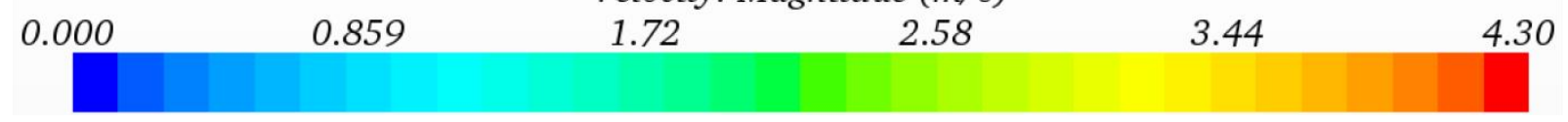

Figure 2.16: Snapshots from simulation for layout 2 and inlet velocity of $3.0 \mathrm{~m} / \mathrm{s}$ 


\section{Verification of the Coupling Mechanism through comparison with a STAR-CCM+/Abaqus Benchmark FSI Problems}

The STAR-CCM+ manual contains an FSI example solved in a coupled system of STAR$\mathrm{CCM}+$ and ABAQUS. STAR-CCM+ has a built in interface for this coupling and it allows for strong and weak coupling of the codes. For the verification purposes the same problem was also solved using developed here coupling procedure. The problem pertains to vibrations of a flexible rectangular plate standing in a $10 \mathrm{~m} / \mathrm{s}$ cross wind. The plate was constrained with its bottom edge perpendicular to the flow direction. The size of the modeled domain was respectively: a width of $0.29 \mathrm{~m}$, a height of $0.2 \mathrm{~m}$, a length of $0.9 \mathrm{~m}$. The plate was: $0.1 \mathrm{~m}$ high, $0.08 \mathrm{~m}$ wide, and 0.0025 $\mathrm{m}$ thick. A flexible material with elastic properties was used to model the plate material with the following material constants: Young's modulus 38.4 MPa, Poisson's ratio of o.3, and density equal to $4096 \mathrm{~kg} / \mathrm{m}^{3}$. The plate was located $0.4 \mathrm{~m}$ from the inlet (see Figure 3.1).

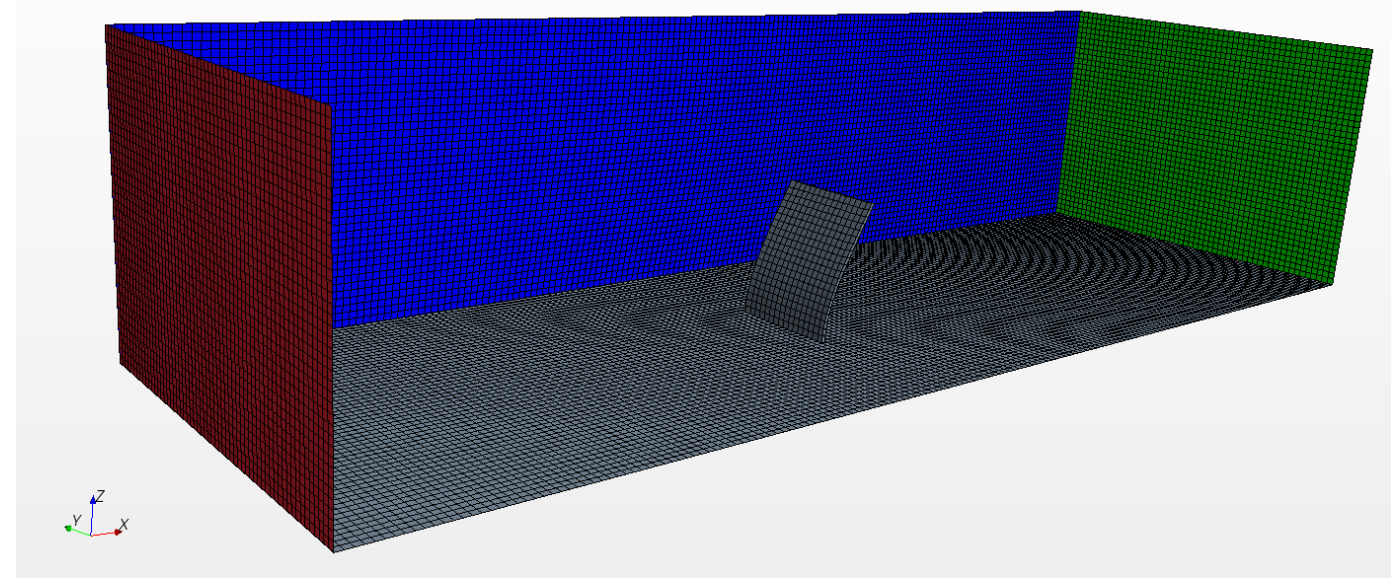

Figure 3.1: CFD domain and grid for analysis of flexible plate protruding into the flow setup for FSI analysis coupling with LS-DYNA

Figure 3.2 shows the mesh in proximity to the deformed plate for the coupling with ABAQUS and LS-DYNA. Siemens modeled the domain with polyhedral cells while here it was modeled with hexahedral cells. The overall model was built with around 300,000 cells. The configuration shown is captured at the state of the largest deflection of the plate. Figure 3.3 shows the displacement history of the two top corners of the plate. The maximum amplitude and the period of vibrations was predicted to be about the same magnitude by both coupling mechanisms. The local oscillations of corners of the plate behaved slightly differently. This was attributed to differences in coupling handling (explicit vs. implicit) and different theoretical formulation of the solid elements. 

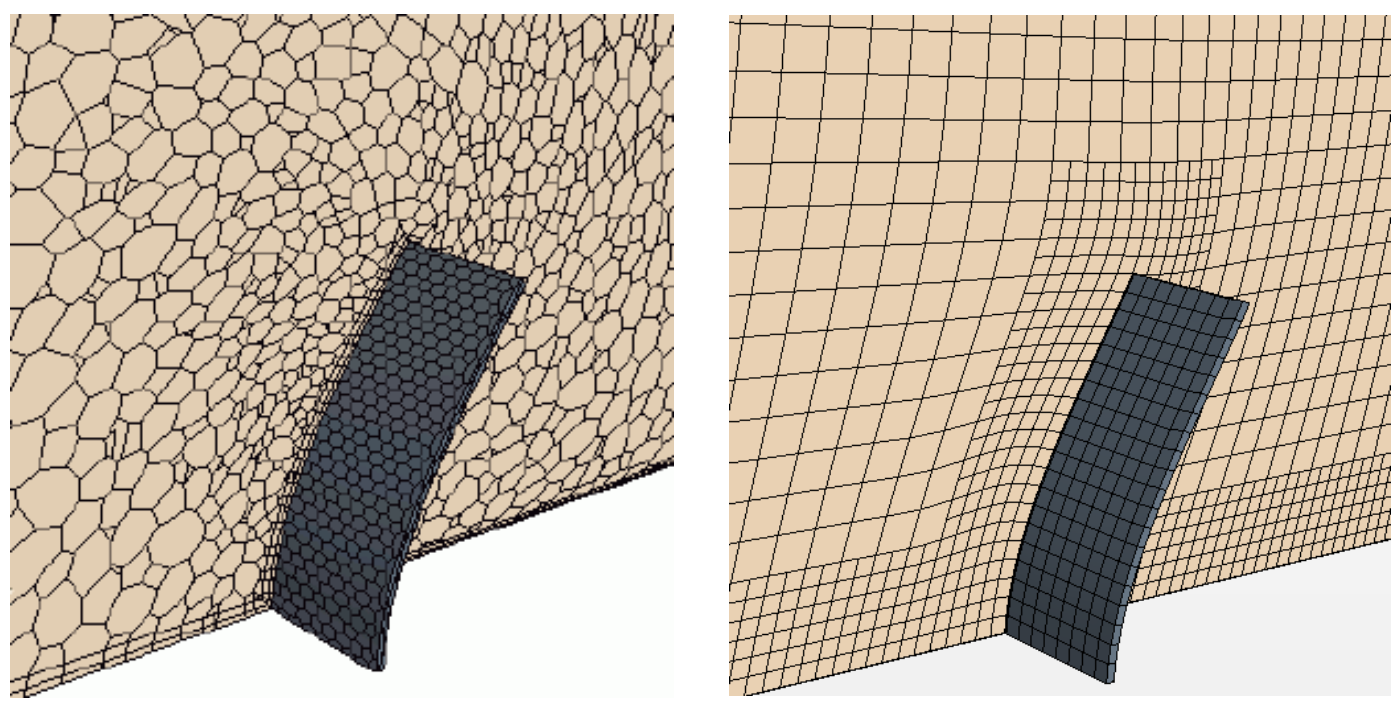

Figure 3.2: Morphed mesh at maximum plate deflection in (a) ABAQUS coupling (b) LS-DYNA coupling
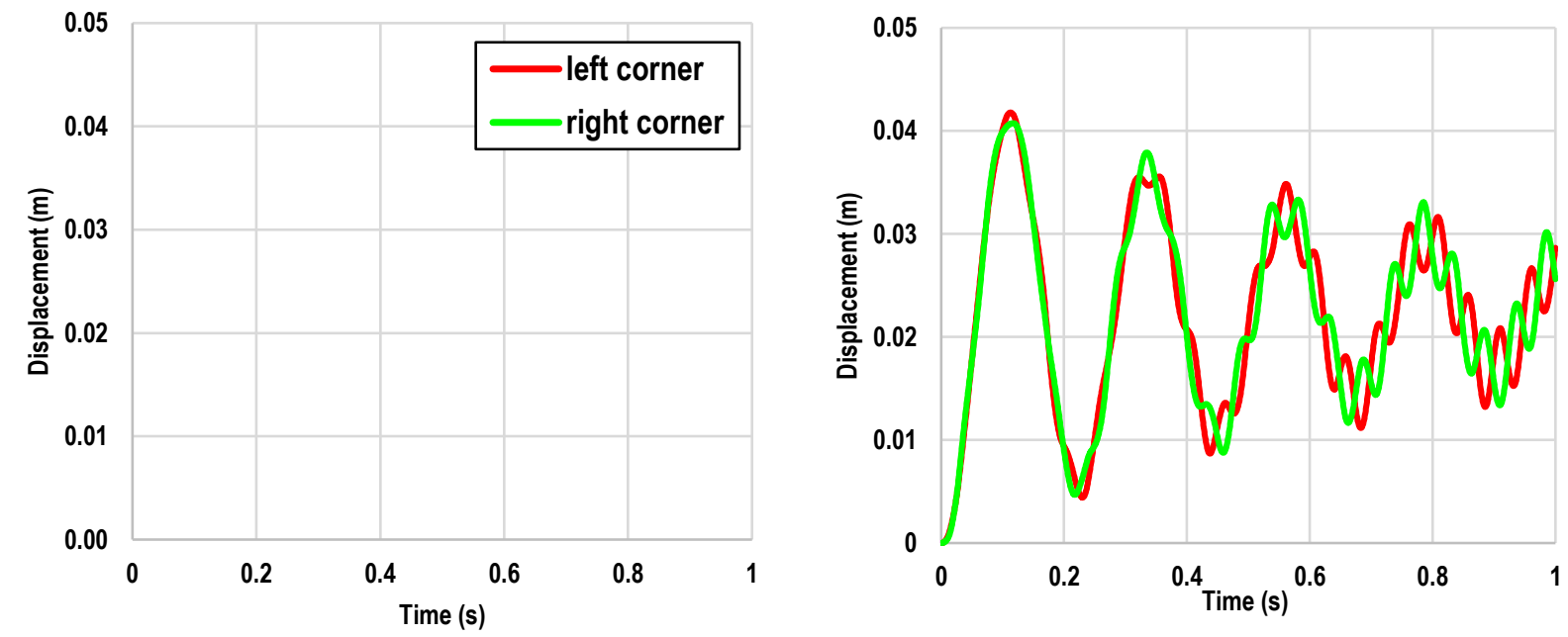

Figure 3.3: Time history of the plate deflection in (left) LS-DYNA - STAR-CCM+ coupling (right) Abaqus STAR-CCM+ coupling

Figure 3.4 shows velocity vector plot on the surface cutting the domain in half in the direction of air flow. Figure 3.5 presents the pressure distribution in that plane at the state of largest deformation of the plate. In the plot the morphed mesh is also displayed. Even with a coarse mesh the oscillations of the plate can be captured precisely. The mesh density and time interval between two coupling steps are dependent on the problem type and source of vibrations in the structure. 


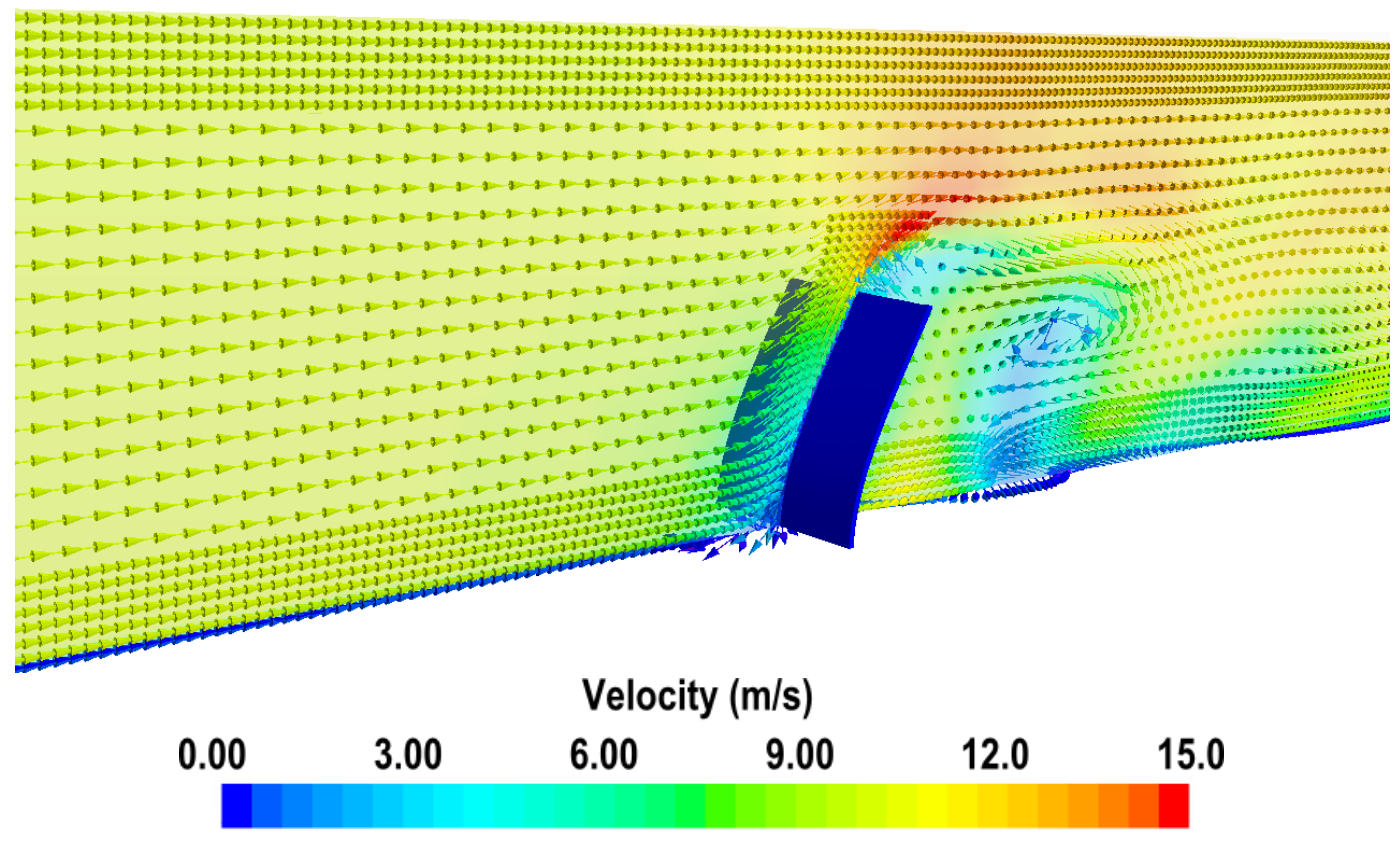

Figure 3.4: Velocity vector plot in the middle plane at maximum plate deflection

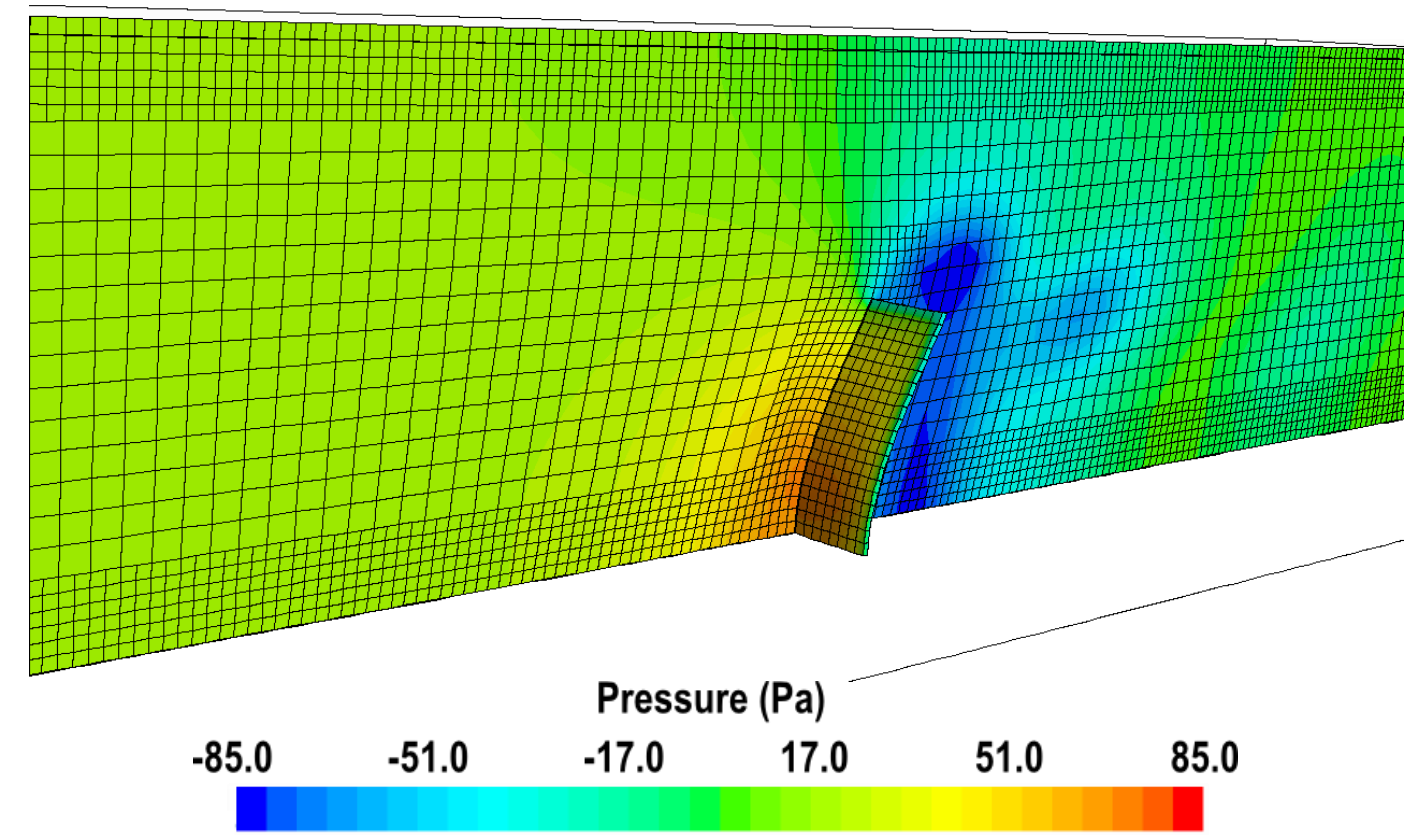

Figure 3.5: Pressure distribution in the middle plane at maximum plate deflection 


\section{Validation of the Methodology}

A validation procedure requires comparison of the simulated results with experimental ones. It is nearly impossible to perform a validation of this coupling procedure with full scale riprap performance studies as none can be closely monitored. To address this issue, TFHRC designed and conducted a lab experiment at scale 1:18. Subsequently a series of simulations of that experiment were performed for the full scale geometry. The results of the experiment were scaled using Reynolds number similarity and were used as a prediction of the numerical results. The following chapter describes briefly the experiment and the simulation efforts for validation purposes.

\subsection{Experimental Testing at TFHRC}

\subsubsection{Geometry of the Test Section}

The geometry consisted of two rectangular abutments installed on both sides of the channel. The test abutment was a geosynthetic reinforced soil (GRS) abutment while the other one was built of clear Plexiglas. Two layers of riprap $\mathrm{D}_{50}=17 \mathrm{~mm}$ were installed around both abutments following provisions of HEC23 D.G.14. The dimensions of the testing channel as well as the geometry of the abutments and the extent of the riprap are shown in Figure 4.1. In the parenthesis the full scale dimensions used in the simulation are shown. The riprap apron was installed flush with the non-erodible channel bed, meaning the average rock layer protrusion exposed to the flow was between 0.1 and $0.15 \mathrm{D}_{50}$. The apron extent in all directions was two times the flow depth.

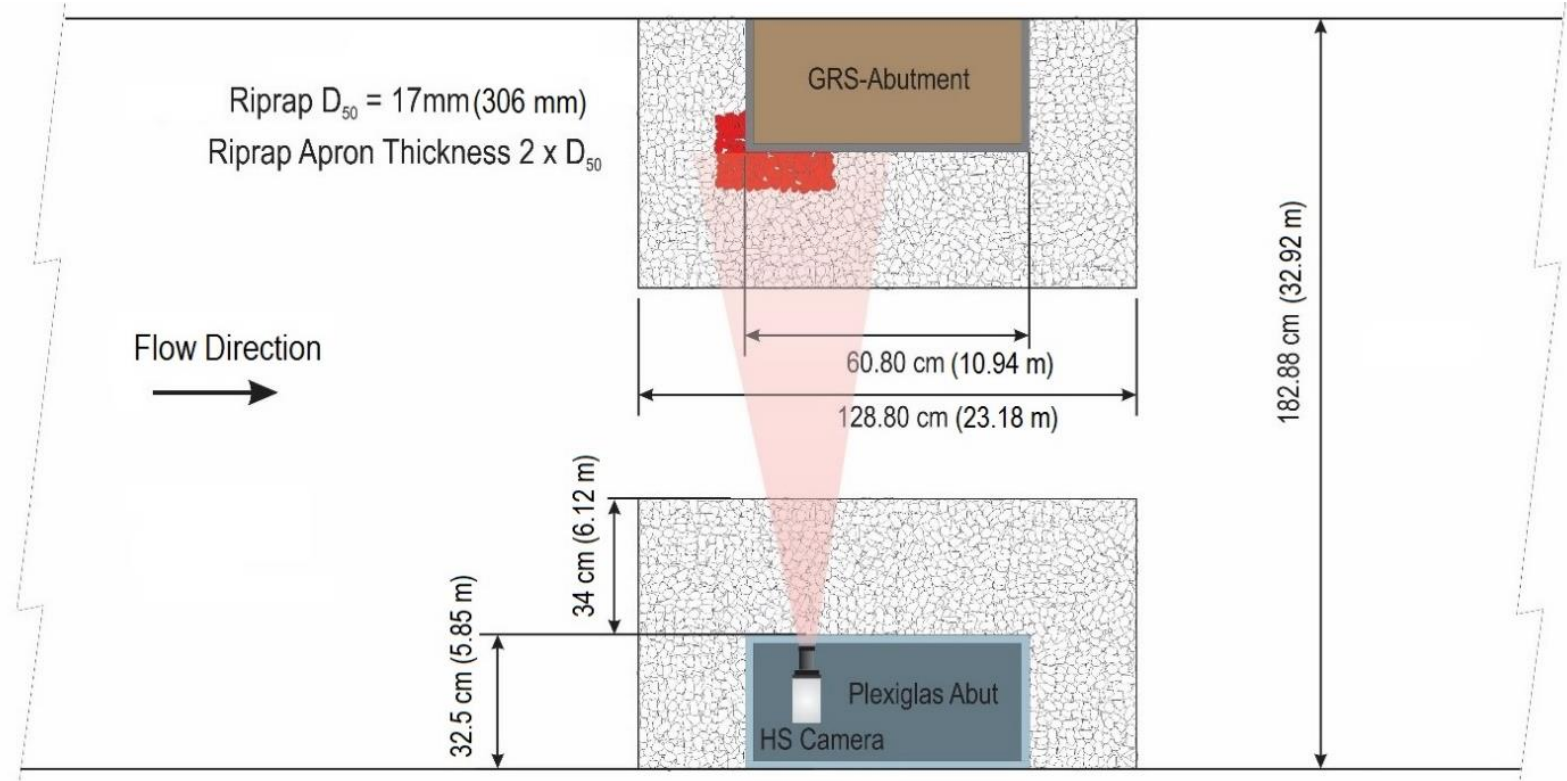

Figure 4.1: Plan View of Test Section

The rocks around the upstream corner of the monitored GRS abutment were colored with blue for the bottom first layer and red for the top second layer as shown in Figure 4.2. The colored rocks were placed in a potential "failure location" based on previous experiments from [11] [12]. A high speed camera was installed in the Plexiglas abutment to capture the incipient point of motion of the riprap installed around the GRS abutment. 

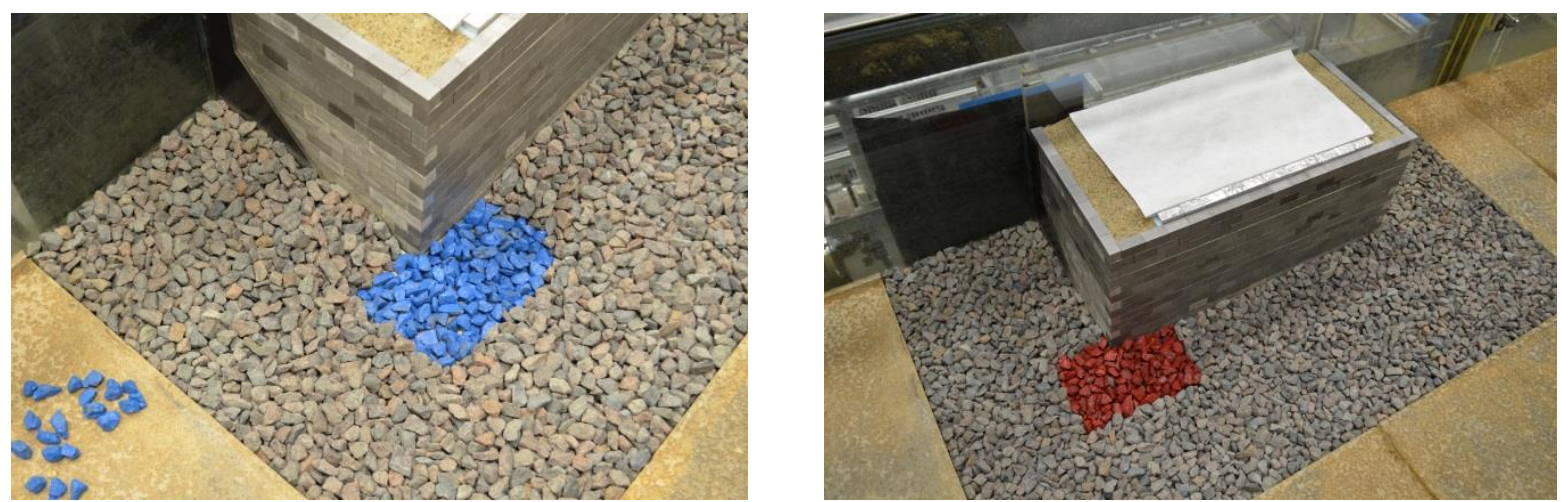

Figure 4.2: Installation of riprap layers (left) first layer (right) second layer

\subsubsection{Flow Conditions}

Relevant flow conditions are given in Table 4.1. The total running time for the experiment was 4.5 hours. The velocity was incremented until the rocks began to be entrained into the flow and the upstream flow depth was kept constant at $0.17 \mathrm{~m}$, although small fluctuations in the flow depth were impossible to avoid when the velocity was increased. The test was stopped when the riprap was stable in the failure zone, i.e. at the upstream corner of the abutment. Figure 4.3 shows the scanned bathymetry of rock layout after the test was finished. Deposition of the entrained riprap was observed in the downstream right behind the failure zone close to the abutment face. The experiment was repeated 2 times, for the first run the average upstream velocity for the first case was $0.42 \mathrm{~m} / \mathrm{s}$ when shear failure occurred and $0.44 \mathrm{~m} / \mathrm{s}$ for the second run. Table 4.1 presents the results of the tests.

Table 4.1: Flow conditions for experiments

\begin{tabular}{|c|c|c|c|}
\hline $\begin{array}{c}\text { Measured } \\
\text { Upstream Velocity } \\
\text { for a const. flow } \\
\text { depth } y_{1}=0.17 \mathrm{~m} \\
\text { V1 }[\mathrm{m} / \mathrm{s}]\end{array}$ & $\begin{array}{c}\text { Estimated }^{*} \\
\text { Average Velocity in } \\
\text { the Contraction } \\
V_{2}[\mathrm{~m} / \mathrm{s}]\end{array}$ & $\begin{array}{c}\text { Bridge Opening } \\
\text { Froude Number } \\
\text { Fr }\end{array}$ & Observations \\
\hline 0.27 & 0.52 & 0.4 & $\begin{array}{c}\text { Stable Riprap } \\
\text { (HEC 23 D.G. 14) } \\
\text { Design Velocity } V_{2} \text { for } \\
\text { Riprap } \mathrm{D}_{50}=17 \mathrm{~mm}\end{array}$ \\
\hline 0.39 & 0.76 & 0.59 & $\begin{array}{c}\text { Some rocks are shaking } \\
\text { but no entrainment } \\
\text { observed }\end{array}$ \\
\hline $0.42-0.44$ & 0.81 & $0.63-0.66$ & Shear Failure of Riprap \\
\hline
\end{tabular}

\subsubsection{Equilibrium Scour of Riprap Apron}

Figure 4.3 shows an isometric view and a top view of surface mapping results of the $D_{50}=17$ $\mathrm{mm}$ riprap apron after riprap shear failure and achievement of equilibrium scour depth. Most of the entrained rocks settled downstream along and near the abutment's front face. Failed riprap is shown in Figure 4.4. 


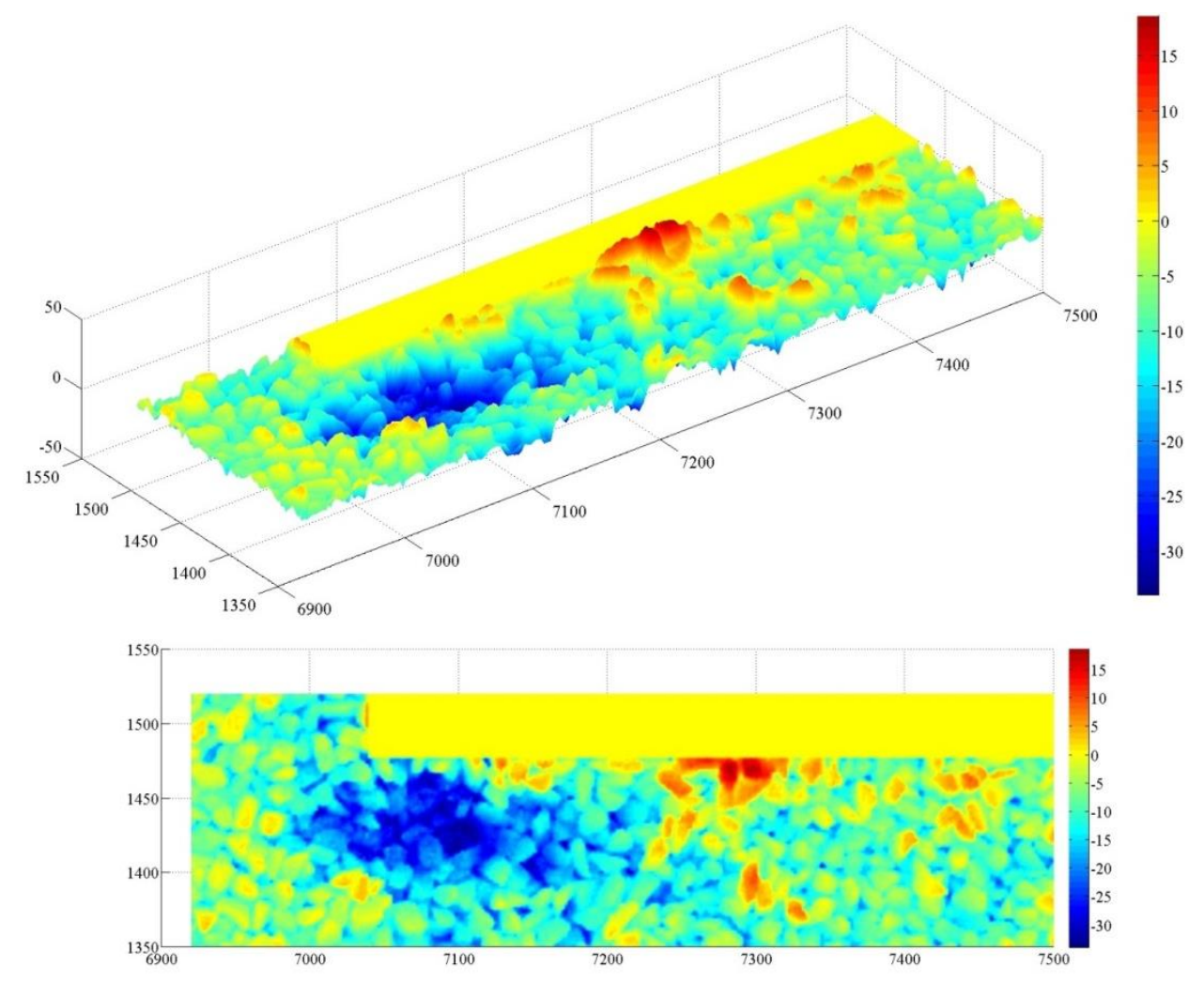

Figure 4.3: Scanned Bathymetry of rock layout after test was finished

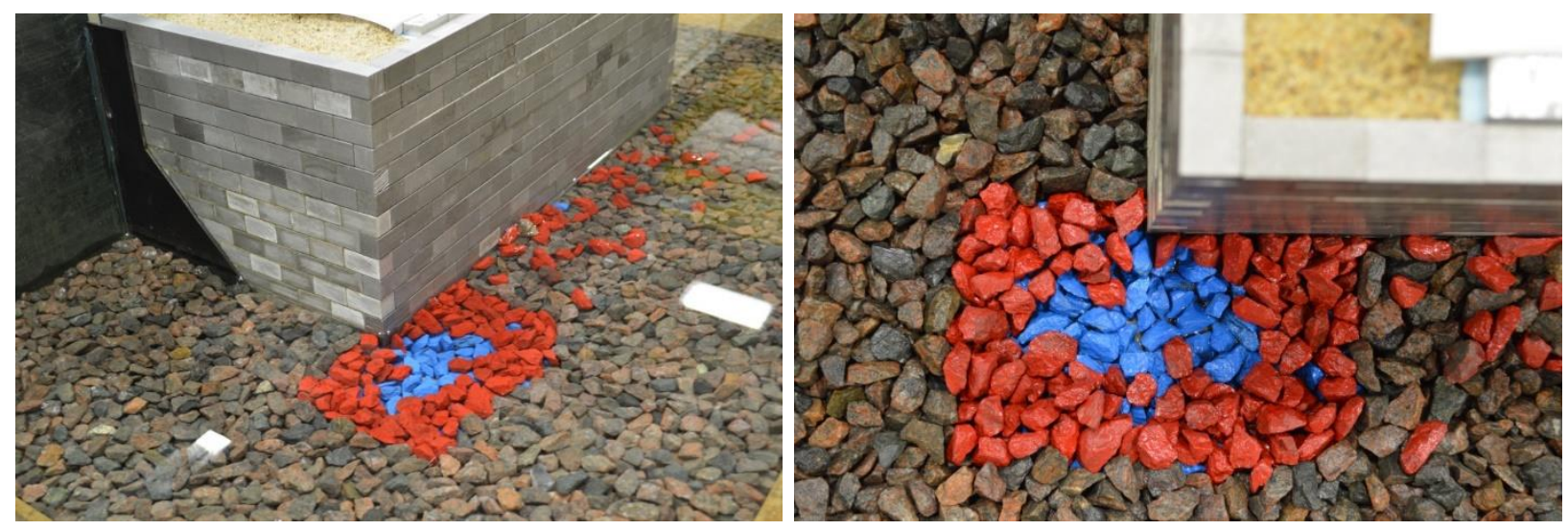

Figure 4.4: Failure of riprap at the upstream corner

\subsection{Numerical Modeling}

\subsubsection{Geometry of the Model}

The extent of the geometry in the numerical model comes from scaling the experimental setup dimensions by a factor of 18 . Figure 4.1 shows the scaled dimensions of the domain. In the numerical analysis only a half of this symmetrical domain was modeled to reduce the computational burden. 


\subsubsection{Flow Conditions}

When modelling rivers and other fluid with free surfaces the effect of gravity becomes important and the major governing non-dimensional number becomes the Froude (Fr) number. Thus, flow conditions in the simulation were calculated based on the Froude similarity with the experimental conditions. Table 4.2 lists the flow conditions in the simulation together with the predictions of instability and failure velocities for the full scale simulation.

Table 4.2: Flow conditions based on Froude similarity

\begin{tabular}{|c|c|c|c|}
\hline $\begin{array}{c}\text { Upstream Velocity } \\
\text { for a const. flow } \\
\text { depth y1=3.060 } \\
\text { V1 }[\mathbf{m} / \mathbf{s}]\end{array}$ & $\begin{array}{c}\text { Estimated* } \\
\text { Average Velocity in } \\
\text { the Contraction } \\
\text { V2 [m/s] }\end{array}$ & $\begin{array}{c}\text { Bridge Opening } \\
\text { Froude Number } \\
\text { Fr }\end{array}$ & For FSI Analysis \\
\hline 1.14 & 2.20 & 0.4 & $\begin{array}{c}\text { (HEC 23 D.G. 14) } \\
\text { Design Velocity V2 for } \\
\text { Riprap D } \text { Do }_{50} \text { 306mm }\end{array}$ \\
\hline 1.65 & 3.21 & 0.59 & $\begin{array}{c}\text { Potential Instability of } \\
\text { Riprap }\end{array}$ \\
\hline $1.78-1.87$ & $3.46-3.62$ & $0.63-0.66$ & Potential Shear Failure \\
\hline
\end{tabular}

\subsubsection{Model Development}

Before the construction of the geometry of the model, several simplifying assumptions had to be made. The exact representation of each rock in the computational model is not possible without an excessive amount of work. Also the randomness of the size distribution and the layout of the rocks are impossible to replicate with a reasonable amount of effort. The current implementation of the coupling mechanism requires the user to define many settings for each movable rock in STAR-CCM+ as well as the LS-DYNA model. Currently, this process cannot be automated and thus is very time consuming. Also, the structural mechanics part of the coupling relies on simulation restart capabilities in LS-DYNA that consume a significant amount of computer memory and time. The more moveable rocks there are in the model the longer it takes to restart the LS-DYNA job in each coupling time step. This, in fact, is one of the bottlenecks of the coupling mechanism and together with the domain remeshing in the CFD computation are the most time consuming elements of the coupled computation. For that reason, it was assumed that only 30 moveable rocks with their relative motion tracked would be a large enough sample. The fine mesh following all feature curves on the rocks is only retained for the moveable rocks and the mesh in CFD model is very dense around them and coarsens further away from them. Around 3,000 non-moveable rocks were placed in the testing area. Their packing is not as dense and random as it was in reality. In the model there is no easy option to drop them and allow for gravity forces to act to create a settled configuration. Although some graphical software allows for generation of 3 dimensional rock like shapes, the process is only based on simplified physics and there is still a need to perform LS-DYNA simulation for the stabilization of movable rocks in their initial equilibrium position. Thus, the non-moveable rocks were placed manually, leaving more void space between them than in reality due to the labor required to move them in $3 \mathrm{D}$ space and check their locations in relation to neighboring rocks. The domain was wrapped with the surface 
wrapper before the volume mesh was built. This closed many small gaps and eliminated problems with intersecting boundaries. The largest cells around the rocks far away from the moveable ones had their edge size around $75 \mathrm{~mm}$ while the smallest around the movable rocks were less than 5 $\mathrm{mm}$. These assumptions resulted in 4.4 million polyhedral cells in the CFD model. Figure 4.5 presents the mesh on the surface of the model.

The moveable rocks were placed sparsely covering the equivalent zone occupied by colored rocks in the experiment. All 30 rocks had the same shape but their orientation was randomized. The placement of the movable rocks could not be performed manually. They had to be dropped in LS-DYNA and their equilibrium positions had to be found via a finite element (FE) simulation. That way before the FSI simulation is started, they had proper contact with their neighbors and they didn't move any more if forces other than gravity weren't present in the system. The LSDYNA model was comprised of 1.3 million shell elements and 30 inertia elements attached to the center of gravity (CG) of the moveable rocks. The bed used in LS-DYNA was constructed from the wrapped surface obtained by exporting the STAR-CCM+ boundary mesh. Explicit time integration was used with a time step of 4.50E-06 sec. This drop simulation was run for 10 seconds of real time. That procedure took approximately 7 hours on 64 cores of the TRACC Zephyr cluster. The initial positions of moveable rocks as well as their final, equilibrium positions are shown in Figure 4.6. The movable rocks stay flush with the top surface of other rocks.

The surfaces describing the final positon of the moveable rocks were extracted from LSDYNA and imported to the STAR-CCM+ model. The shell elements used for moveable rocks in LS-DYNA, although represented by a zero thickness surface, have a thickness of $1 \mathrm{~mm}$. The contact definition in LS-DYNA doesn't allow for penetrations of these rocks into the bed. That assumption results in at least a $1 \mathrm{~mm}$ gap between the rocks and the bed in STAR-CCM+. This is needed to prevent any instabilities resulting from squeezing the computational cells in CFD to zero thickness in the case of contacts or collisions between movable rocks and the bed. After this step was completed a pure CFD analysis was performed for several inlet velocities: $1.7 \mathrm{~m} / \mathrm{s}, 1.6 \mathrm{~m} / \mathrm{s}, 1.5$ $\mathrm{m} / \mathrm{s}, 1.4 \mathrm{~m} / \mathrm{s}, 1.3 \mathrm{~m} / \mathrm{s}, 1.14 \mathrm{~m} / \mathrm{s}$ to create initial states for the FSI analysis. The unsteady RANS solver with a time step of 0.025 seconds was used for the CFD and the FSI analysis. The standard k-epsilon turbulence model was used in the analysis. The inlet velocity was assumed constant across the inlet boundary. An important simplifying assumption was disregarding the two phase nature of the experiment. In the simulations only one phase, water, was simulated. So called closed-lid simulations were performed instead of more realistic but significantly more computationally expensive as well as less robust Volume of Fluid (VOF) simulations with a free surface. This assumption causes the water height in the model to stay constant and uses a symmetry boundary condition at the water surface. In the contraction zone the water level would rise slightly and an effective cross section would be slightly larger in the experiment. These differences were assumed to be minor. The closed lid approach was compared with the VOF approach for this particular case and turned out to be conservative (See section 4.2.4 for explanation). 

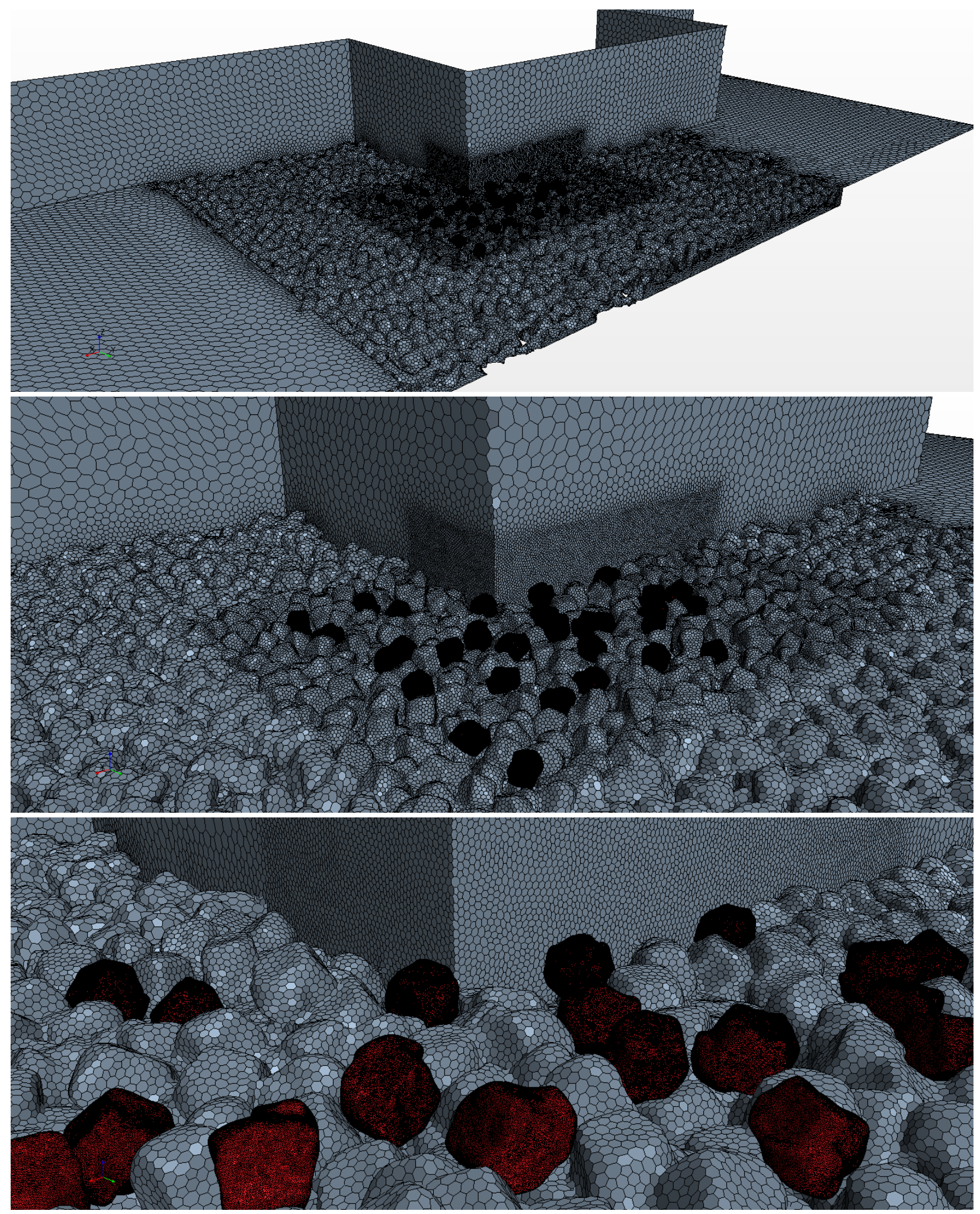

Figure 4.5: The layout of the rocks in the CFD model 

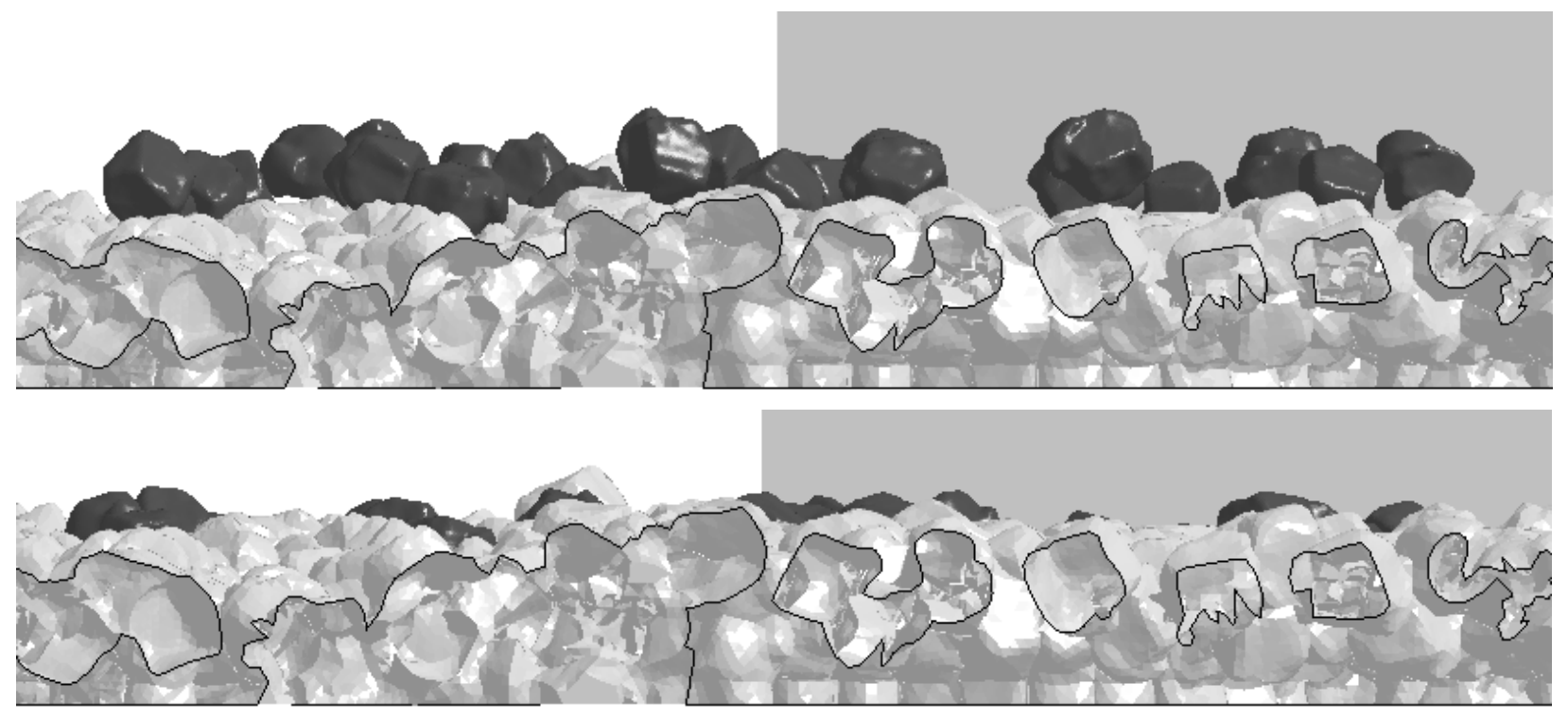

Figure 4.6: Pre drop and final position of the movable rocks

\subsubsection{Results of CFD Analysis}

In the CFD analysis forces on the individual moveable rocks were plotted. Once they stabilized (which occurred in about 5 seconds of simulated time) the simulation was stopped and the time was reset to zero while the field variables were retained for further FSI analysis. That way both the CFD and CSM analyses were properly initialized before the FSI computation was started.

The results of CFD analysis with inlet velocity of $1.3 \mathrm{~m} / \mathrm{s}$ is presented below in more detail. This is a threshold velocity for which failure of riprap is detected. For the other cases, similar conclusions can be drawn. Figure 4.7 shows a velocity distribution just above the riprap rocks in the simulation with closed lid approach. Although the average velocity in the contraction zone for this case is around $2.5 \mathrm{~m} / \mathrm{s}$, locally the water velocity can be as high as $4.3 \mathrm{~m} / \mathrm{s}$ near the corner of the abutment. Figure 4.8 shows a similar cross section for a model where the interface between water and the air was resolved for in a VOF simulation. Maximum velocities near the movable rocks in that simulation are slightly smaller as compared to the velocities from closed lid simulation. At first this is counterintuitive as the water level in reality (and VOF simulation) drops in the contraction causing the water to accelerate. However, in the immediate upstream of the abutment corner the water level is higher than in the closed lid approach, and that causes the water velocity to be initially lower than in the closed lid simulation. The closed lid approach is forcing the same amount of water to flow through a smaller channel than in the case of free surface flow, causing rocks to be set in motion at a slightly lower approach water velocity near the abutment corner. Further downstream the velocity is higher in the VOF simulation, but this is beyond the area of interest and the location of movable rocks. Figure 4.9 shows a cut through the domain along the flow with highlighted water surface level in both types of simulations. 
Figure 4.10 shows a perspective view of the abutment at the end of the CFD simulation. The pressure is plotted on the surface of the moveable rocks. Ten rocks with the highest forces acting on them are labeled in the figure. All of them reside by the inlet to the contraction zone where water acceleration is present. Figure 4.11 shows the histories of X (along the flow) and (Z) vertical forces on the moveable rocks. Rocks 5 and 16 have the highest forces acting on them. Figure 4.11 shows the final values of the $\mathrm{X}$ and $\mathrm{Z}$ forces on ten most critical rocks labeled earlier in Figure 4.10. A resultant force is also computed from these two components. The third $\mathrm{Y}$ component is disregarded here as it is significantly smaller. The resultant forces on rocks 5, 16, and 21 are 291, 299, and $261 \mathrm{~N}$ respectively. The weight of the rocks is about $354 \mathrm{~N}$. With the resultant force acting on the most critical rocks approaching $300 \mathrm{~N}$, some motion of the rocks is expected in the model. Their positioning with respect to the other rocks, boundaries, and mutual interlocking is a major deciding factor that determines if the rocks will roll out from their initial positions or not. This question can only be answered through the FSI analysis where collisions and contacts between all the rocks are taken into account.

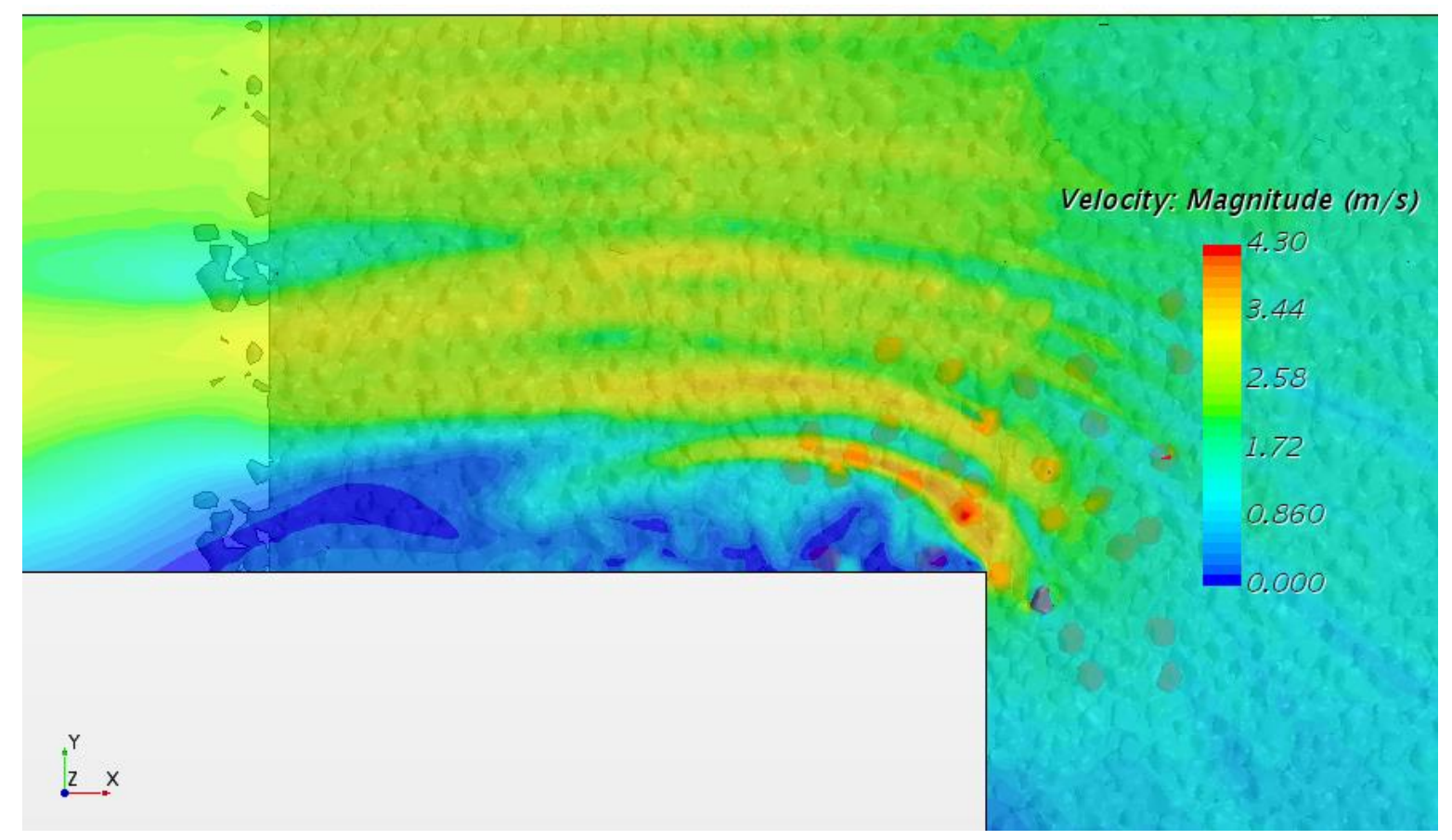

Figure 4.7: Velocity profile in a cross section just above the riprap rocks for the case with inlet velocity of $1.3 \mathrm{~m} / \mathrm{s}$ in closed lid simulation 


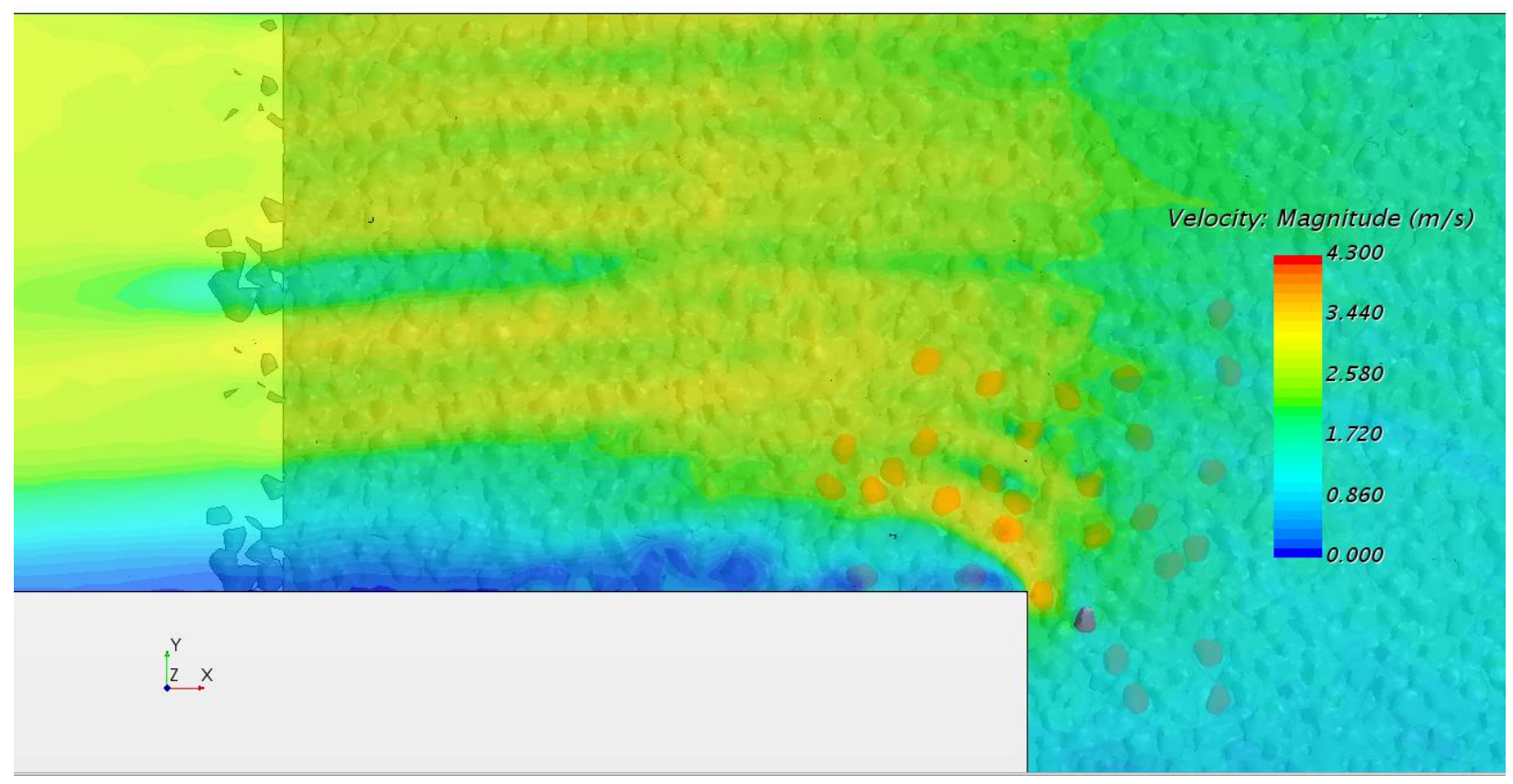

Figure 4.8: Velocity profile in a cross section just above the riprap rocks for the case with inlet velocity of $1.3 \mathrm{~m} / \mathrm{s}$ in VOF model

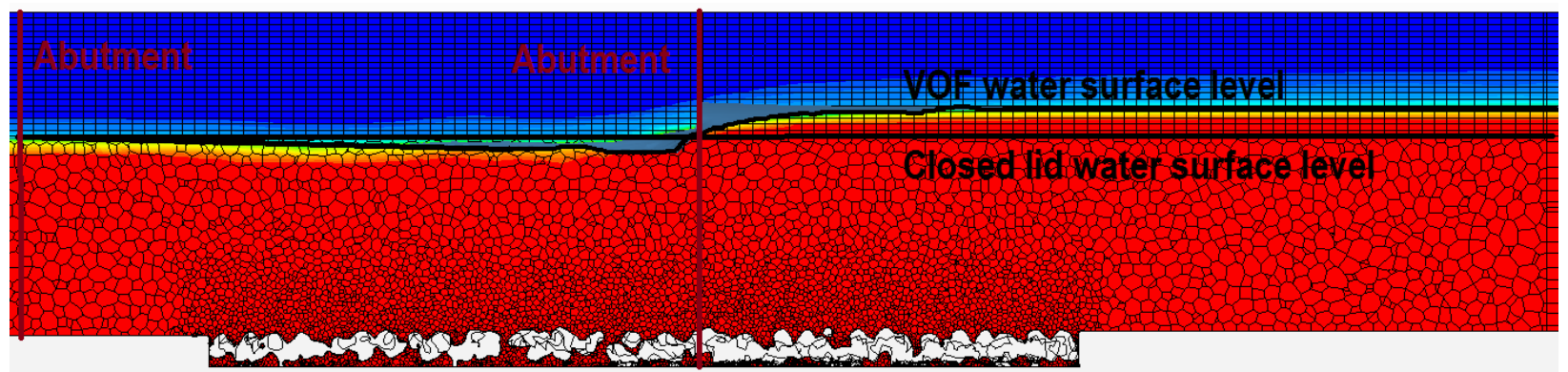

Figure 4.9: Water surface location for the VOF and closed lid simulation 


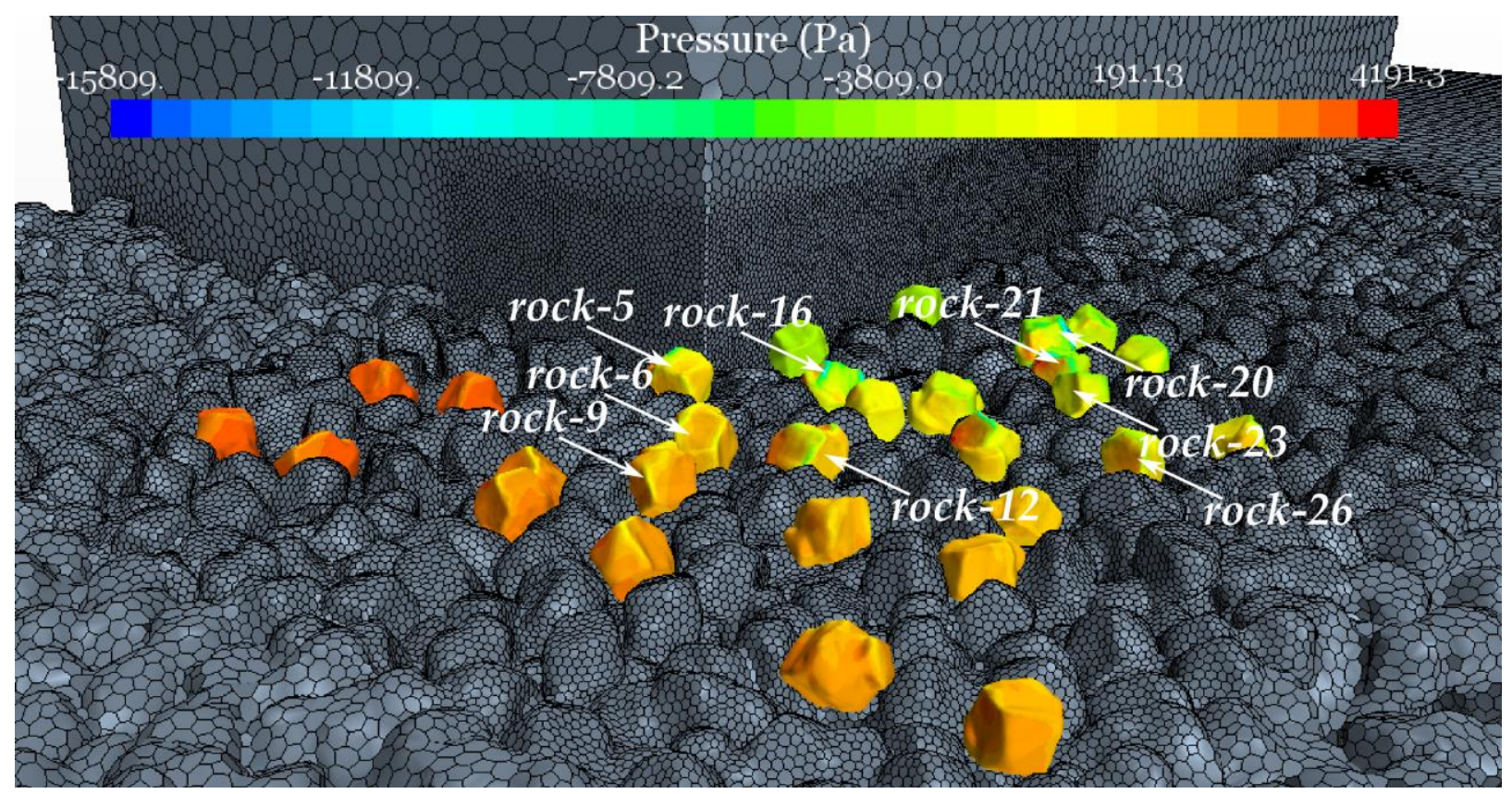

Figure 4.10: Location of the rocks with the highest forces in CFD analysis

It should be noted that the $\mathrm{Z}$ component of the force is quite substantial. It comes from both the buoyancy effect as well as from the fact that there is a significant flow in the voids between the non-moveable rocks. As pointed earlier, the packing density of the rocks is smaller than the density in the real experiment. Also their shape is smoothed more due to larger cell sizes used away from the moveable rocks. This is also a conservative simplification of the model. It is important to point out that to compute the forces acting on the rocks, both the pressure and the shear components were taken into account. However, the pressure components are significantly higher for all the rocks. The shear forces are between two to three orders of magnitude smaller than the forces resulting from the pressure drag.

The FSI simulations were performed with a coupling time step of $0.025 \mathrm{sec}$, which was also the time step in the CFD portion of the procedure. The time step in LS-DYNA was significantly smaller in order to account for the collisions and contacts between the moveable and stationary rocks. Just like in the drop procedure for initial movable rock positioning, it was 4.50E-o6 sec. That means for each time step in STAR-CCM+ there were around 5,550 steps in LS-DYNA. Ideally these steps should be either equal or inner iterations within one time step should be performed in order to get better accuracy. However, if 5 inner iterations within a time step are needed, then the overall computation time increases accordingly 5 times. For the validation studies, each simulation took about one week of computation time. This is a reasonable time for production runs. However, 5 weeks per run would be unreasonable and debugging such simulations would take months. The assumed approach is believed to be a good compromise between accuracy, efficiency, and obtaining conservative results that can be used in engineering design. 

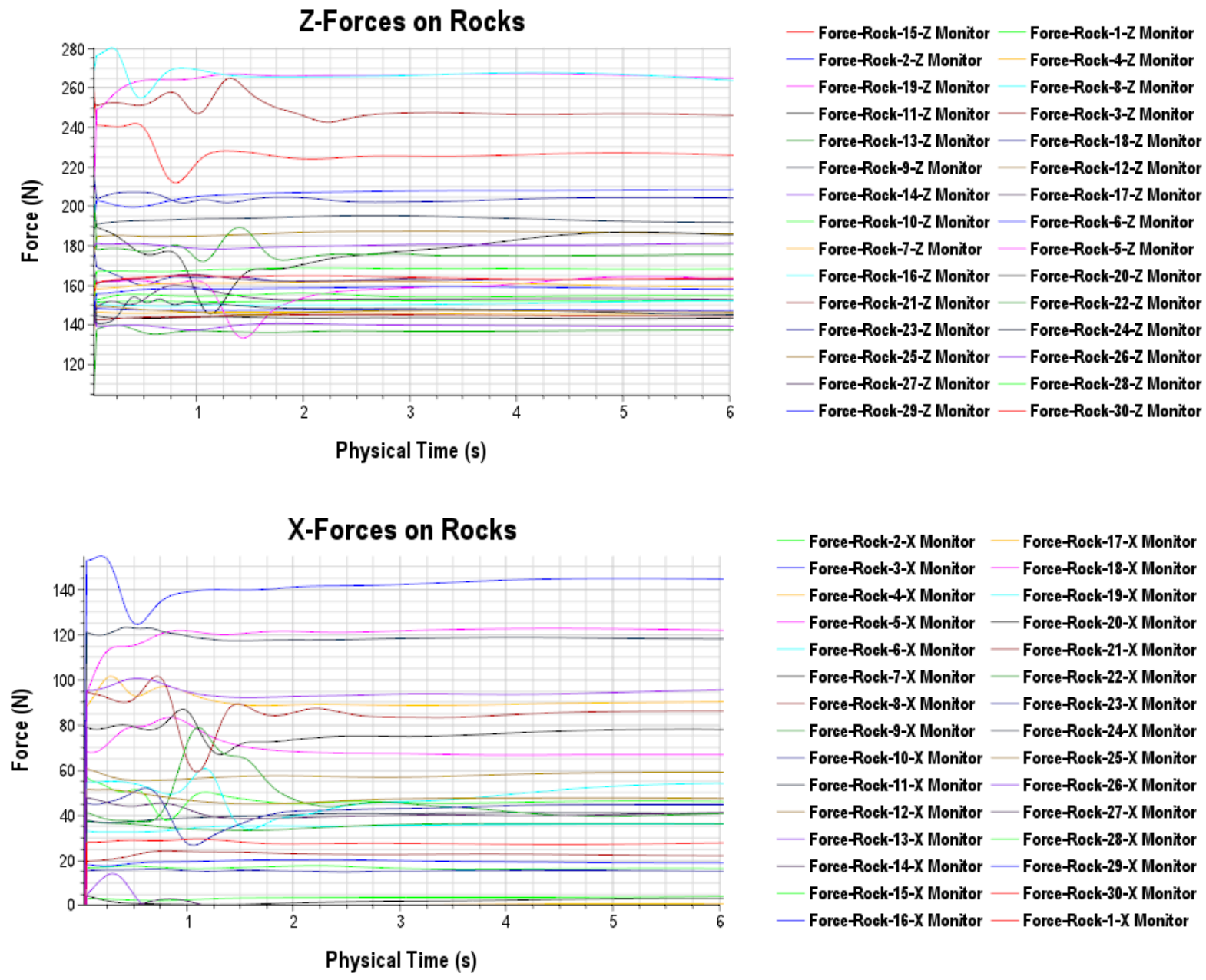

Figure 4.11: Forces on the moveable rocks in the simulation with inlet velocity of $1.3 \mathrm{~m} / \mathrm{s}$

\subsubsection{Results of FSI Analysis}

Figure 4.12 shows several snapshots from the FSI simulation for the case with inlet velocity of $1.3 \mathrm{~m} / \mathrm{s}$. Three rocks with the highest forces on them are labeled. Rock 5 is positioned near the abutment corner and it is not moving in the FSI simulation at all. The other rocks experience a sudden push when they are "allowed" to move at the beginning of the FSI simulation. That causes them to wiggle a little in their positions but eventually they come back to the initial positions adjusted for the hydrodynamic forces introduced in the FSI simulation. 
Table 4.3: Forces on the critical rocks in the simulation with inlet velocity of $1.3 \mathrm{~m} / \mathrm{s}$

\begin{tabular}{|c|c|c|c|}
\hline Rock \# & Z Force & X Force & Resultant \\
\hline $\mathbf{1 6}$ & 262 & 145 & 299 \\
\hline $\mathbf{5}$ & 264 & 122 & 291 \\
\hline $\mathbf{2 1}$ & 246 & 86 & 261 \\
\hline $\mathbf{1 5}$ & 225 & 46 & 230 \\
\hline $\mathbf{6}$ & 208 & 36 & 211 \\
\hline $\mathbf{2 3}$ & 204 & 45 & 208 \\
\hline $\mathbf{2 6}$ & 181 & 95 & 204 \\
\hline $\mathbf{2 0}$ & 185 & 78 & 200 \\
\hline $\mathbf{1 2}$ & 186 & 59 & 195 \\
\hline $\mathbf{9}$ & 191 & 37 & 194 \\
\hline
\end{tabular}

The trajectories of centers of gravity of the rocks that moved the most is marked in red Figure 4.13. 


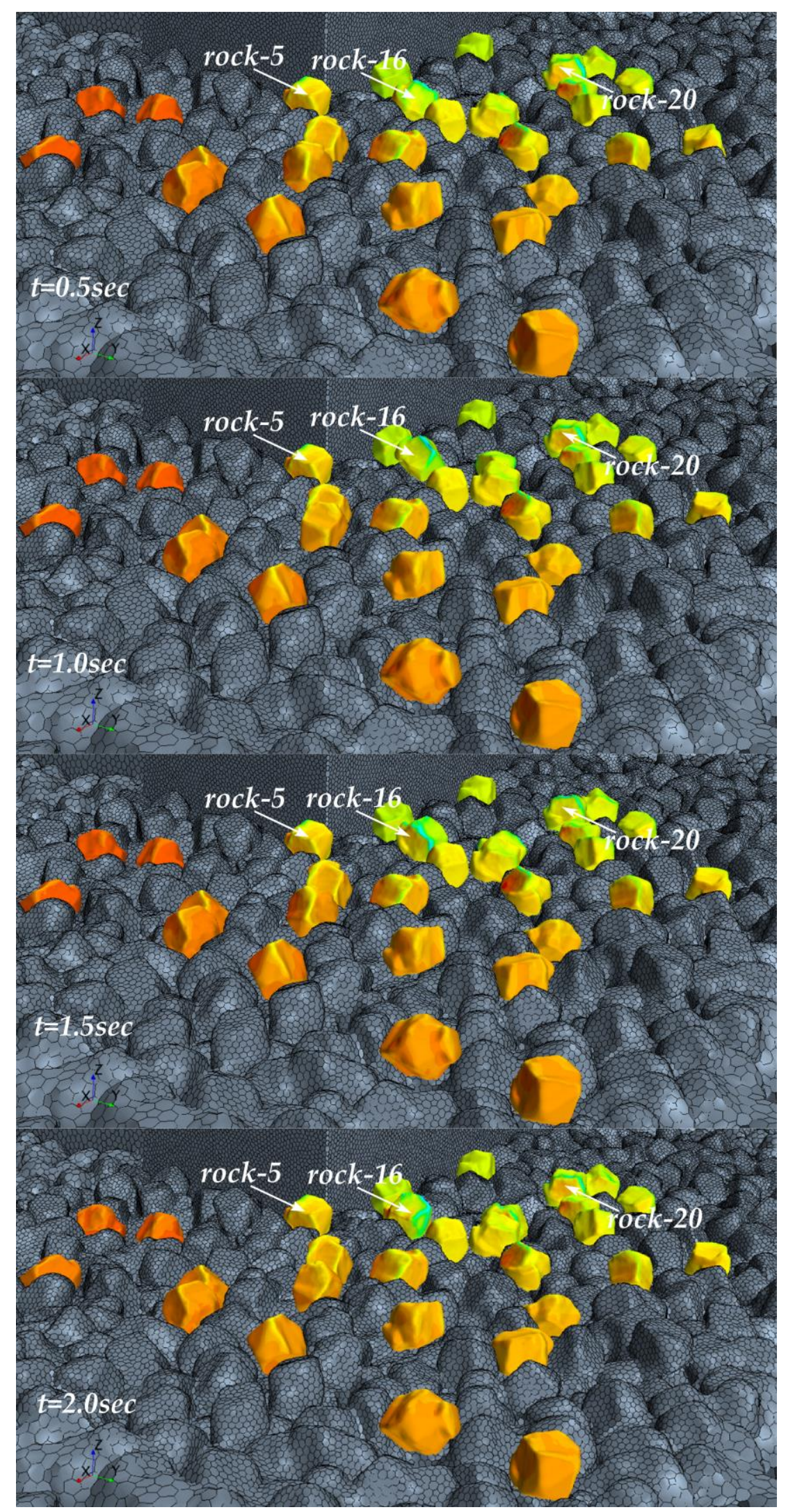

Figure 4.12: Snapshots of the FSI simulation for inlet velocity of $1.3 \mathrm{~m} / \mathrm{s}$ 


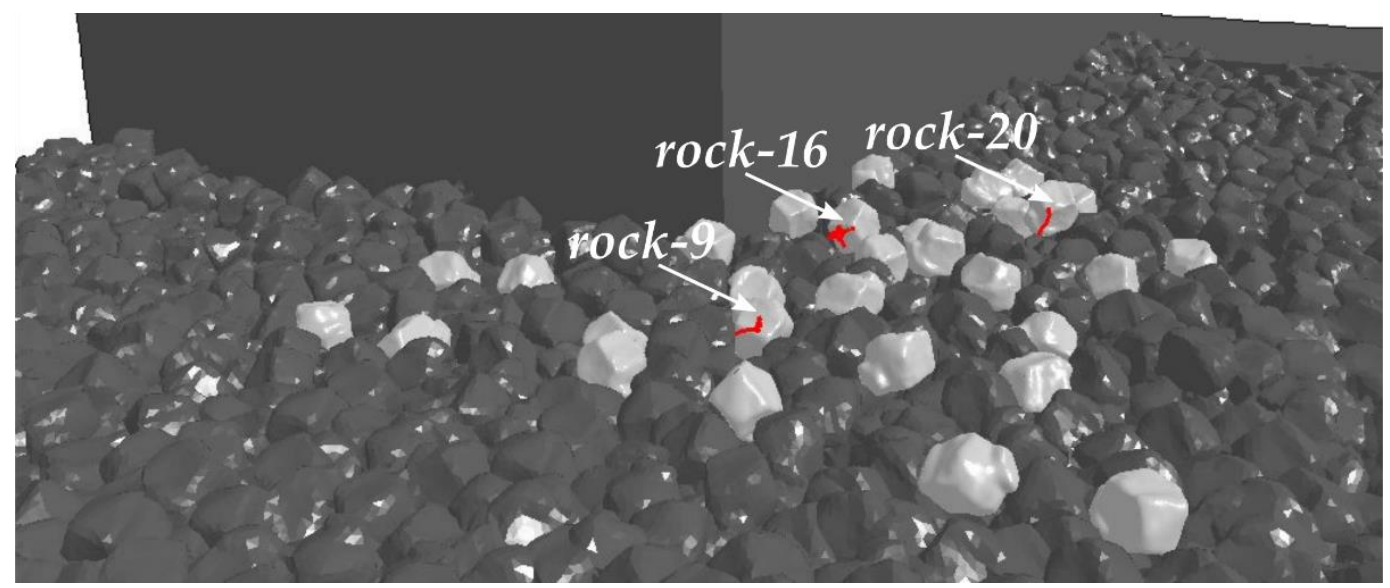

Figure 4.13: Position of rocks at $2.0 \mathrm{sec}$ in FSI simulation for inlet velocity of $1.3 \mathrm{~m} / \mathrm{s}$

The next analyzed case was the case with the inlet velocity of $1.4 \mathrm{~m} / \mathrm{s}$. Forces on the most critical rocks reported at the end of the stationary CFD simulation are shown in Table 4.4. They are in the exact same order as in the case with the inlet velocity of $1.3 \mathrm{~m} / \mathrm{s}$. The resultant forces increased between 3 to $10 \%$. In the FSI analysis, significantly more rocks started to move and leave their initial positions. Figure 4.15 shows several snapshots from the FSI simulation for that case. The rocks labeled 9, 15, 16, and 23 were the ones with the most noticeable movement. Several other rocks around them were wiggling as well. Collisions of the rolling rocks with the surrounding ones triggered local motion of several more rocks. The trajectories of centers of gravity of the rocks that moved the most is marked in red Figure 4.14. This case can be considered as the borderline between unstable and failing riprap.

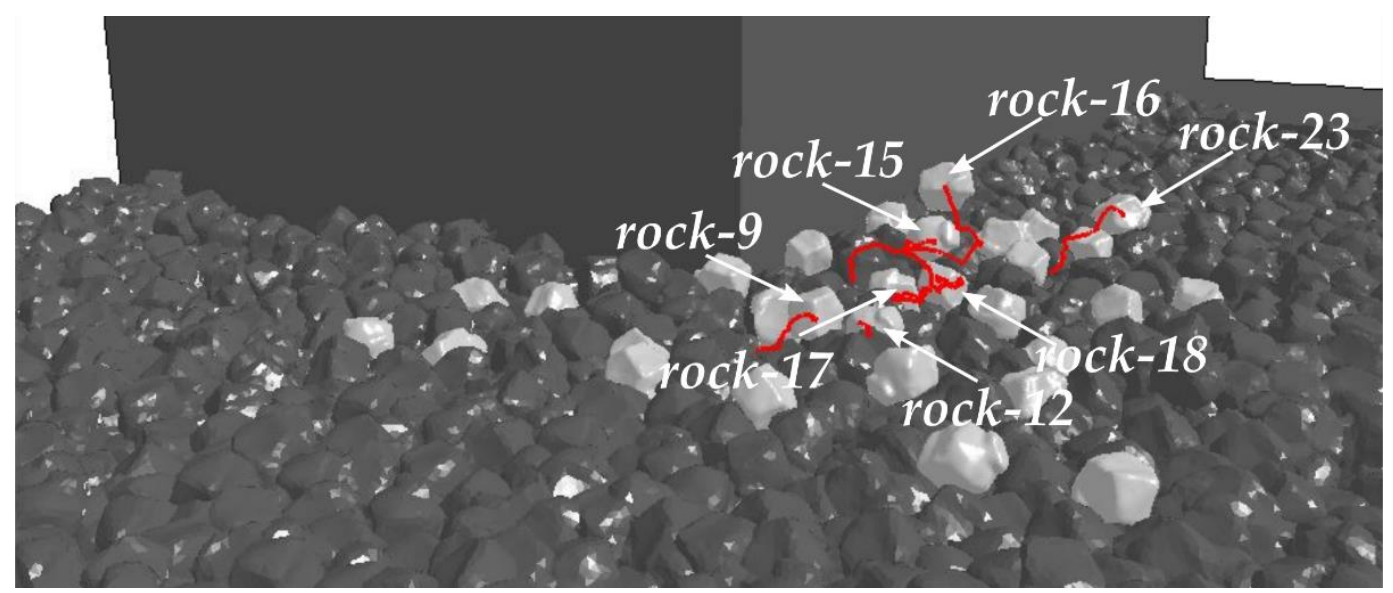

Figure 4.14: Position of rocks at $2.0 \mathrm{sec}$ in the FSI simulation for inlet velocity of $1.4 \mathrm{~m} / \mathrm{s}$ 
Table 4.4: Forces on the critical rocks in the simulation with inlet velocity of $1.4 \mathrm{~m} / \mathrm{s}$

\begin{tabular}{|c|c|c|c|}
\hline Rock \# & Z Force & X Force & Resultant \\
\hline $\mathbf{1 6}$ & 289 & 160 & 330 \\
\hline $\mathbf{5}$ & 285 & 143 & 319 \\
\hline $\mathbf{2 1}$ & 263 & 101 & 282 \\
\hline $\mathbf{1 5}$ & 249 & 61 & 257 \\
\hline $\mathbf{6}$ & 219 & 41 & 222 \\
\hline $\mathbf{2 3}$ & 215 & 52 & 221 \\
\hline $\mathbf{2 6}$ & 190 & 112 & 220 \\
\hline $\mathbf{2 0}$ & 191 & 88 & 210 \\
\hline $\mathbf{1 2}$ & 193 & 68 & 205 \\
\hline $\mathbf{9}$ & 198 & 42 & 202 \\
\hline
\end{tabular}

In the case with an inlet velocity of $1.5 \mathrm{~m} / \mathrm{s}$, the failure of the riprap became even more apparent. Table 4.5 shows forces acting on the most critical rocks in that simulation. The maximum forces grew by additional $10 \%$. Figure 4.16 shows snapshots from that simulation. The rocks with most noticeable motions, $9,15,16,18,23$, and 26 are labeled in this figure. Additionally rocks $6,12,18$, and 20 become quite unstable and move locally. The trajectories of centers of gravity of the rocks that moved the most in that case is marked in red Figure 4.17.

Table 4.5: Forces on the critical rocks in the simulation with inlet velocity of $1.5 \mathrm{~m} / \mathrm{s}$

\begin{tabular}{|c|c|c|c|}
\hline Rock \# & Z Force & X Force & Resultant \\
\hline $\mathbf{1 6}$ & 315 & 194 & 370 \\
\hline $\mathbf{5}$ & 309 & 168 & 352 \\
\hline $\mathbf{2 1}$ & 282 & 119 & 306 \\
\hline $\mathbf{1 5}$ & 271 & 68 & 279 \\
\hline $\mathbf{6}$ & 232 & 48 & 237 \\
\hline $\mathbf{2 3}$ & 230 & 58 & 237 \\
\hline $\mathbf{2 0}$ & 210 & 109 & 236 \\
\hline $\mathbf{2 6}$ & 196 & 128 & 234 \\
\hline $\mathbf{9}$ & 214 & 48 & 220 \\
\hline $\mathbf{1 2}$ & 203 & 78 & 217 \\
\hline
\end{tabular}




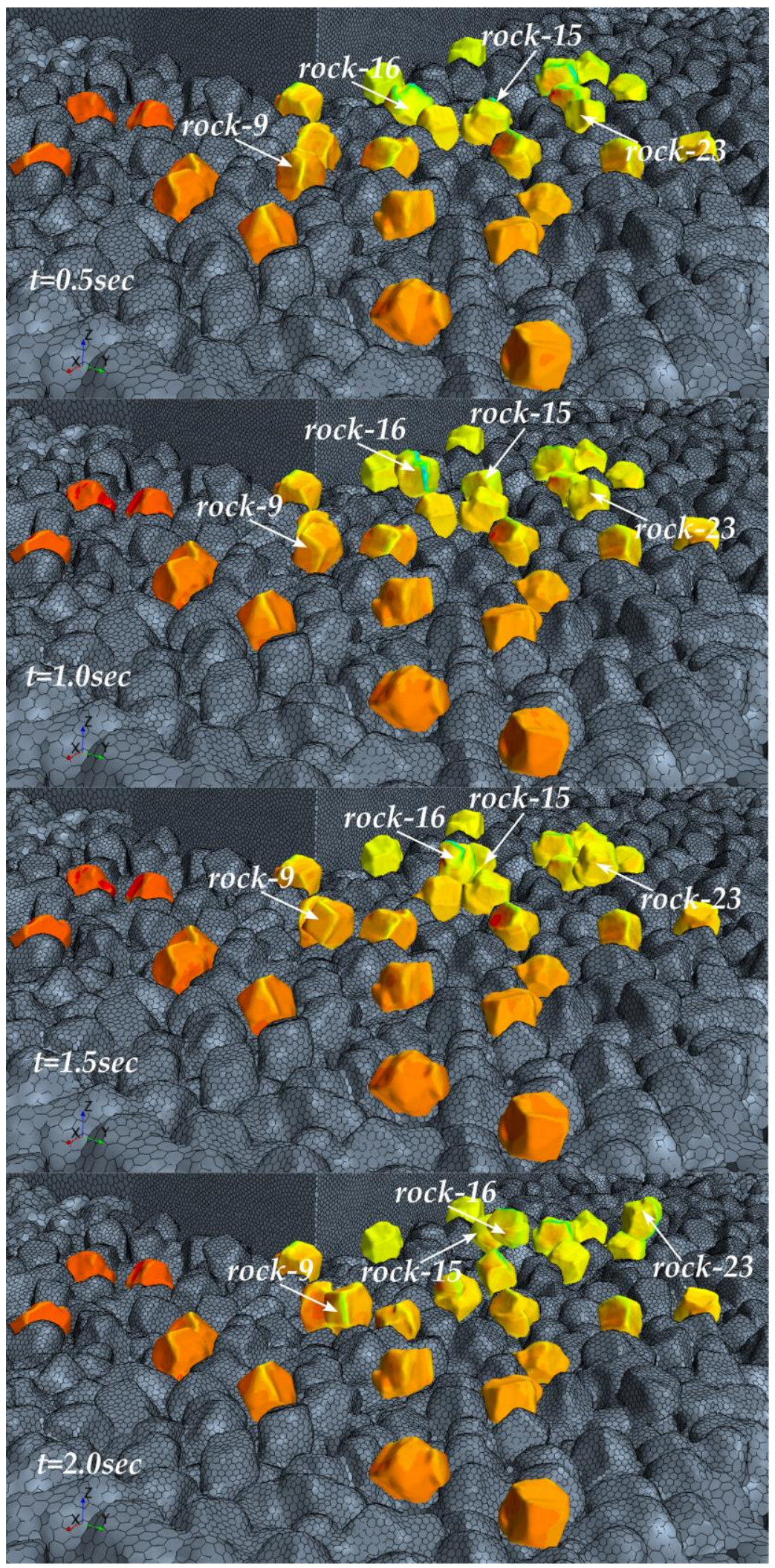

Figure 4.15: Snapshots of the FSI simulation for inlet velocity of $1.4 \mathrm{~m} / \mathrm{s}$ 


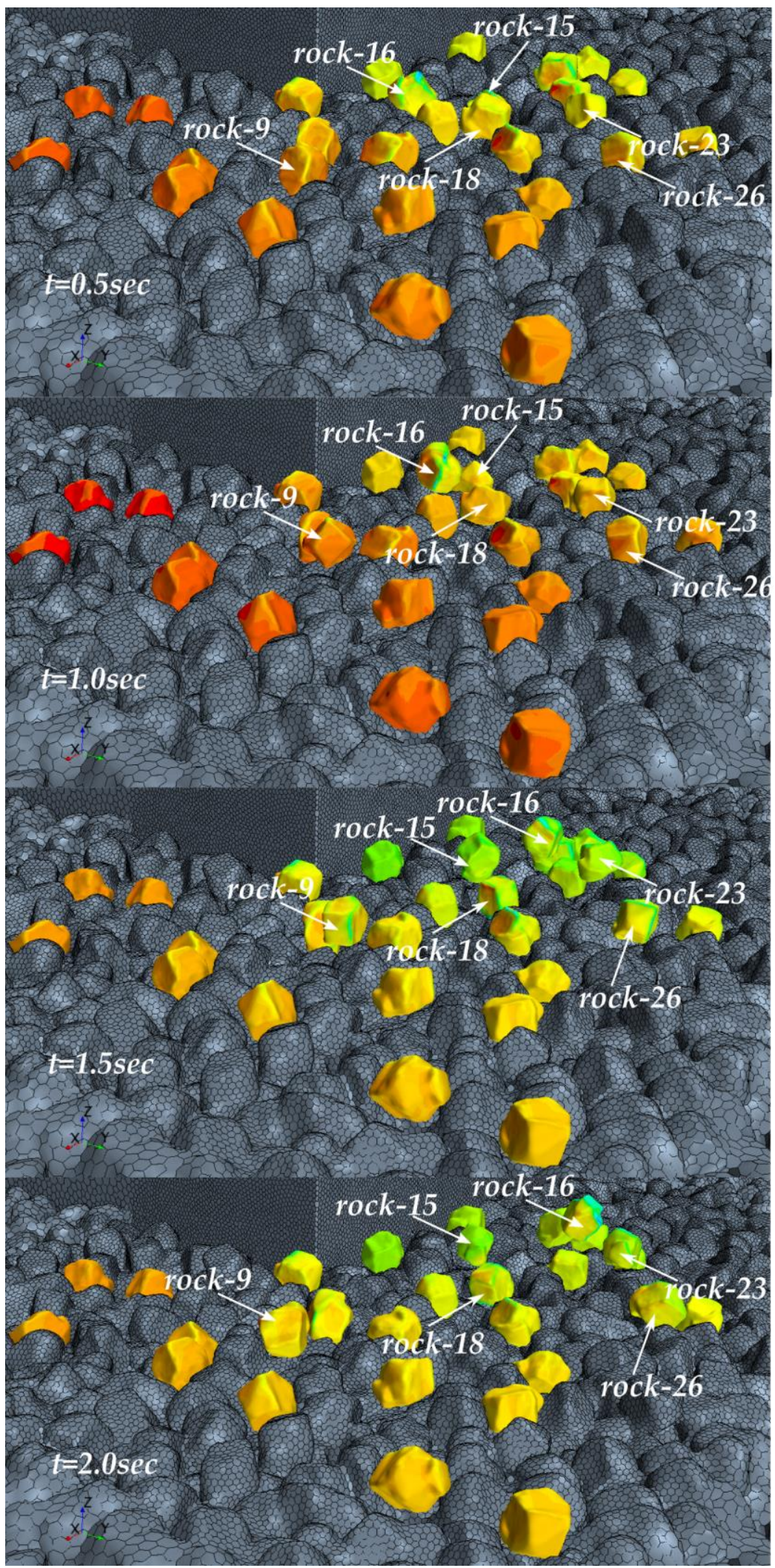

Figure 4.16: Snapshots of the FSI simulation for inlet velocity of $1.5 \mathrm{~m} / \mathrm{s}$ 


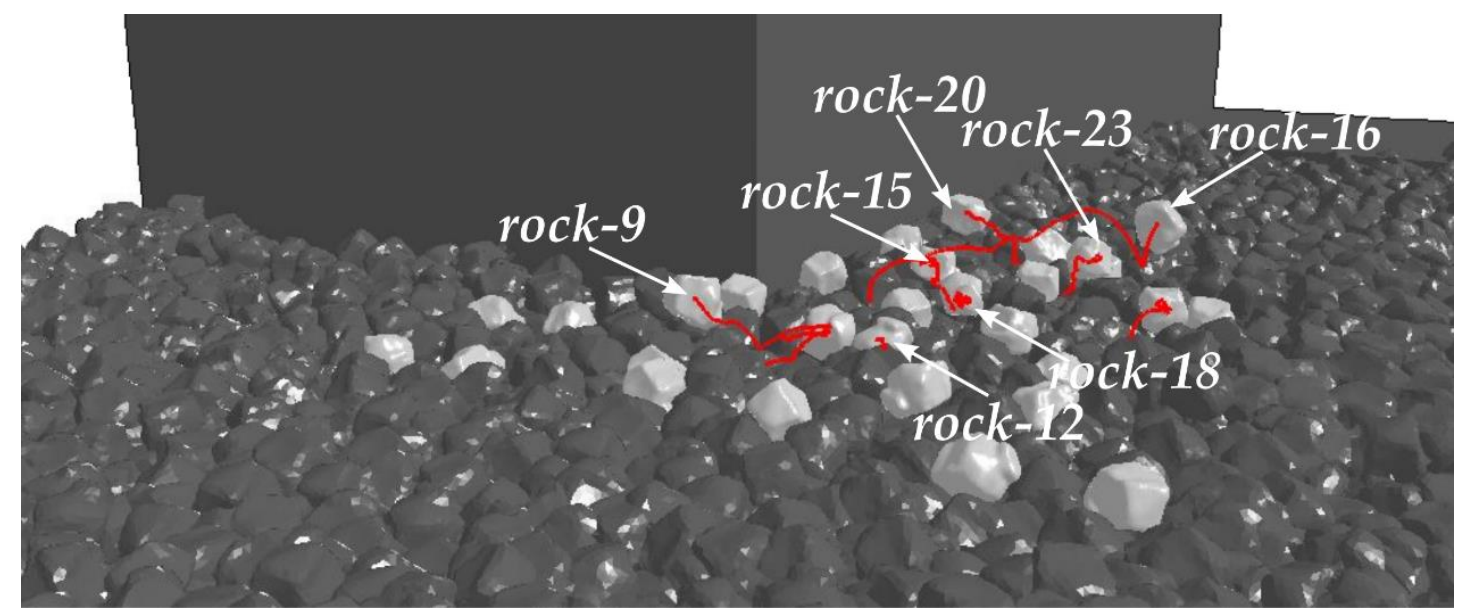

Figure 4.17: Position of rocks at $2.0 \mathrm{sec}$ in the FSI simulation for inlet velocity of $1.5 \mathrm{~m} / \mathrm{s}$

The scaled experimental results predict that the riprap should be stable at the inlet velocity of $1.14 \mathrm{~m} / \mathrm{s}$. In the simulation the rocks do not move from their initial positions. Minimal initial vibrations were present for a short period of time, which was caused by sudden application of new forces (drag) in the FSI simulation. The rocks stay stable until the inlet velocity is increased to 1.4 $\mathrm{m} / \mathrm{s}$. At this velocity, four rocks leave then their initial positions and several more are unstable and move locally. It can be concluded that the instability of riprap rocks occurs around $1.3 \mathrm{~m} / \mathrm{s}$ and the failure of the riprap occurs between 1.3 and $1.4 \mathrm{~m} / \mathrm{s}$. Table 4.6 compares scaled results from the experiments with the results from the simulations. The simulated velocity of $1.3 \mathrm{~m} / \mathrm{s}$ is $21 \%$ lower than the equivalent scaled velocity of $1.65 \mathrm{~m} / \mathrm{s}$ in the experiment. The simulated failure velocity $1.3 \mathrm{~m} / \mathrm{s}-1.4 \mathrm{~m} / \mathrm{s}$ is around $25 \%$ lower than the equivalent range of velocities from the experiment. This set of simulations indicates that the computational approach is valid and conservative. It is believed that the difference between the simulation and the experiment can be further decreased by more detailed mapping of the bed rocks.

Table 4.6: Comparison of predicted failure upstream velocities from experiments and simulations

\begin{tabular}{|c|c|c|c|}
\hline $\begin{array}{c}\text { Upstream Velocity } \\
\text { for a const. flow } \\
\text { depth y1=3.060 } \mathbf{~ m} \\
\text { V1 [m/s] }\end{array}$ & $\begin{array}{c}\text { Prediction from } \\
\text { experiment }\end{array}$ & $\begin{array}{c}\text { Upstream Velocity } \\
\text { for a const. flow } \\
\text { depth y1=3.060 } \mathbf{~ m} \\
\text { V1 [m/s] }\end{array}$ & $\begin{array}{c}\text { Prediction from } \\
\text { simulation }\end{array}$ \\
\hline 1.14 & $\begin{array}{c}\text { Design velocity, stable } \\
\text { riprap }\end{array}$ & 1.14 & Stable riprap \\
\hline 1.65 & $\begin{array}{c}\text { Potential Instability of } \\
\text { Riprap }\end{array}$ & 1.3 & $\begin{array}{c}\text { Potential Instability of } \\
\text { Riprap }\end{array}$ \\
\hline $1.78-1.87$ & Potential Riprap Failure & $1.3-1.4$ & Potential Riprap Failure \\
\hline
\end{tabular}




\section{Application of FSI Coupling to Analysis of the Middle Fork Feather River Bridge Riprap Installation}

\subsection{Site Conditions and Riprap Risk Assessment}

Before the installation of the riprap in 2011, a field survey was conducted on the bridge site. It included a boat survey of the riverbed bathymetry and acquiring of GPS coordinates of ground features near the bridge location. The boat survey was conducted using the SonarMite depth finder by Seafloor Systems. Figure 5.1 shows the location of the measurement points on the riverbed and the terrain around the bridge. Figure 5.2 shows elevation of the riverbed model built based on that data with the deepest point in front of the pier at elevation of about $4319 \mathrm{ft}$.

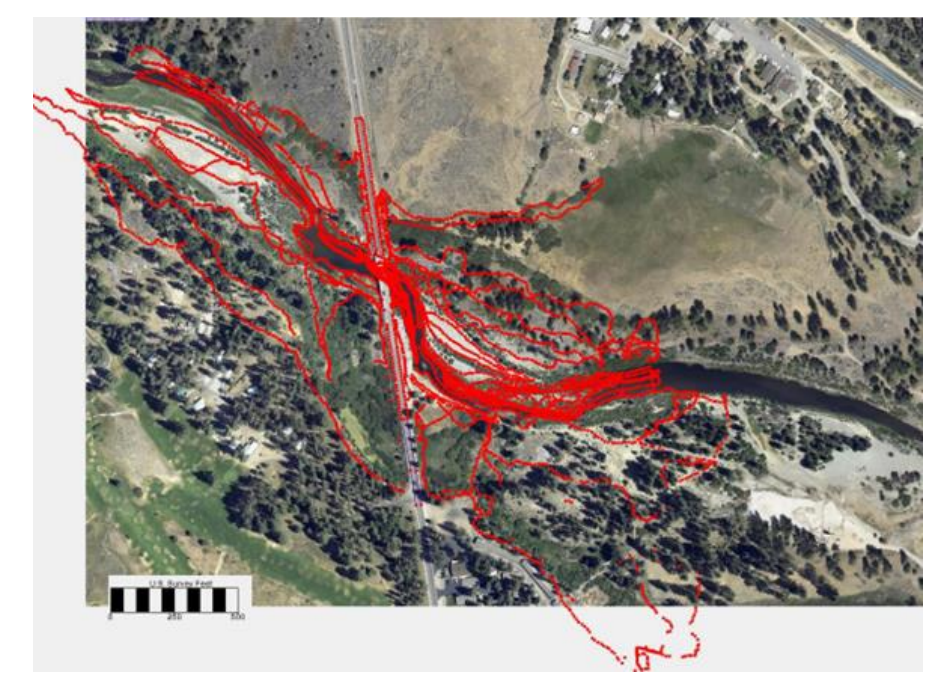

Figure 5.1: Boat survey and GPS points in the bridge vicinity [1]

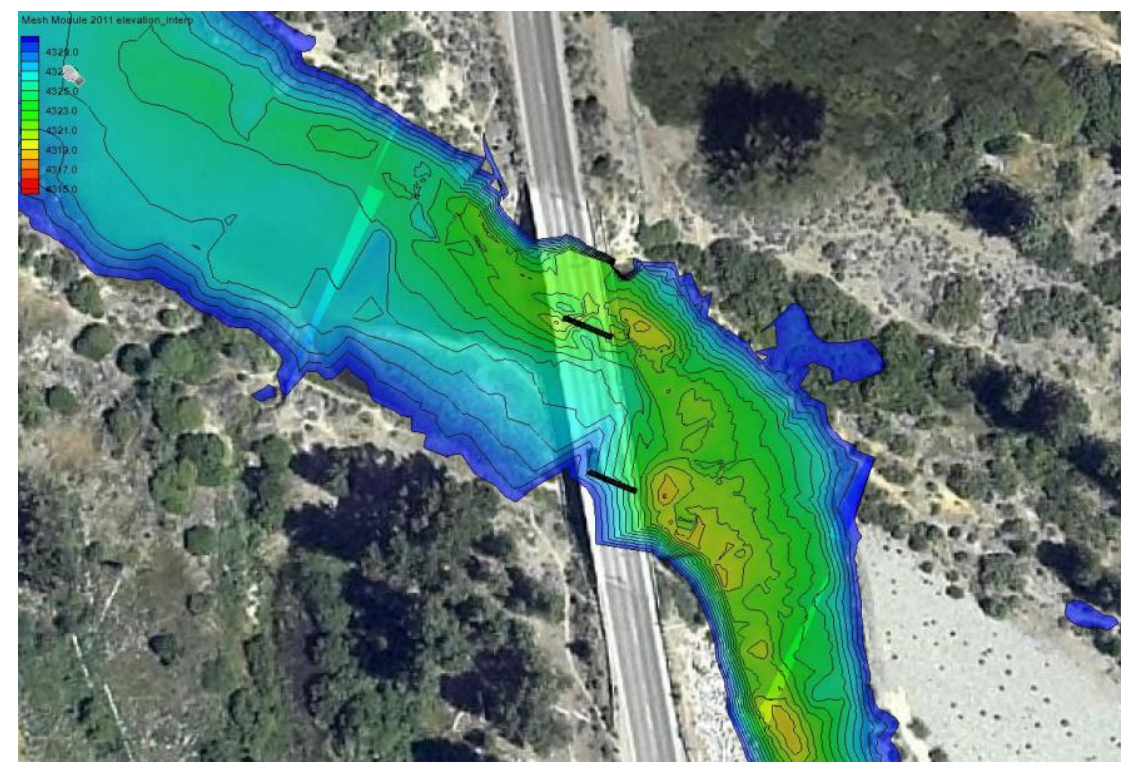

Figure 5.2: Bathymetry of the river before installation of the riprap (2011) [1] 
In 2012 a riprap installation was built at Pier-3 to mitigate the local scour. The average size of the rocks used in the riprap was 1 Ton and it was estimated for conditions during a 100 year flood. Figure 5.3 shows the designed extent of the riprap around the affected pier overlaid on the top of the aerial view of the bridge.

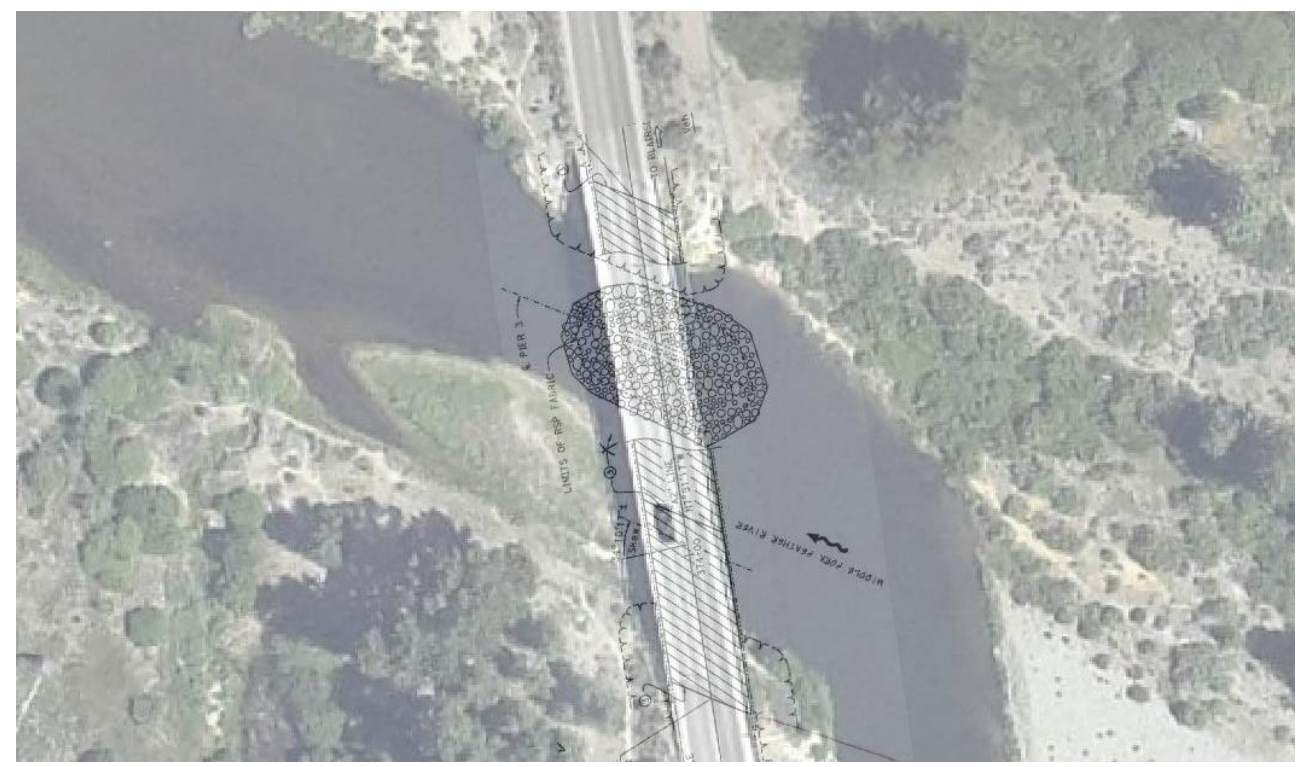

Figure 5·3: Designed rock layout for the pier protection [1]

A follow-up riverbed survey was performed in 2013. Reconstructed bathymetry based on this survey is shown in Figure 5.4. The scour hole was completely level with the surrounding bed. Figure 5.5 shows the work area near Pier-3 during the riprap installation in August of 2012.

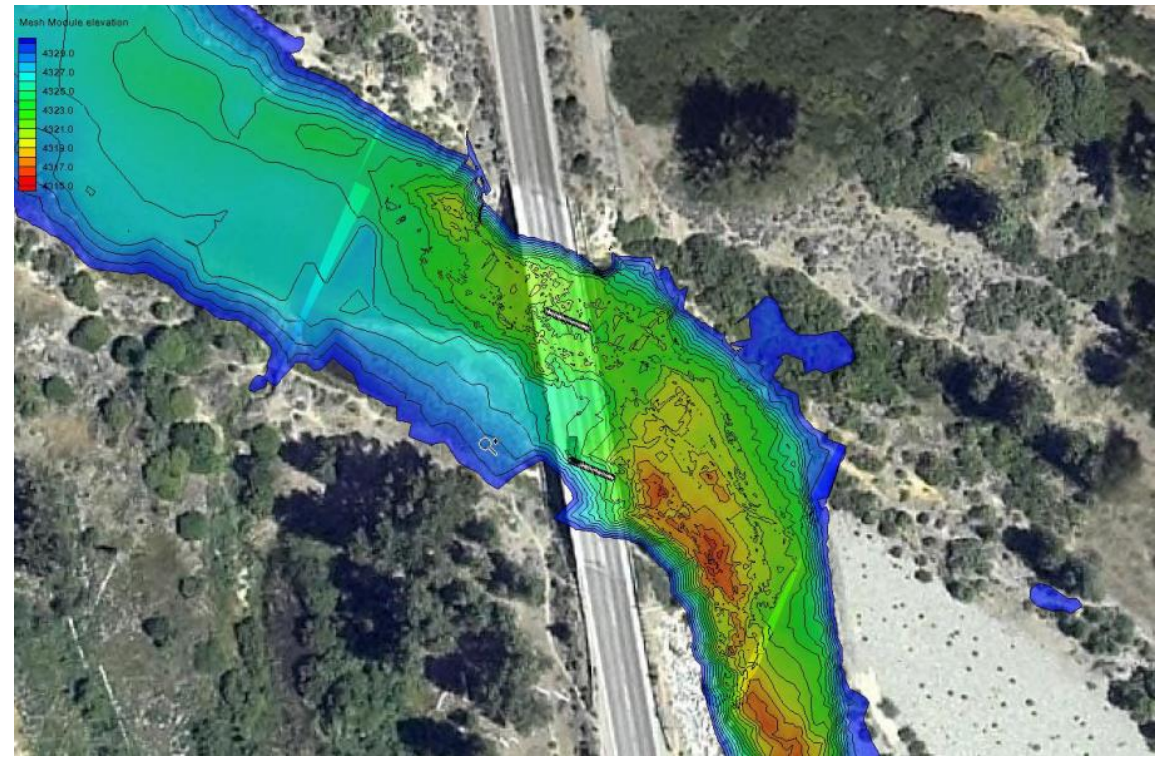

Figure 5.4: 2013 Bathymetry of the river after the riprap installation (2013) [1] 


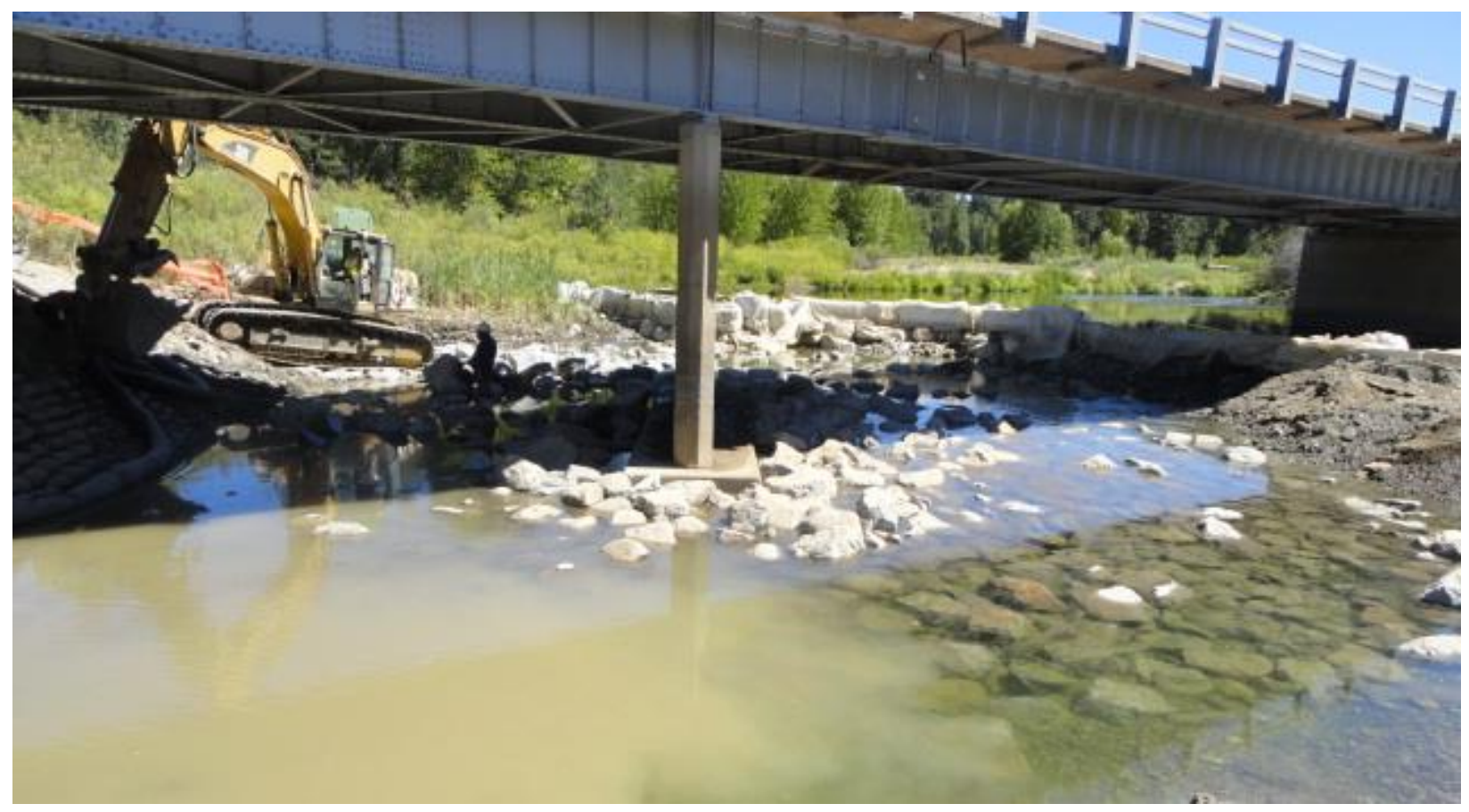

Figure 5.5: Riprap installation near Pier-3 in August of 2012 [1]

According to further investigation [1] the design of the riprap used a lower discharge than estimated later and did not account for the higher than anticipated velocities, associated with the bridge pier blockage of the flow. Therefore, there was concern that the riprap may fail during an extreme event like a 100 year flood. Advanced 3D FSI analysis was further recommended to investigate possibility of riprap failure during a 100 year flood event. 


\subsection{CFD Model Development}

The riverbed geometry model was constructed based on the 2011 bathymetry survey and GPS coordinates of the areas around the river. The bed surface was subdivided into the riverbed and grass portions so different roughness coefficients could be used for each of these boundaries in the CFD model (see Figure 5.6). Additionally, an area surrounding the pier was bounded with a box where denser mesh could be used for more accurate results.

The point cloud bathymetry was numerically enhanced using Matlab and MeshLab [5] and triangulated to obtain a raw surface. It was subsequently imported into STAR-CCM+ as the initial geometry of the numerical model. Then, the bed geometry together with the bridge geometry were transformed into a surface mesh, and a volume mesh was generated with polyhedral cells. The covered domain was approximately $285 \mathrm{~m}$ long, $120 \mathrm{~m}$ wide and about $7 \mathrm{~m}$ high. The boundary types used in the model are also shown in Figure 5.6. Due to problems with enforcing pressure boundaries on the outlet, where large recirculation zones were developing, the domain was extended by another $300 \mathrm{~m}$. There was no detailed data regarding the profile of the river in that region so the extension was built based on the last cross section of the surveyed domain with a slope of $0.3 \%$.

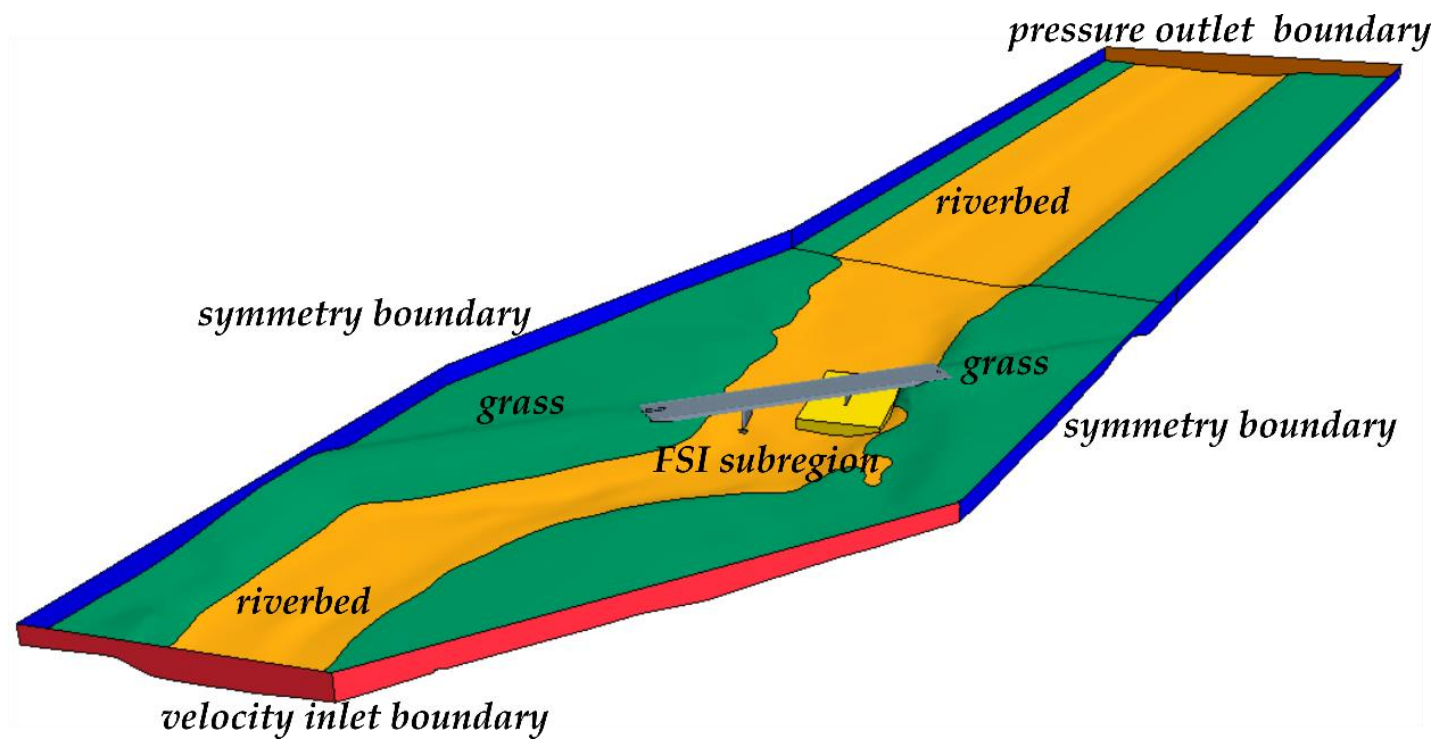

Figure 5.6: Definition of the bed boundaries in the CFD model

Figure 5.7 shows the surface mesh of the riverbed in the CFD model. Isosurfaces of the elevation are visible on the surface with the deepest point near Pier-3. 


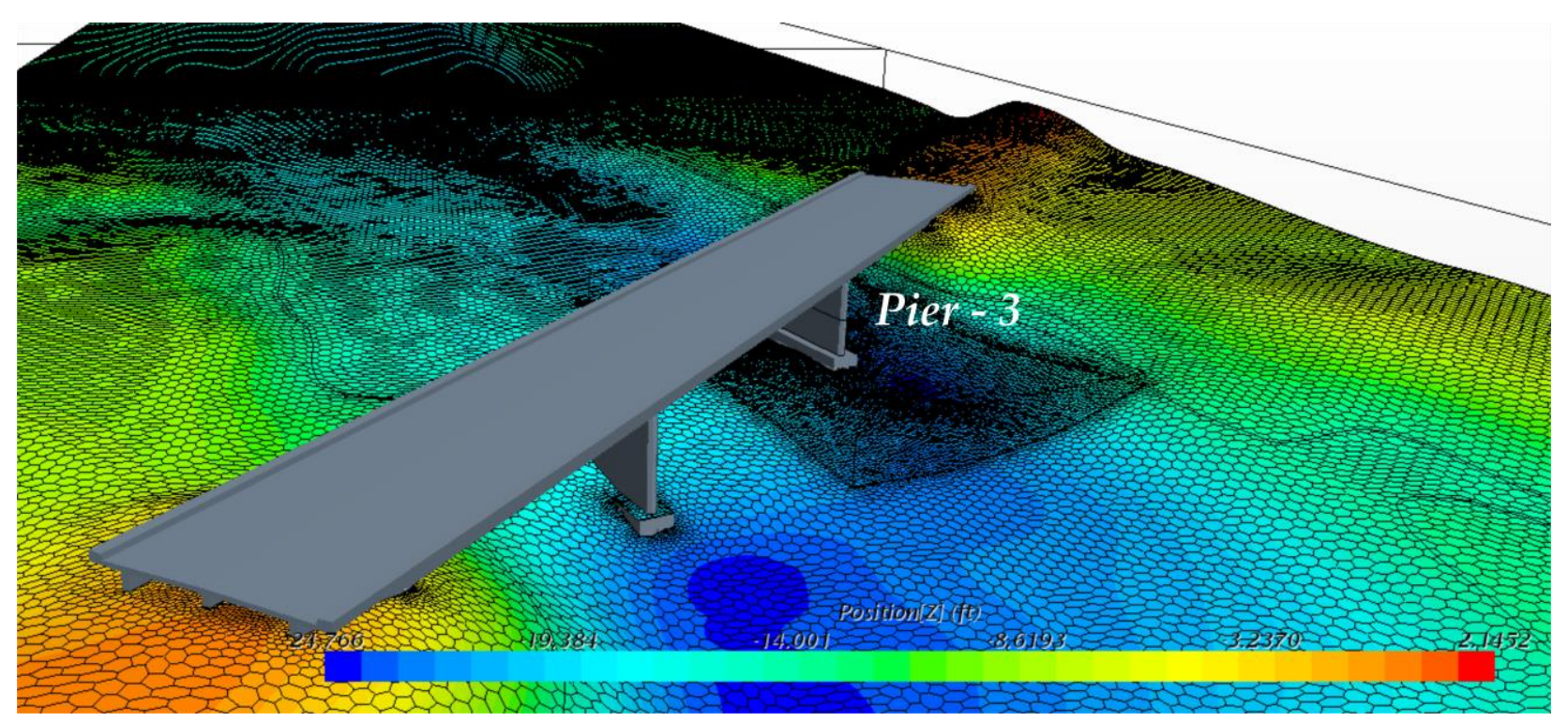

Figure 5.7: Surface mesh of the riverbed in CFD model

STAR-CCM+ software allows for fine gradation of the computational mesh in $3 \mathrm{D}$. In the areas of highest interest, very fine meshes can be specified. To save computational cost, coarse meshes can be used outside of areas of interest. Local refinements can be further defined based on control volumes, surfaces, or edges. Figure 5.8 shows the surface mesh of the bed after the rocks have been placed near the affected pier. The region in vicinity of Pier- 3 was separated from the rest of the domain. Internal interfaces were placed on the boundaries of that region that were in contact with the outer, global region. This was done not only to better control the mesh size, but also for the purpose of further FSI analysis. In the pure CFD analysis the entire domain together with the extensions was used. However, to simplify the FSI computations, only this sub region was used for coupled FSI analysis. The analyzed time in FSI computations was short (several seconds) as compared to the CFD analysis (several minutes). The water flow around the pier doesn't change in such short period of time. Thus, FSI analysis on the extracted subregion with appropriate boundary conditions copied from the interfaces used in CFD was considered to be equivalent to the global domain analysis. This approach not only reduces the computational time required by the model, through the reduction of the cell count but also removes the requirement to use multi-phase physics for the water-air interface reconstruction. Analysis of the subdomain uses the so called closed lid simulation in which the entire analyzed subdomain is submerged under water. Figure 5.8 shows a cross section through the volume mesh in the global model. The section marked with purple color is the subregion used in the FSI calculation. Figure 5.9 shows a close up view of the mesh in the subregion. Mesh in that region is significantly denser than in the rest of the global model. The subregion contained approximately $2.2 \mathrm{M}$ cells, while the global model had nearly $4.1 \mathrm{M}$ cells. 


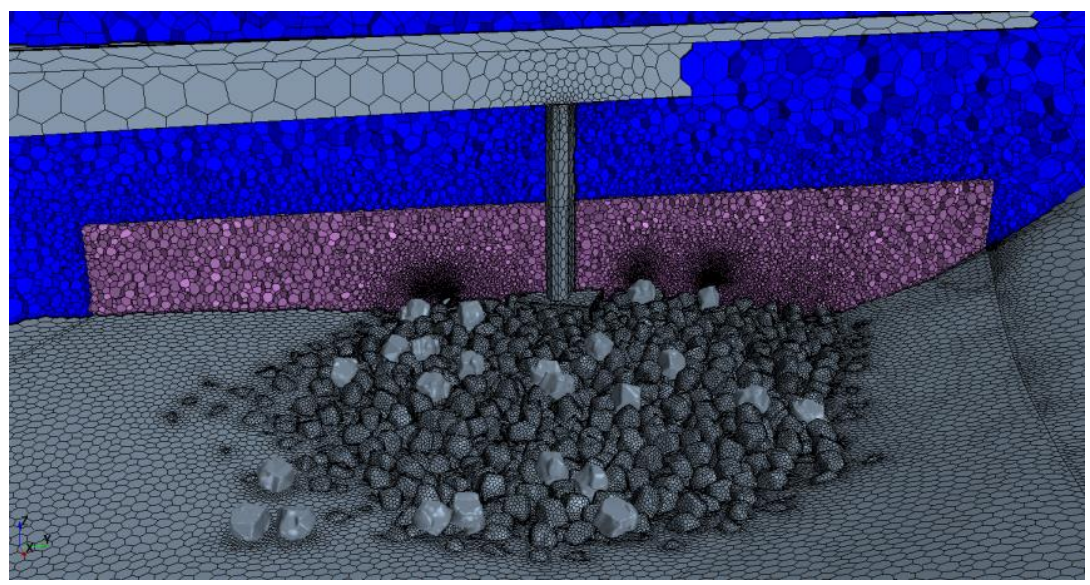

Figure 5.8: Cross section through the finite volume mesh used in STAR-CCM+ model

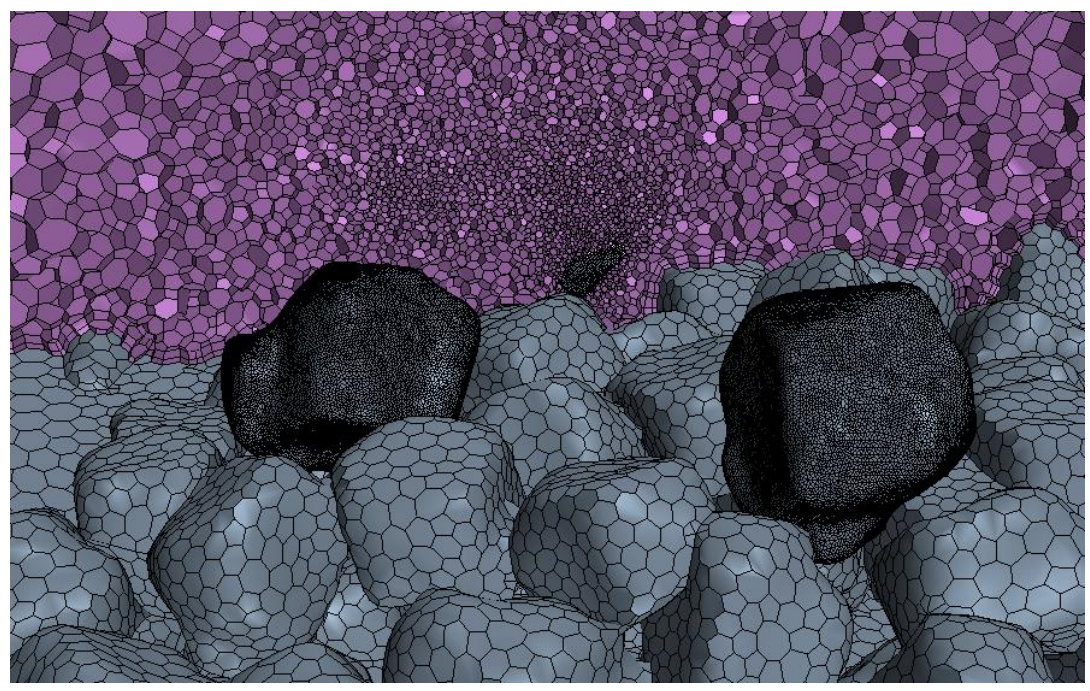

Figure 5.9: Cross section through the finite volume of subregion used in FSI computations.

\subsection{Comparison of Flow Conditions with 2D Analysis}

Before the FSI analysis was performed, the results of 3D CFD analysis were compared against results of 2D analysis performed by the California Department of Transportation (Caltrans). The 2D analysis was conducted using software called TUFLOW. One hundred year flood conditions were used for the comparative analysis in which the discharge of water at the inlet was 30,100 cfs. The details of those calculations can be found in [1]. The downstream water surface elevation (WSEL) for the 2D model was computed to be 4336.8 feet based on a normal depth calculation for the downstream cross-section using a slope of $0.3 \%$. This WSEL probably has 2 feet of uncertainty due to the lack of calibration data. The water surface upstream of the bridge is approximately 4341 feet and flows under pressure with the bottom of the bridge girders at 4338.9 feet. These elevations give about $24 \mathrm{ft}$ water depth upstream and about $14 \mathrm{ft}$ water depth downstream of the bridge. The depth averaged flow velocity upstream of the bridge is roughly 11 $\mathrm{ft} / \mathrm{s}$ with peak velocities reaching about $15.5 \mathrm{ft} / \mathrm{s}$ downstream from the bridge. 


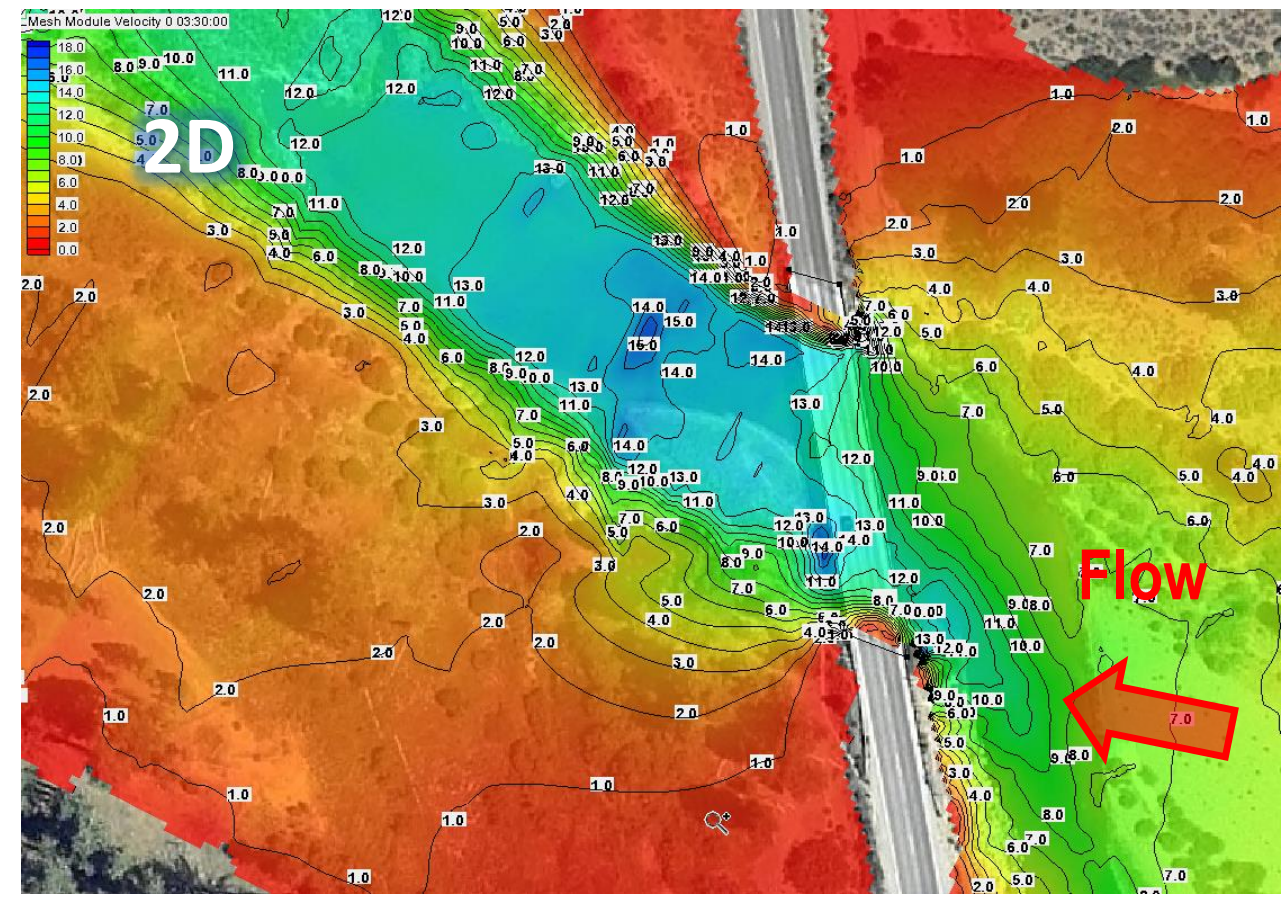

Figure 5.10: Depth averaged velocities in the 2D TUFLOW calculation

Three dimensional CFD calculations were performed with a time step of $0.1 \mathrm{sec}$. Pseudo steady state conditions were achieved in about 700 seconds of simulated time. It is not trivial to extract depth averaged velocities (and other quantities as well) from the $3 \mathrm{D}$ calculation. For that reason, surface velocity was extracted and the depth averaged velocity was only calculated in a cross section under the bridge for which the data from $2 \mathrm{D}$ analysis was also available. Figure 5.11 shows the water velocity at the surface as calculated in STAR-CCM+ for the 100 year flood case. The velocity is noticeably higher than the depth averaged velocity from the $2 \mathrm{D}$ analysis. The water depths estimated in 3D were $18 \mathrm{ft}$ upstream and $11.5 \mathrm{ft}$ downstream of the bridge as compared to $24 \mathrm{ft}$ and $14 \mathrm{ft}$ respectively in 2D analysis. The increased velocity is a consequence of the reduced WSEL. 2D analysis predicted for the 100 year event overtopping of the bridge, while the 3D analysis predicted that the water level will reach the girder level. Figure 5.12 and Figure 5.13 show the water surface level near the bridge together with velocity magnitude and relative height of the water surface level. 


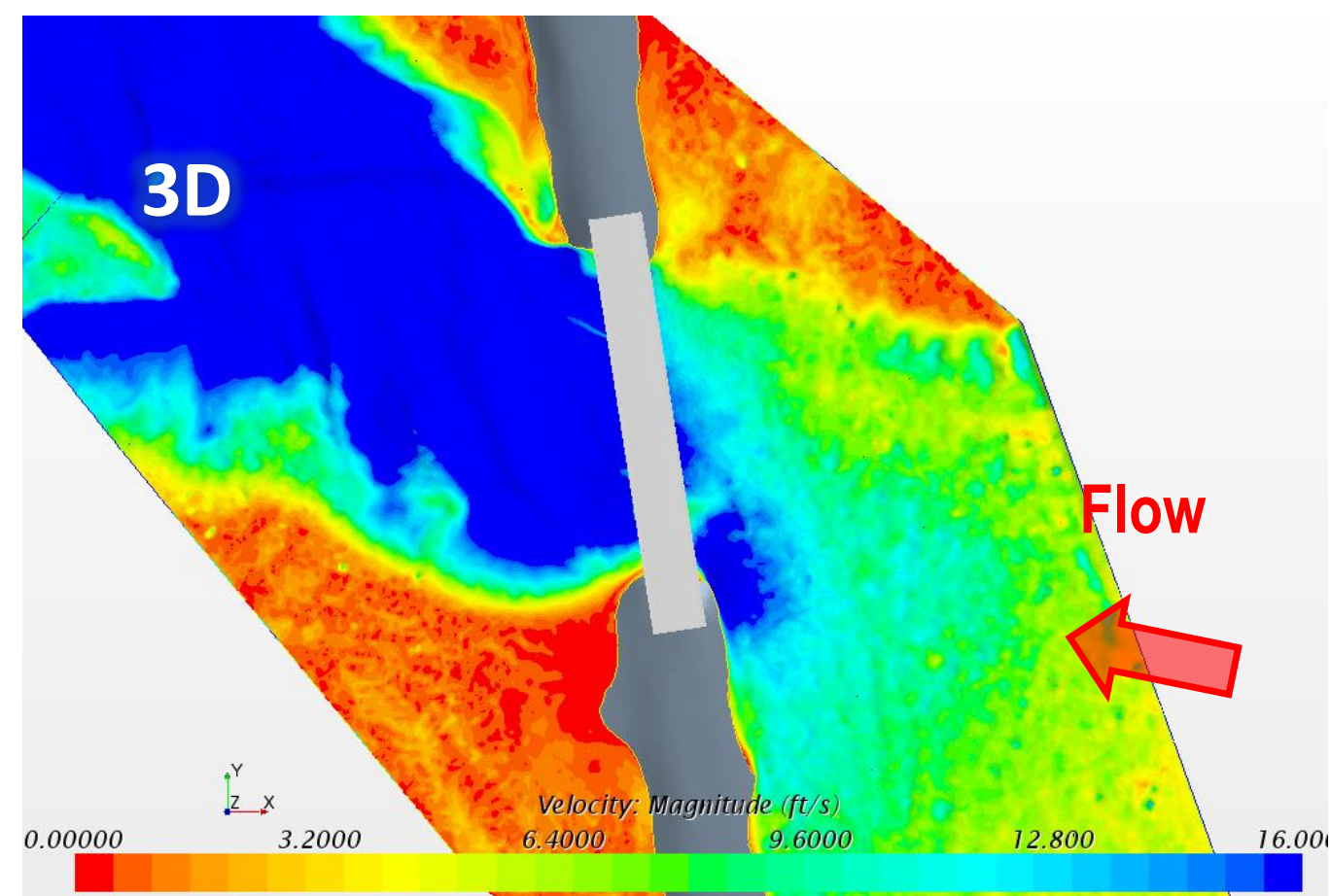

Figure 5.11: Water surface velocity in 3D calculation with Star-CCM+

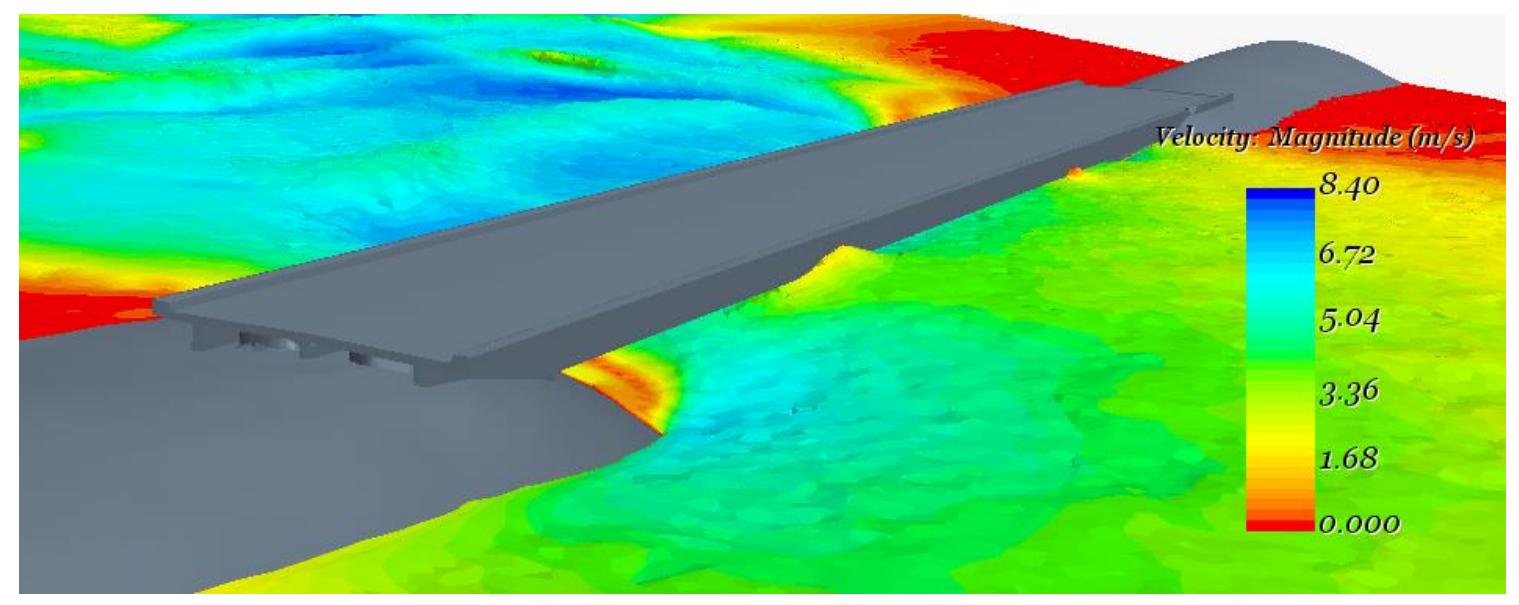

Figure 5.12: Perspective view of the water surface near the bridge with overlaid velocity

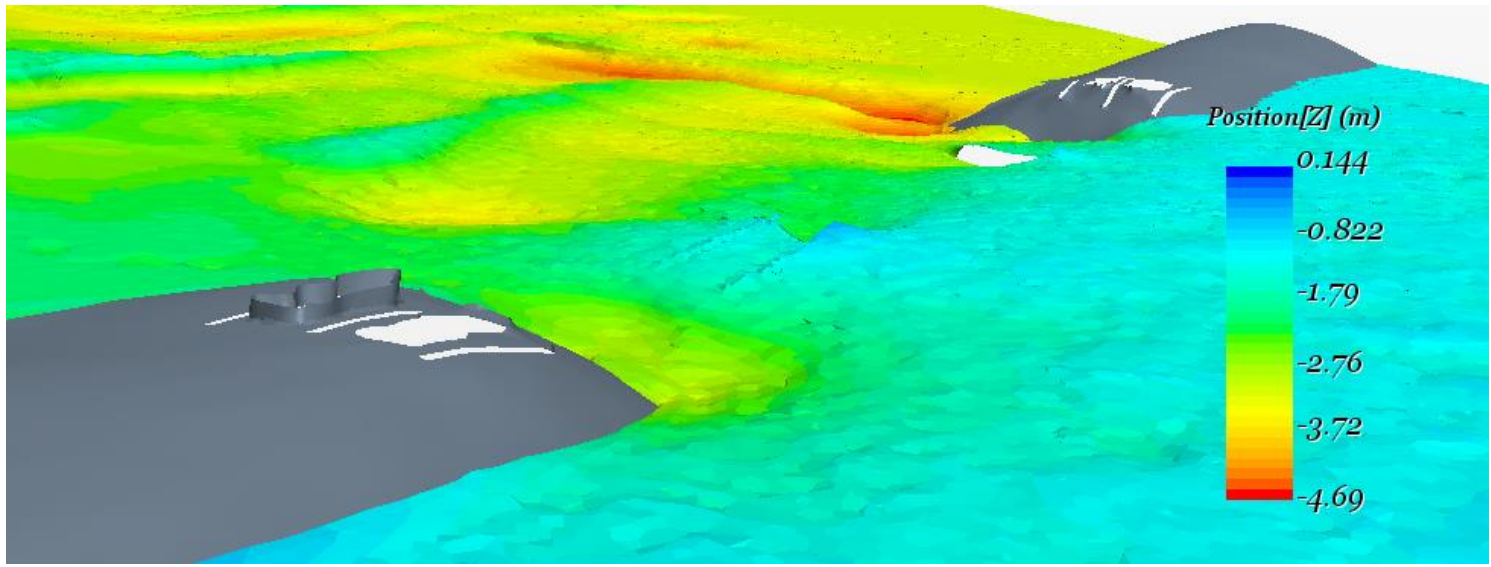

Figure 5.13: Perspective view of the water surface level under the bridge with overlaid relative height 
Figure 5.14 compares the depth averaged velocity obtained in $2 \mathrm{D}$ and $3 \mathrm{D}$ analysis for a cross section under the bridge. The velocities are in agreement only on the outer side of the affected pier. However, for the rest of the cross section, the velocity obtained in $3 \mathrm{D}$ analysis was significantly higher (around $17.5 \mathrm{~m} / \mathrm{s}$ for $3 \mathrm{D}$ analysis as compared to around $13.5 \mathrm{~m} / \mathrm{s}$ for $2 \mathrm{D}$ analysis). HEC23 provides a design guideline for sizing of the rock based on the design velocity at the pier. Using this equation 1 Ton rock was calculated for the countermeasure installed in 2012. This procedure, however, does not address the combined effects of the scour potential caused by both the water acceleration around the pier blockage and pressure flow. Updated analysis [1] indicated that this combined effect may cause the riprap to fail during a 100 year event.

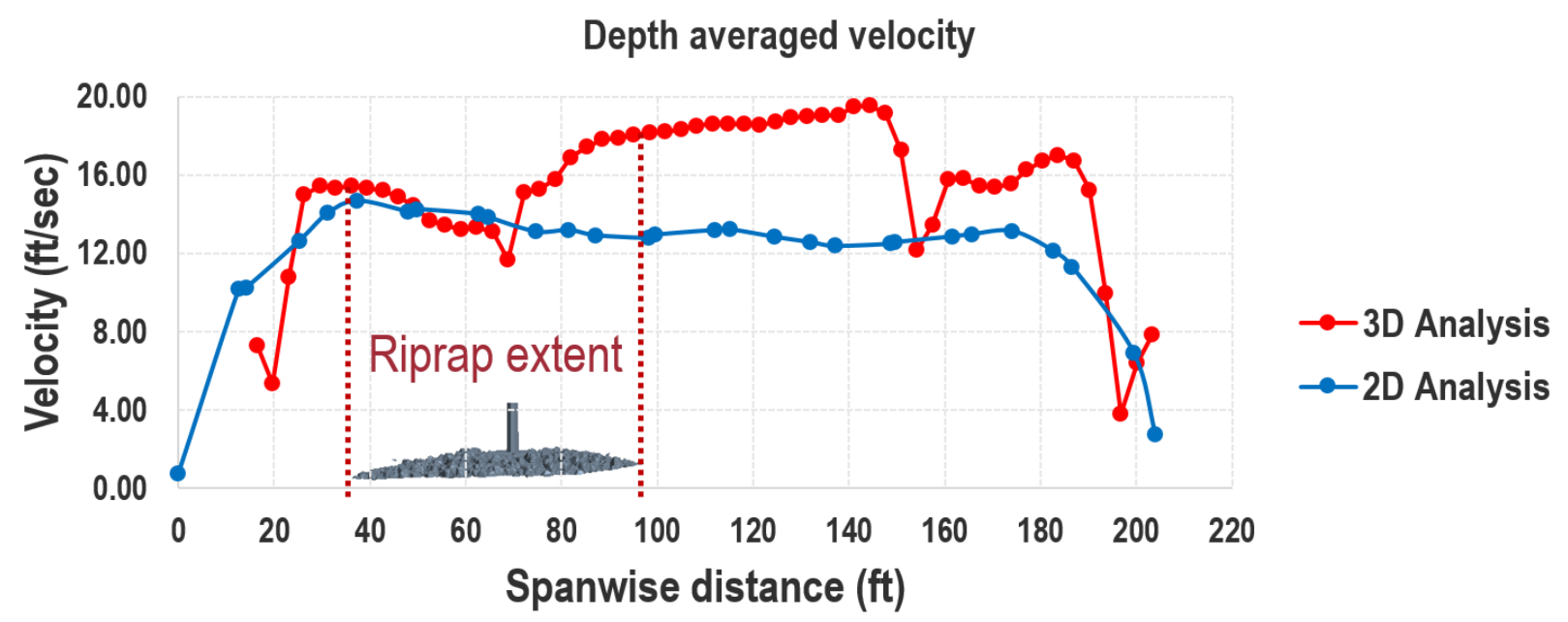

Figure 5.14: Depth averaged velocity under the bridge in 2D and 3D calculations

\subsection{Riprap Geometry Development}

For the initial CFD models the riprap extent and its surface slopes were reconstructed using the design drawings. Figure 5.15 shows the cross sections of riprap installation from that documentation. Over 2,500 rocks were included in the CFD model. They were placed in the scour hole semi-manually, meaning smaller clusters of rocks were copied and changed slightly to introduce some variation in their shape and layouts. After filling the entire scour hole with the rocks, the surface wrapper was used in STAR-CCM+ to smooth out the geometry and create one continuous bed boundary. The final triangulated surface of the river bed is shown in Figure 5.16. Just like in the validation test simulation, the geometry of the rocks required several simplifications in order to make the cost and effort for geometry construction reasonable. The shape and the layout of the rocks are much less random than they are in reality. Void spaces between the rocks in the riprap may be bigger allowing for more flow within the riprap. To better understand these effects, TFHRC ordered another, more detailed investigation of the installed riprap using sonar techniques. Together with the pictures of the riprap taken during low flows they aim to complement the numerical modeling and improve its accuracy. It should be noted that from the pictures it appears that many rocks have dimensions indicating their mass is less than 1 Ton. For that reason in the simulations rocks with the mass between 0.4 and 1 Ton were used. 

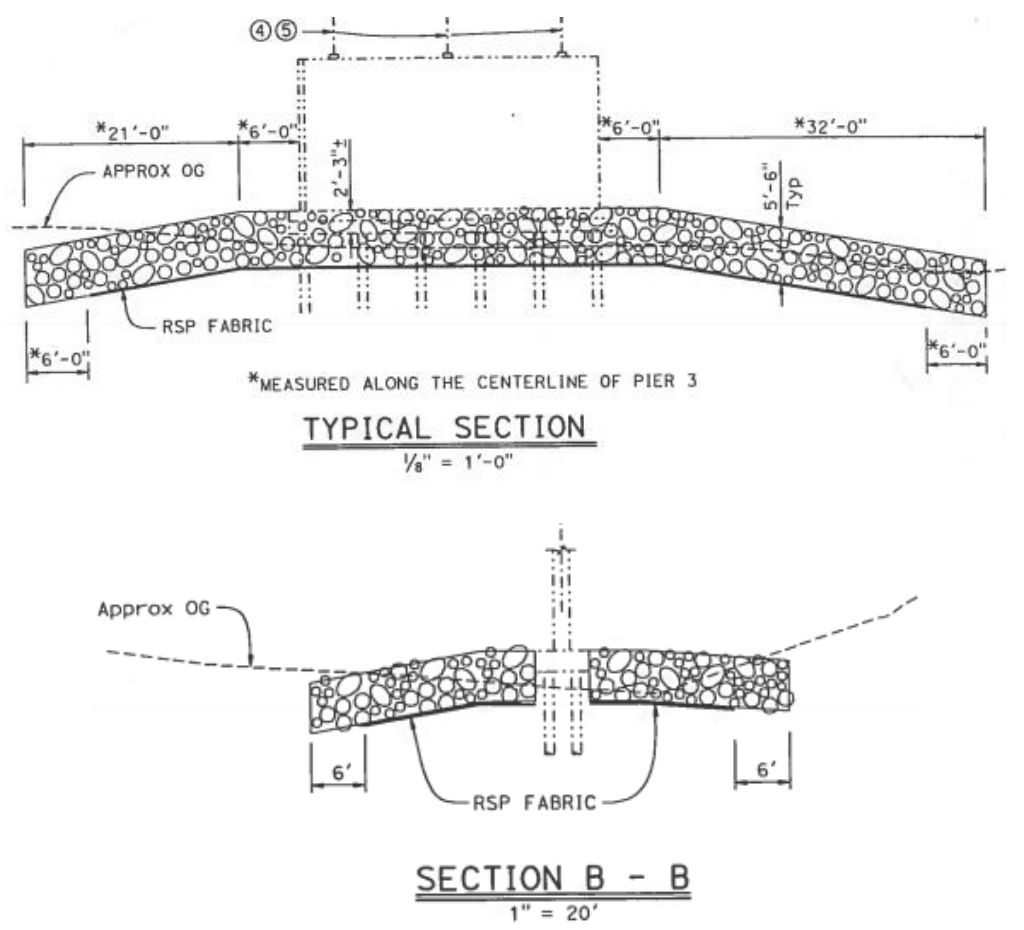

Figure 5.15: Design drawings of the riprap around Pier-3 of the bridge
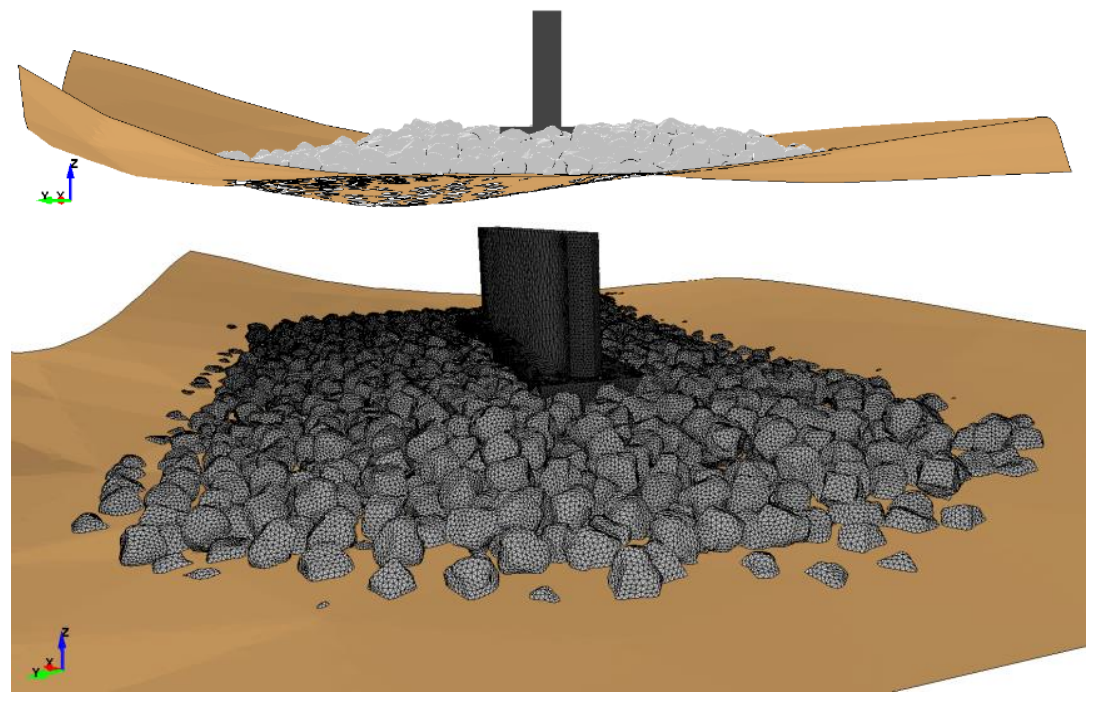

Figure 5.16: Geometry of the riprap used in the CFD model 

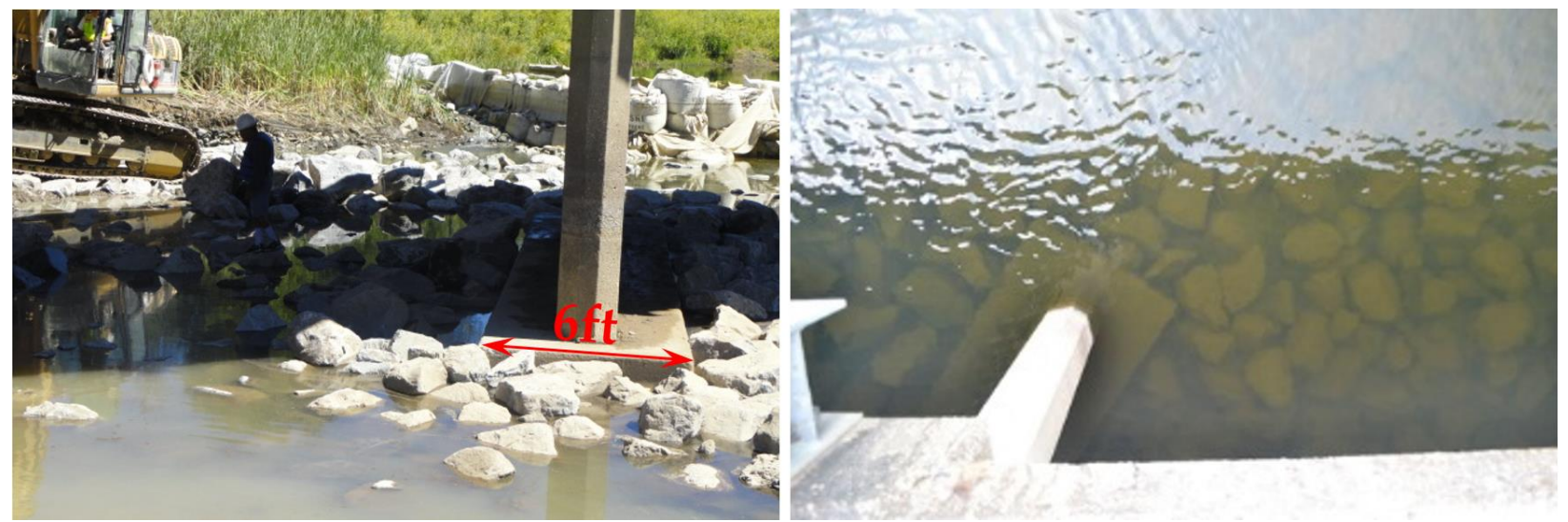

Figure 5.17: Pictures of riprap installation near Pier-3 during and after the construction

TFHRC contracted three different companies to perform sonar scanning of the bed: Blueview, CodaOctopus, and Fenstermaker to assess the quality and usefulness of the data provided by them. Each of these companies uses different techniques for scanning the underwater profiles. The initial goal was to be able to reconstruct the bed surface based on the scans in order to maintain high level of realism in CFD models. However, none of the techniques used allowed for this. The sonars are not physically able to scan voids between the rocks precisely because most of them are out of the equipment view lines. The geometries created based on these limited scans would reduce to a minimum the flow between the rocks by closing voids in the rock layers that do not show up in scans. Thus, the primary use of these scans was to define the overall geometry of the riprap installation in terms of its extent and to estimate the correct size of the rocks. The scans allowed for identifying the precise extent of the riprap and slopes on each side of the pier. The shape of the new embankment was also easily identifiable. Figure 5.18 and Figure 5.19 show a point cloud of the scanned bed by BlueView. The scans reveal that the borders of the riprap differ slightly from the designed shape. The side embankment was also not present in the design drawings available when the CFD model was constructed. Thus, it also had to be accounted for the in the model. 


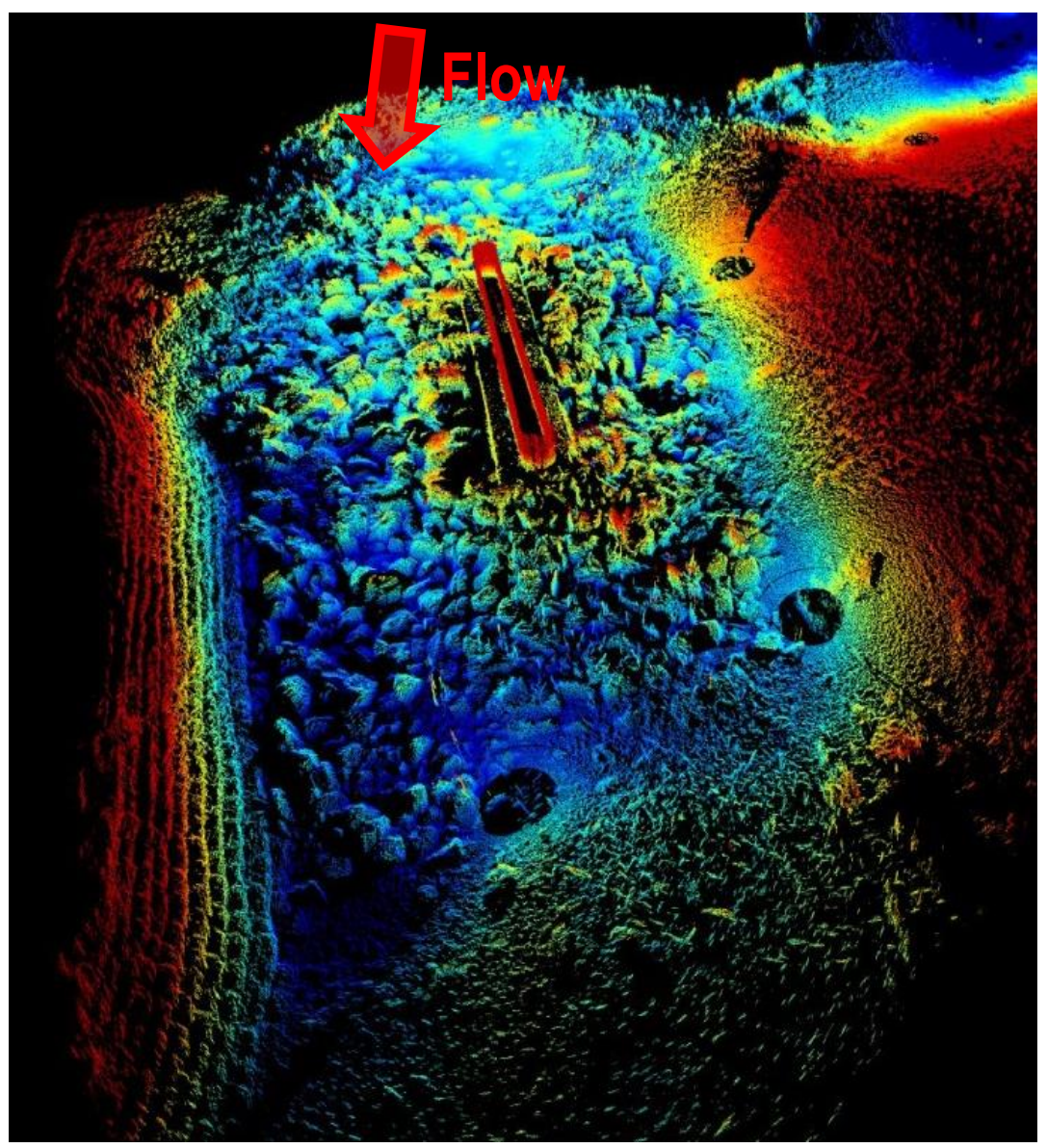

Figure 5.18: Top view on the installed riprap

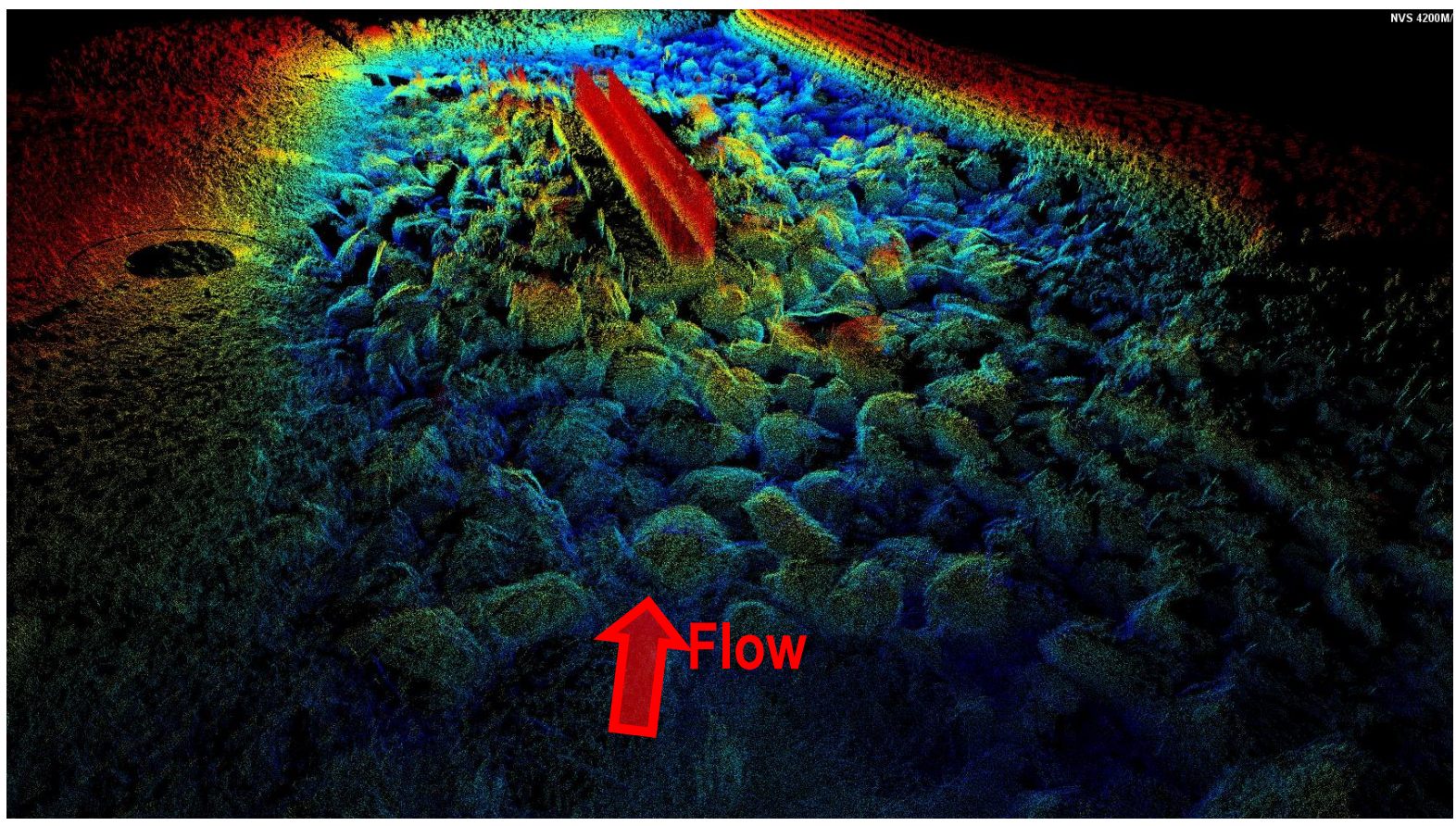

Figure 5.19: Front view on the pier with installed riprap 


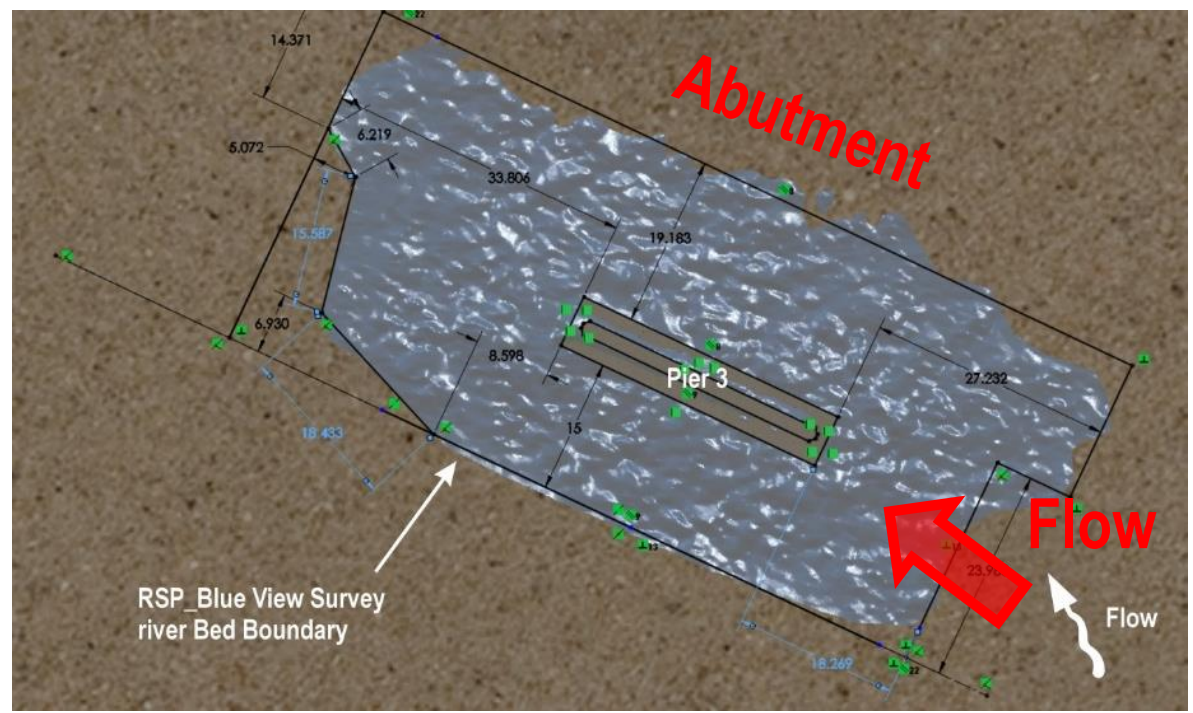

Figure 5.20: The extent of the riprap derived from the bed scan.

Figure 5.20 shows the extent of the boundaries of the scanned riprap. These boundaries were placed over the top view of the riprap in the CFD model and shown in Figure 5.21. There are several areas where the boundaries do not match and the bed geometry was adjusted, including the addition of the embankment on the right-hand side of the Pier-3. Figure 5.22 shows the updated geometry of the river bed near Pier-3. The new geometry matches the extent of riprap from the survey much more closely.

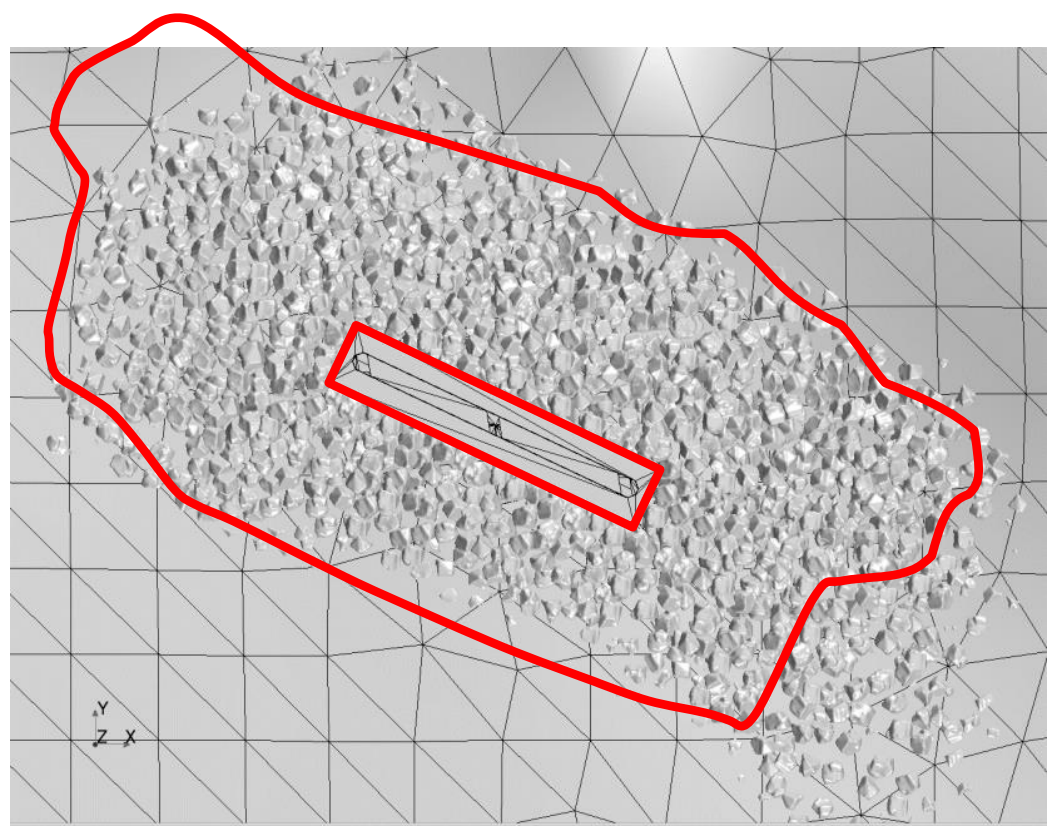

Figure 5.21: Extent of the riprap in the original CFD model 


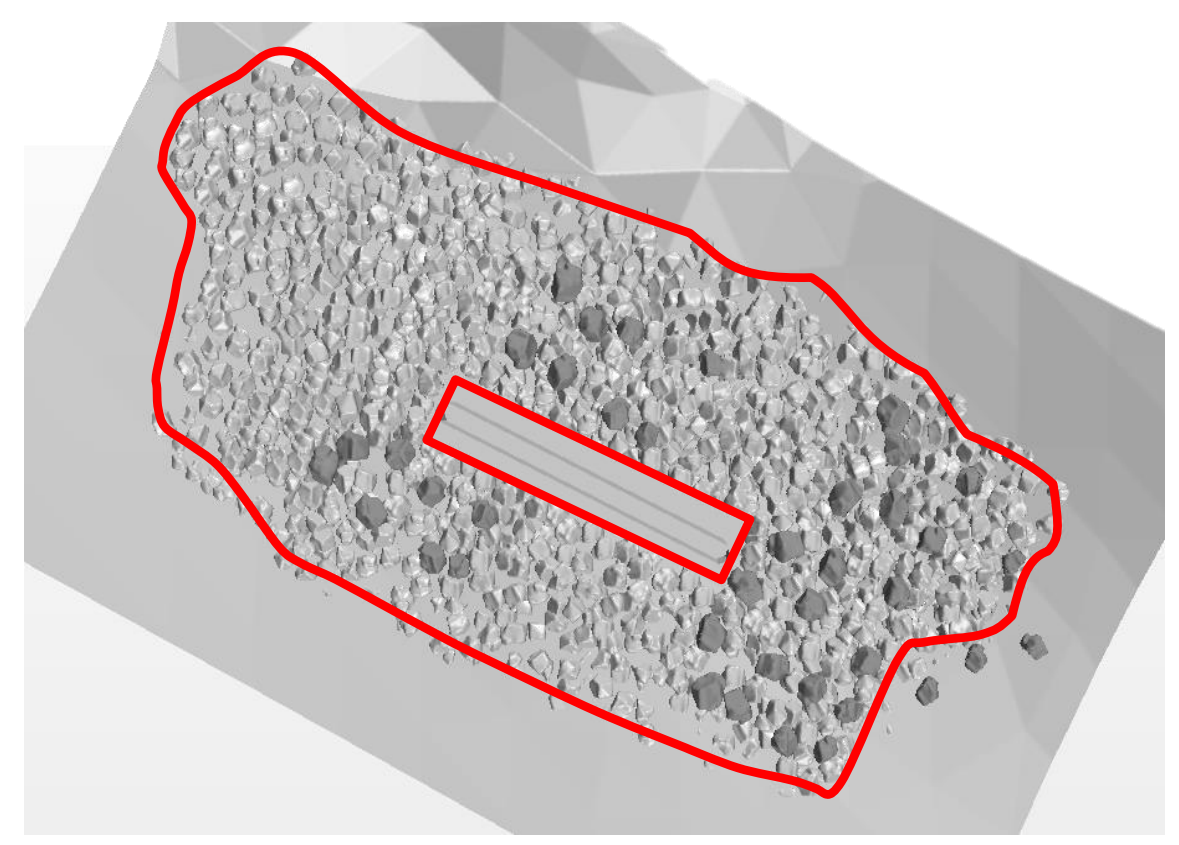

Figure 5.22: Extent of the riprap in the updated CFD model with movable rocks

The placement of the movable rocks for onset of motion analysis is shown in Figure 5.22. They are displayed with a darker grey color. As mentioned earlier it could be clearly seen from the pictures of the Pier-3 site that many of the rocks couldn't be 1 ton rocks. One ton rocks should be close to 3 foot diameter spheres (or equivalent). With the pier foundation being $6 \mathrm{ft}$ wide, these rocks should be about half of the footing width or more. Figure 5.17 and Figure 5.19 show that few such rocks can be found and many of them are significantly smaller than $3 \mathrm{ft}$ in hydraulic diameter. For that reason it was assumed that in the FSI simulation 40 rocks of different sizes would be included: ten $1 \mathrm{~T}$ rocks, ten $0.8 \mathrm{~T}$ rocks, ten $0.6 \mathrm{~T}$ rocks, and ten $0.4 \mathrm{~T}$ rocks.

\subsection{Finite Element Method Model Development}

The development of the finite element model followed the same procedure as explained in Chapter 4 for the validation case. The surface of the rocks after wrapping was extracted and used in LS-DYNA as a rigid boundary. Before the extraction an approximate location of movable rocks was decided upon. Subsequently the movable rocks were dropped into position in the LS-DYNA simulation and their final position is semi-random. The simulated drop assures that the rocks are in an equilibrium position before the fluid structure interaction, FSI, simulation starts. Figure 5.23 shows the vicinity of pier-3 together with moveable rocks in their final positions (pre-FSI). The $0.4 \mathrm{~T}$ rocks are highlighted with orange color, $0.6 \mathrm{~T}$ rocks are green, $0.8 \mathrm{~T}$ rocks are red, and 1.o T rocks are blue. Some of the moveable rocks are sticking slightly above the neighboring nonmoveable rocks. The 0.4 Ton rocks in the front of the riprap are entirely exposed to the flow. This is another factor contributing to conservatism in the computed results. 


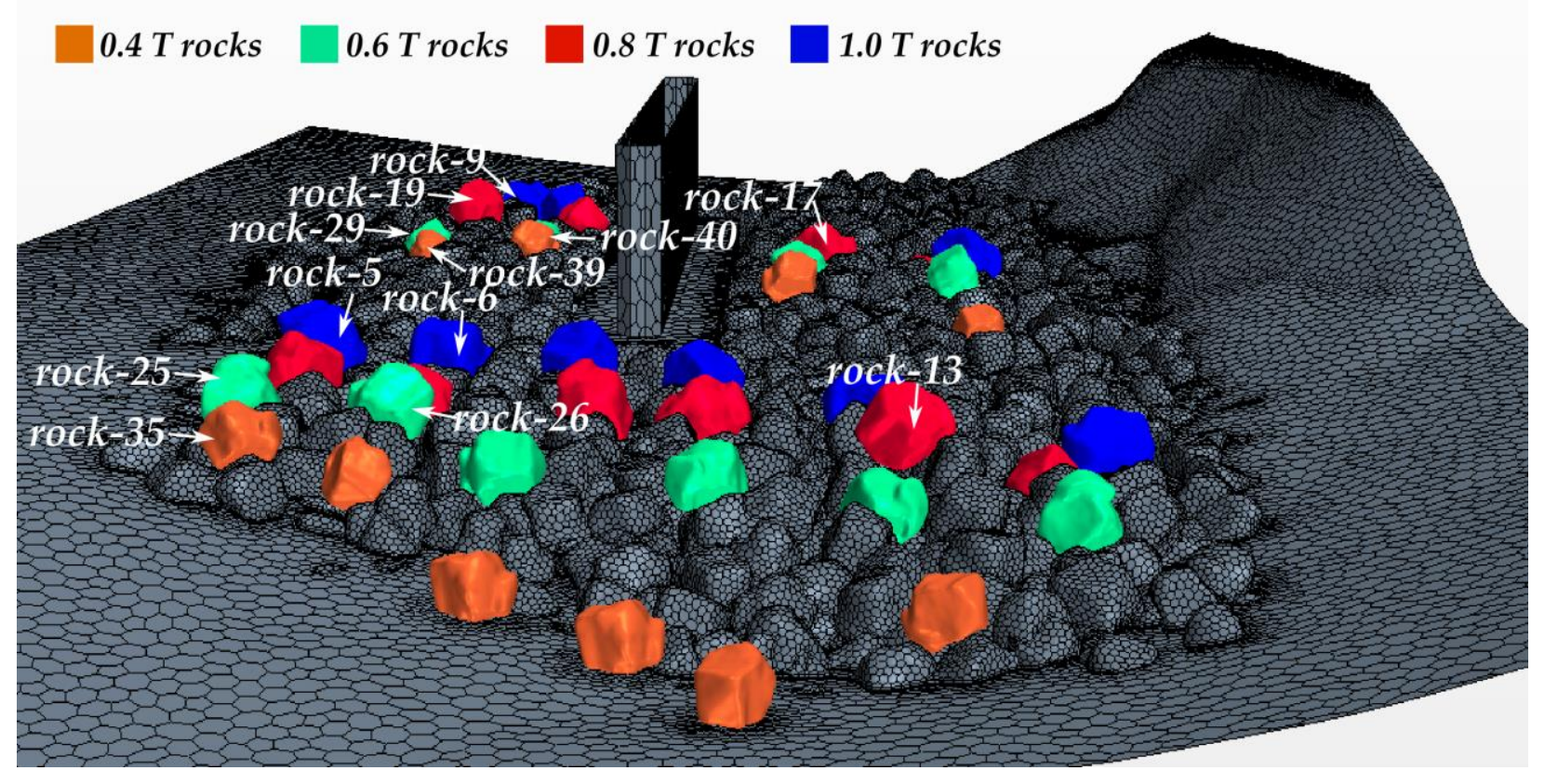

Figure 5.23: Placement of moveable rocks around the pier.

\subsection{Analysis and Results}

\subsubsection{Results of CFD Analysis}

From the initial CFD analysis, hydrodynamic forces on all moveable rocks were extracted in a local coordinate system that was aligned with Pier-3 and the flow under the bridge. The histories of the $\mathrm{Z}$ (vertical) and $\mathrm{Y}$ (stream wise) components of these forces are shown in Figure 5.24. The $X$ (cross stream) components weren't significant. The highest $Y$ components of the forces reached about $2.5 \mathrm{kN}$, and highest $\mathrm{Z}$ components reached about $5.5 \mathrm{kN}$. Figure 5.23 labels also the rocks with highest forces as found in the CFD simulation of a 100 year flood event. The three most critical rocks in each weight category are labeled. Most of the critical rocks are located west from the pier closer to the center of the bridge opening, where the average velocity was the highest. Table 5.1 shows all the force components on these twelve most vulnerable rocks. Although these are the rocks with the highest forces acting on them, the final outcome of FSI suggests that some other rocks may move first. Their interaction with other rocks is the crucial and in many cases the deciding factor with respect to their stability in the riprap installation. From this analysis it appears that the most vulnerable in overall are the smallest rocks, as expected. The ratio between their weight and the resultant force acting on them is the highest. 


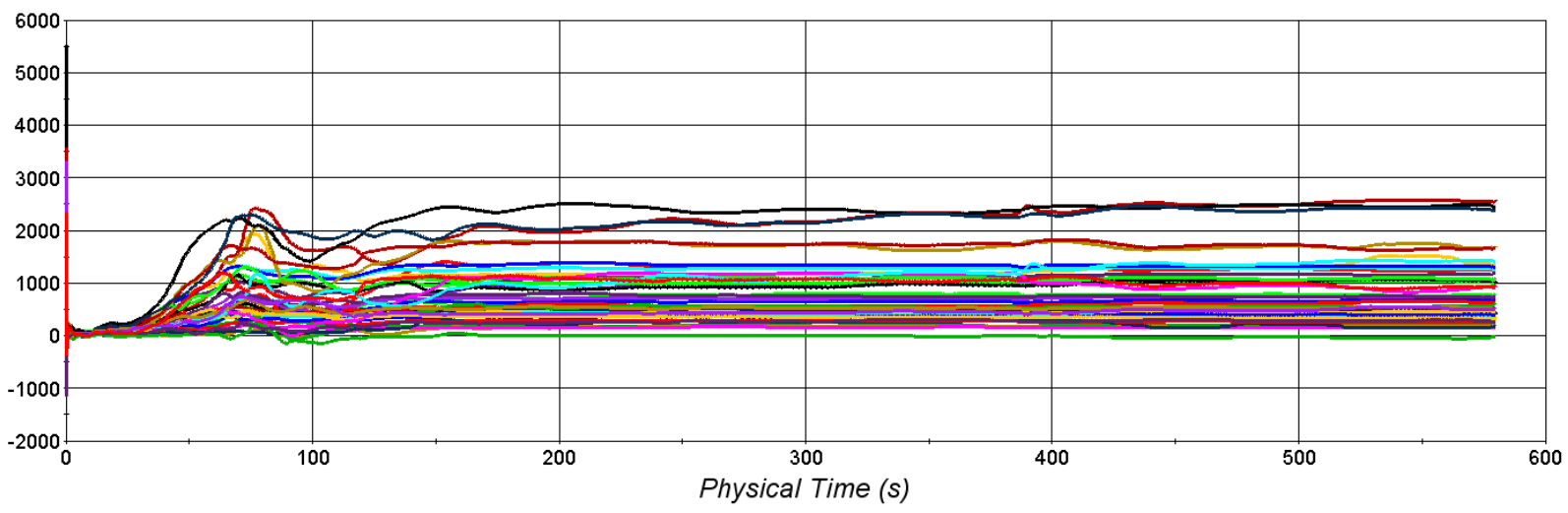

Z Forces

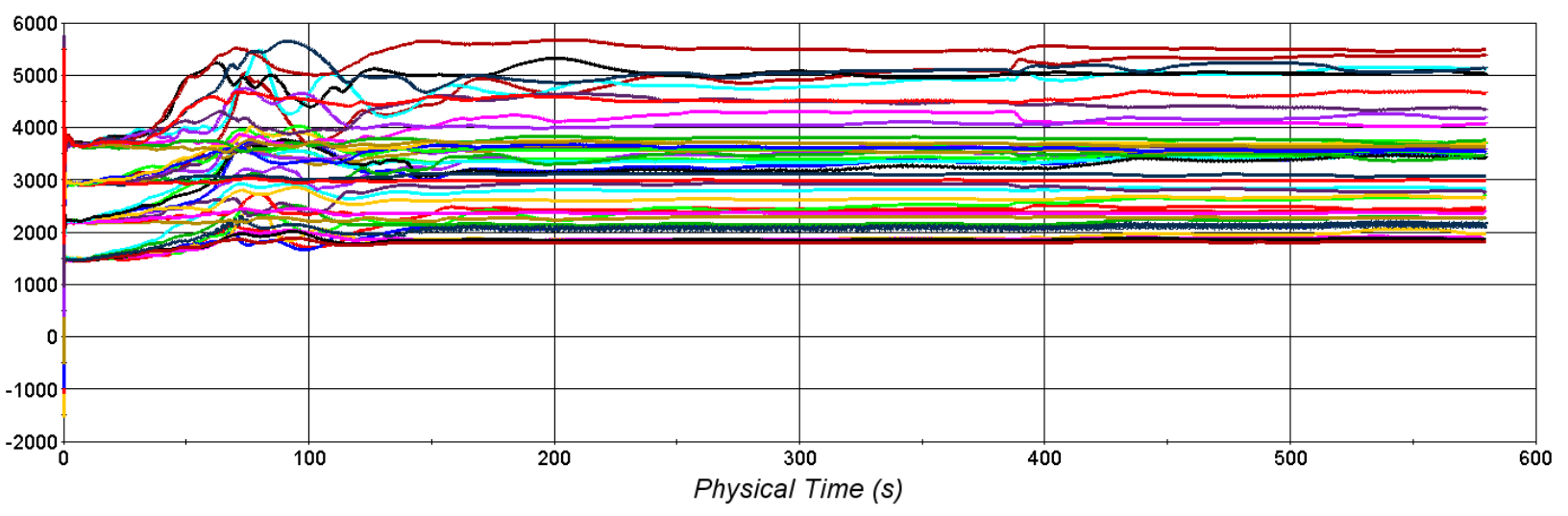

Figure 5.24: Forces on the moveable rocks in the simulation with discharge of 30,100 cfs (100 year flood) 
Table 5.1: Forces on the critical rocks in the simulation with discharge of 30,100 cfs (100 year flood)

\begin{tabular}{|c|c|c|c|c|}
\hline Rock \# & X Force & Y Force & Z Force & Resultant \\
\hline \multicolumn{5}{|c|}{1 Ton $(9.81 \mathrm{kN})$} \\
\hline 6 & -0.06 & 1.68 & 5.50 & 5.75 \\
\hline 5 & -0.87 & 2.45 & 5.10 & 5.72 \\
\hline 9 & -1.31 & 1.45 & 5.15 & 5.51 \\
\hline \multicolumn{5}{|c|}{$0.8 \operatorname{Ton}(7.85 \mathrm{kN})$} \\
\hline 19 & -0.15 & 2.50 & $5 \cdot 35$ & $5 \cdot 91$ \\
\hline 17 & -0.40 & 1.70 & 3.70 & 4.09 \\
\hline 13 & 0.09 & 1.10 & 3.75 & 3.91 \\
\hline \multicolumn{5}{|c|}{0.6 Ton $(5.88 \mathrm{kN})$} \\
\hline 25 & -0.95 & 1.32 & 3.45 & 3.81 \\
\hline 29 & -0.24 & 1.06 & 3.55 & 3.71 \\
\hline 26 & -0.38 & 1.08 & 3.45 & 3.64 \\
\hline \multicolumn{5}{|c|}{$0.4 \operatorname{ton}(3.92 \mathrm{kN})$} \\
\hline 35 & 0.43 & 1.25 & 2.80 & 3.10 \\
\hline 39 & 0.10 & 0.80 & 2.65 & 2.77 \\
\hline 40 & 0.10 & 1.20 & 2.45 & 2.73 \\
\hline
\end{tabular}

\subsubsection{Results of FSI Analysis}

In order to perform the FSI analysis, the problem was further simplified, as mentioned earlier. To conserve computer resources, the FSI analysis was restricted to a subdomain close to the pier with highest scour risk with the movable rocks on the riprap bed. This subdomain of interest together with velocity vectors on the outer boundaries is shown in Figure 5.25. Velocities in the plane just above the riprap rocks are shown in Figure 5.26. The average inlet velocity on the frontal face for that case was $11.1 \mathrm{ft} / \mathrm{s}$.

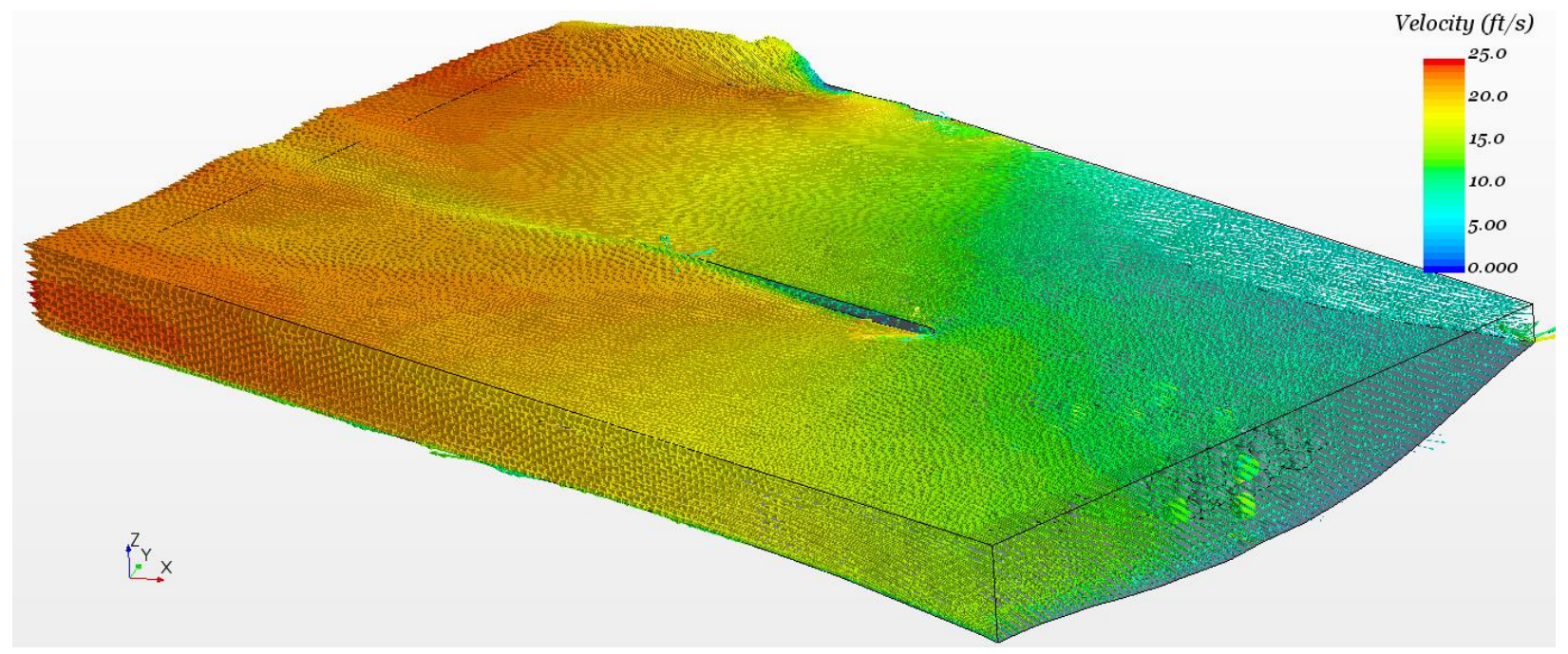

Figure 5.25: Velocity vectors on the interfaces between the scour subregion and the global domain 


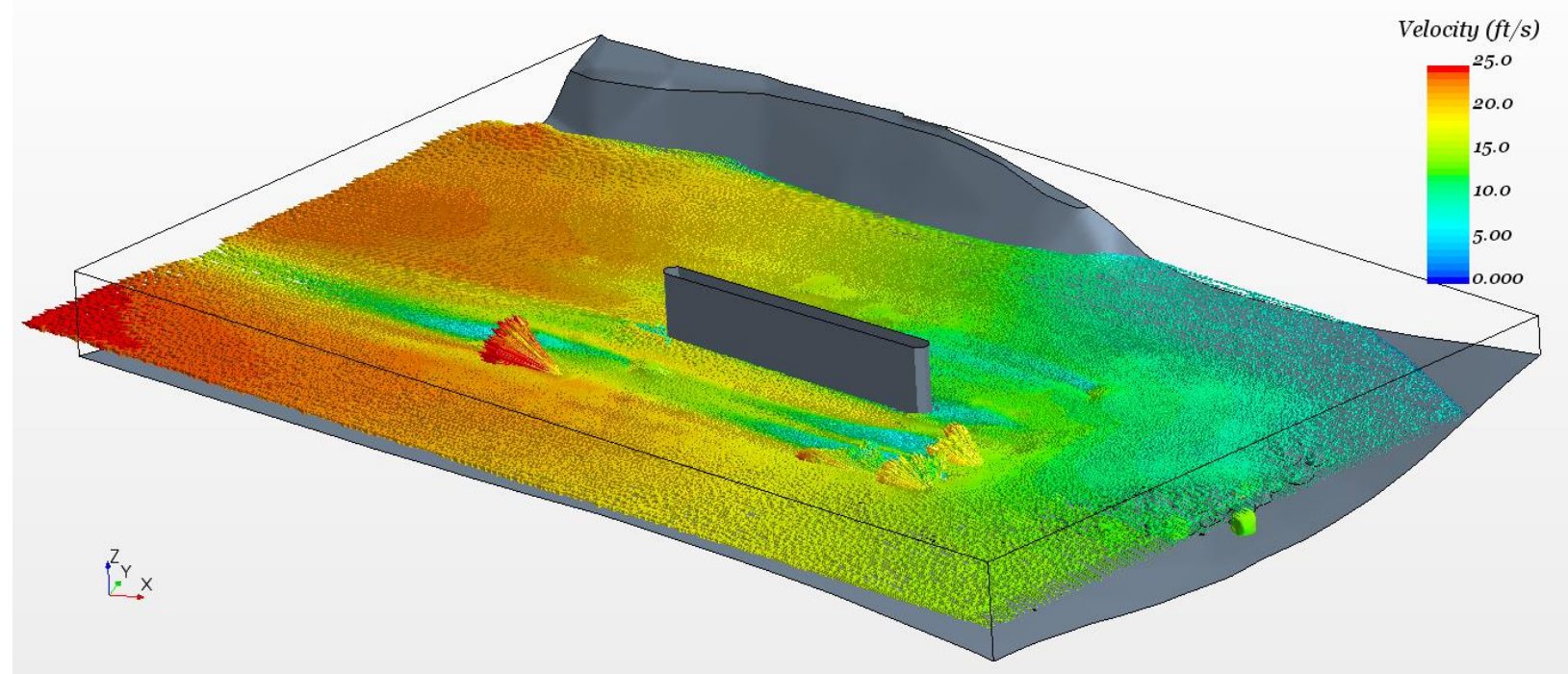

Figure 5.26: Velocity vectors on the plane just above the bed rocks

Figure 5.27 shows snapshots from the FSI simulation for the 100 year flood event. Four rocks have moved in that simulation. Two of them: rock 25 (o.6 T) and rock 19 (o.8T) were moved away from the riprap installation. Rock $5(1.0 \mathrm{~T})$ and rock $37(0.4 \mathrm{~T})$ only moved locally. These results indicate that 1.0 T rocks in that installation are safe although they might be close to onset of motion. Figure 5.28 additionally shows the trajectories of the center of gravity for the moving rocks. 


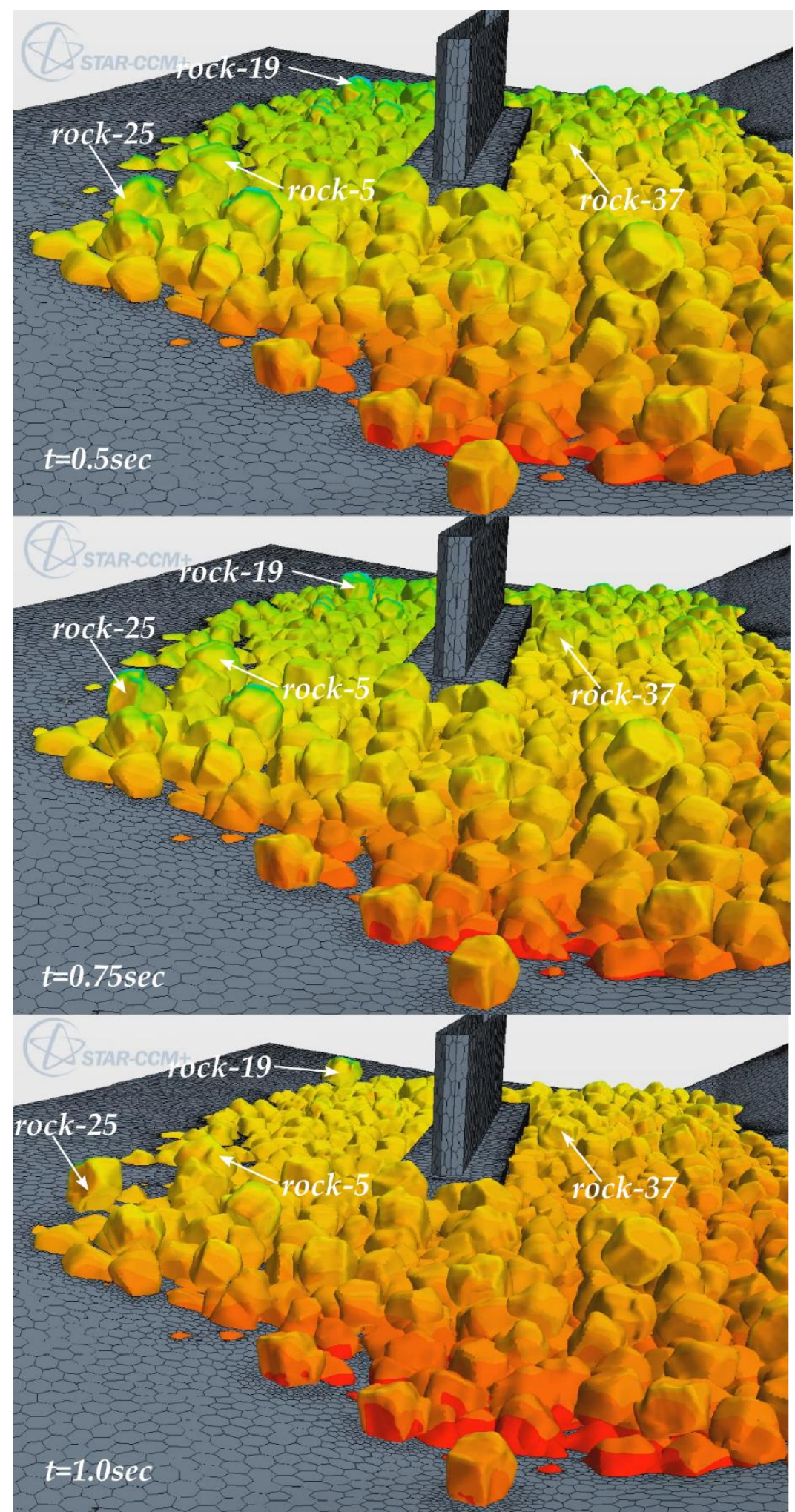

Figure 5.27: Snapshots of the FSI simulation for Q100 discharge (11.1 ft/s average inlet velocity) 


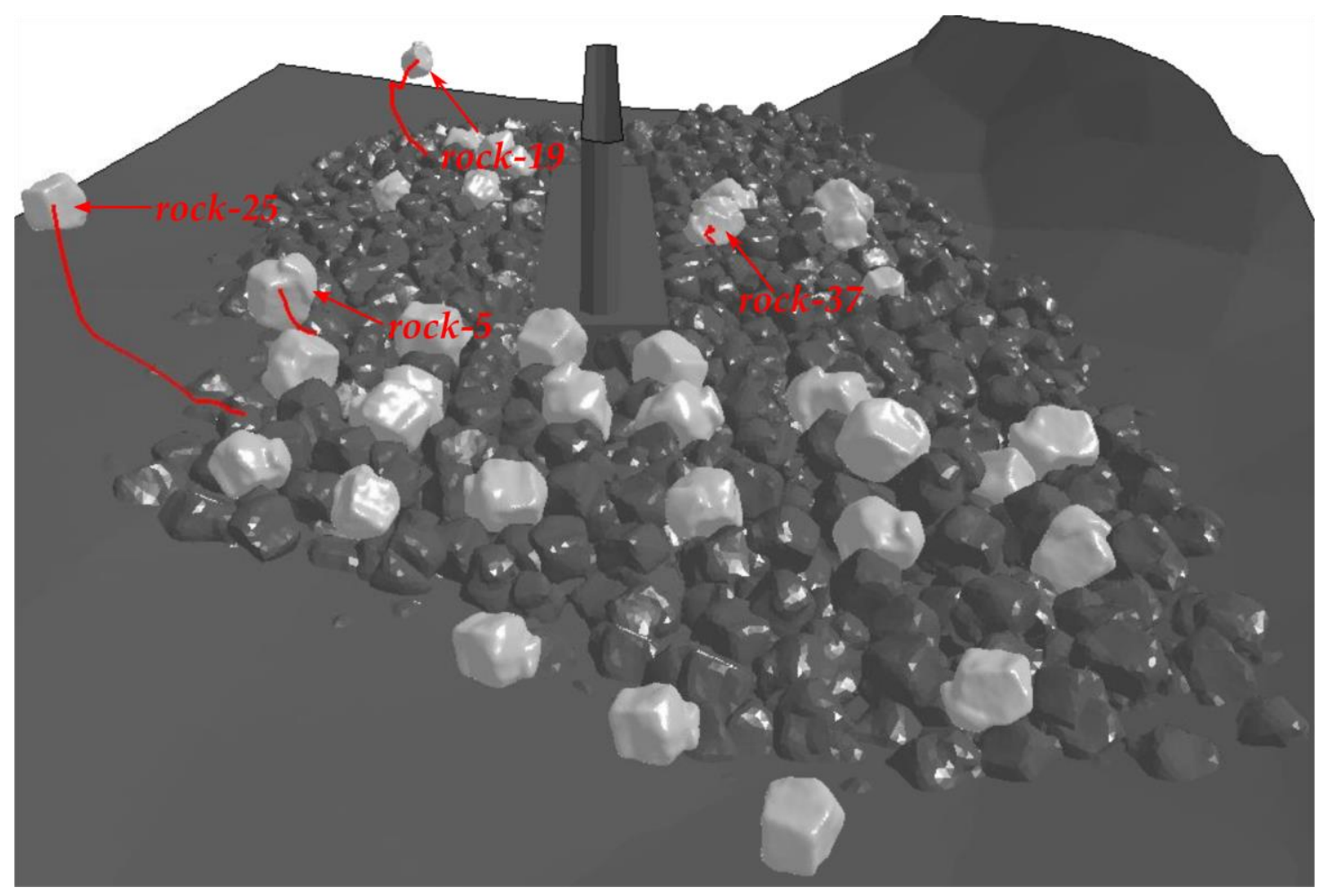

Figure 5.28: Trajectories of the moving rocks for the case Q100 discharge (11.1 ft/s average inlet velocity)

In order to determine under what conditions 1.0 T rocks start to move, several cases with higher inlet velocities into the scour sub-region were considered. It has to be noted that a scaling up the velocity on inlet faces by a factor of 1.5, for example, does not correspond necessarily to the specific conditions of a 150 year flood in the river. Not only may the magnitude of the velocity be different, but also the characteristics of the flow under the bridge might be different in these conditions if the entire river is modeled. Overtopping of the road and the bridge may also occur for the larger floods and that would change the velocity profile around the bridge. Nevertheless, the scaled up velocity analysis does provide useful engineering information about how close the riprap installation is to an onset of motion failure for the Q10o flood conditions.

Another important thing to note is that 3 dimensional CFD multi-phase simulations of flood events face a problem that can make it challenging to obtain a physically realistic solution in a reasonable amount of time. In VOF free surface simulations specific conditions are set at a flow inlet while fairly weak outlet boundary conditions are set, which are normally either a specified pressure or a zero gradient condition where the mass flow arriving at the outlet simply passes through with no velocity gradient as though it is a fully developed flow. A free surface or water elevation cannot be specified at an outlet. However, for subcritical flows, the flow in the domain is in fact controlled by the outlet conditions, most importantly the water elevation, that, while known, is not enforced by the software. This situation makes the specification of the inlet water level and initial conditions in the entire domain, including initial velocity and water level, fairly important for a number of reasons. A primary reason is that these flows are normally highly convective and information consequently propagates quickly downstream, but only propagates slowly from the outlet back to the inlet with the standard available numerical solvers. An initial 
condition that is too far from the final solution may cause, if the computation does not diverge, a sudden jump of the water level at the inlet boundaries and make the correctness of the solution in the domain questionable. Such behavior was observed when in the analysis of the entire domain the discharge was increased by $60 \%$. Figure 5.29 shows the water surface level over the entire domain in that simulation with water velocity color plotted on it. Figure 5.30 shows a zoomed view of the inlet boundary. A sudden increase of the water level by several feet can be observed. This problem is hard to overcome, especially considering the fact that such CFD simulations take several days on high performance cluster computers to converge to a quasi-steady state. An iterative approach can be used to adjust the water height at the inlet and the water height in the domain by changing the resistance of the flow in the domain (bed roughness, surface slope, and various solution parameters). For that reason the reduced domain analysis gives us more control over the inlet conditions and there is a simple relation between that velocity and velocities around the rocks in the domain.

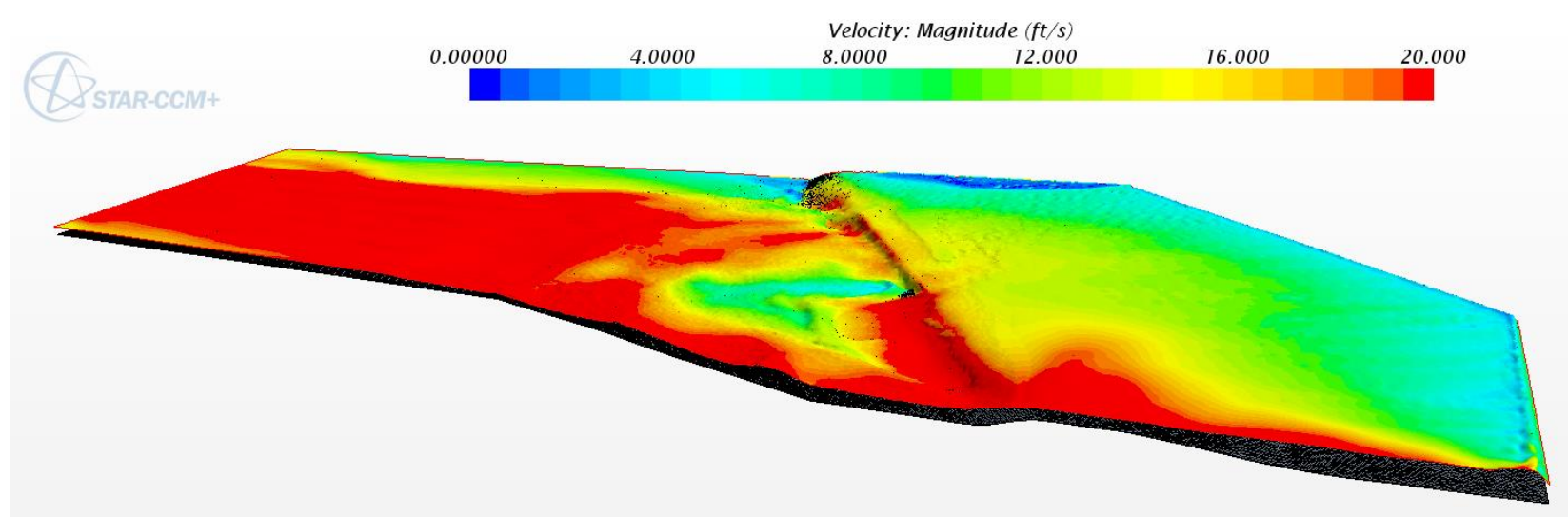

Figure 5.29: Water surface level in the simulation of $1.6 *$ Q10o conditions

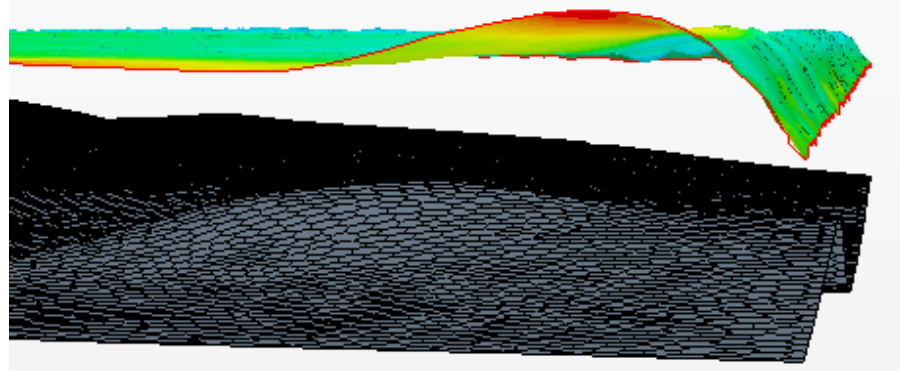

Figure 5.30: Raised water surface level by the inlet in the simulation of 1.6 * Q10o conditions

The next analyzed case was with the inlet velocities scaled by 1.1 as compared to the base case $\left(1.1^{*}\right.$ Q100). An initial CFD analysis was performed on the reduced domain in order to obtain quasi-steady state results, including converged values of the forces on the moveable rocks. Table 5.2 contains the forces on the three most nearly critical rocks in each weight category. Except for one rock, this is the same list as for the case of 1.0 * Q100. Rock 5 ( 1 Ton) this time was the fourth most critical among 1 Ton rocks and was replaced on the second position by Rock 8 . For some of the rocks the ratio of resultant force to the weight became very high. We already know from the previous analysis that some of these rocks will move even sooner, for lower velocities. Their behavior in simulations with higher inlet velocities may appear to be unrealistic as instead of rolling over the neighboring rocks they are leaving their positions at steeper angles. This behavior 
is expected and if the cost of FSI simulations wasn't prohibitively high, we would run just one long simulation where the velocity of the water is gradually increased, just how it is usually done in experiments. At the current state of computational power and software capabilities, a gradual ramping up of water velocity is too expensive to perform.

Table 5.2: Forces on the critical rocks in the simulation with average inlet velocity $12.2 \mathrm{ft} / \mathrm{s}(1.1$ * Q100)

\begin{tabular}{|c|c|c|c|c|c|}
\hline Rock \# & X Force & Y Force & Z Force & Resultant & Ratio to weight \\
\hline \multicolumn{6}{|c|}{1 Ton $(9.81 \mathrm{kN})$} \\
\hline 6 & -0.76 & 1.96 & 6.18 & 6.53 & 0.67 \\
\hline 8 & -0.39 & 3.08 & $5 \cdot 56$ & 6.37 & 0.65 \\
\hline 9 & -1.66 & 1.77 & 5.48 & 5.99 & 0.61 \\
\hline \multicolumn{6}{|c|}{$0.8 \operatorname{Ton}(7.85 \mathrm{kN})$} \\
\hline 19 & -0.30 & 3.53 & 6.25 & 7.18 & 0.92 \\
\hline 17 & -0.37 & 2.07 & 3.80 & 4.34 & 0.55 \\
\hline 13 & 0.18 & 1.35 & 4.14 & 4.36 & 0.56 \\
\hline \multicolumn{6}{|c|}{$0.6 \operatorname{Ton}(5.88 \mathrm{kN})$} \\
\hline 25 & -1.13 & 1.65 & 3.96 & 4.44 & 0.75 \\
\hline 26 & -0.32 & 1.22 & 4.08 & 4.27 & 0.73 \\
\hline 29 & -0.36 & 1.40 & 3.95 & 4.21 & 0.72 \\
\hline \multicolumn{6}{|c|}{$0.4 \operatorname{ton}(3.92 \mathrm{kN})$} \\
\hline 35 & 0.47 & 1.45 & 3.25 & 3.59 & 0.92 \\
\hline 39 & -0.11 & 1.06 & 3.15 & $3 \cdot 33$ & 0.85 \\
\hline 40 & 0.17 & 1.44 & 2.52 & 2.91 & 0.74 \\
\hline
\end{tabular}

In the FSI simulation, motion of 6 rocks was detected for that case. Five of them moved significantly away from their initial positions within the simulated time. The following rocks experienced motion: rock $5(1 \mathrm{~T})$, rock $19(0.8 \mathrm{~T})$, rocks 25,27 , and $29(0.6 \mathrm{~T})$. Rock $37(0.4 \mathrm{~T})$ moved locally. Figure 5.31 shows several snapshots from that simulation. This result again shows that the rocks with the highest forces acting on them are not necessarily the ones that will move first because gravity and the reaction forces of surrounding rocks also play and import role in determining whether the flow force on a rock is sufficient to move it out of position. Out of the 1 Ton rocks, again only one rock was unstable. Figure 5.32 shows the trajectories of center of gravity for the moving rocks. 


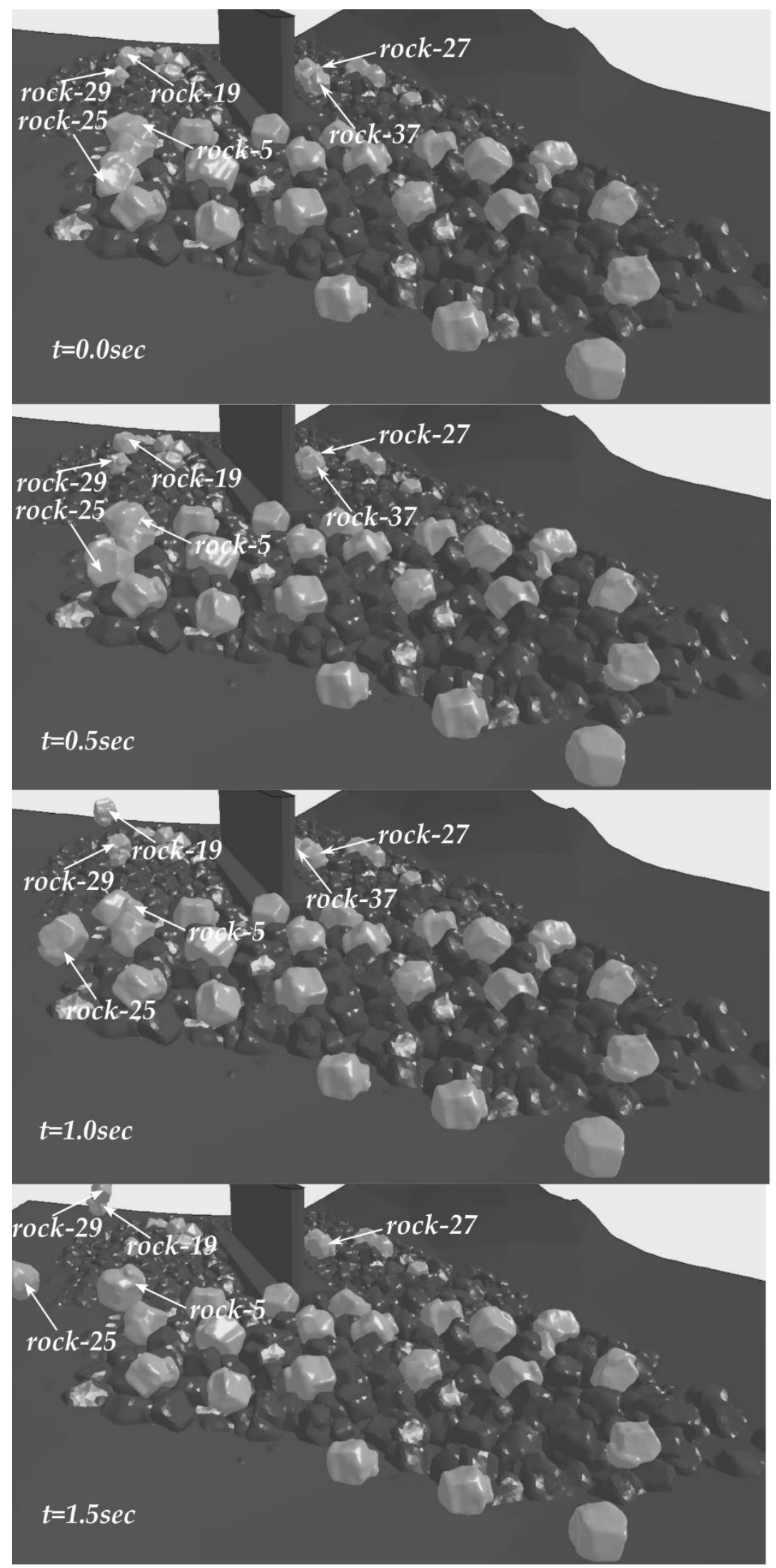

Figure 5.31: Snapshots of the FSI simulation for $1.1{ }^{*}$ Q100 discharge (12.2 ft/s average inlet velocity) 


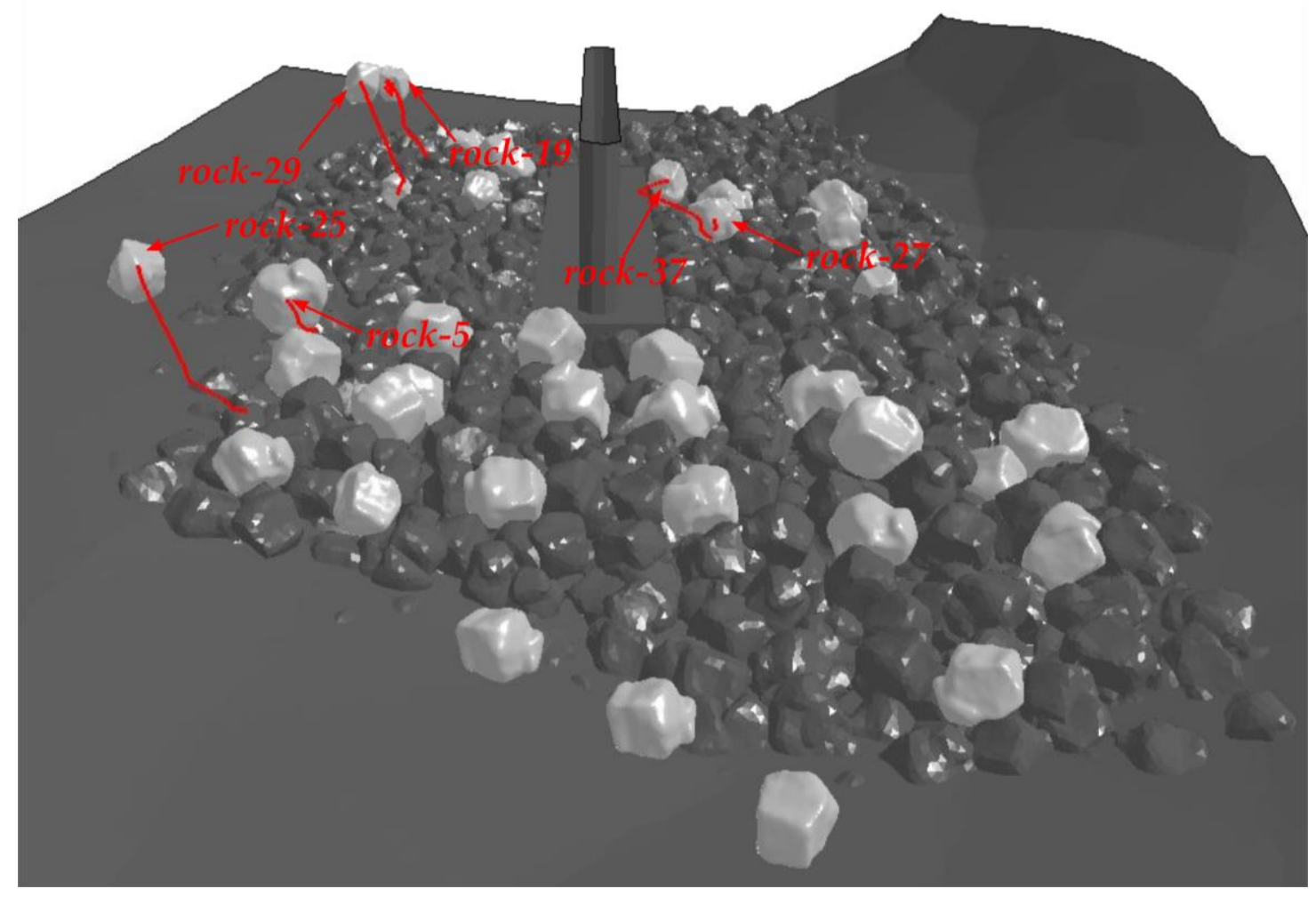

Figure 5.32: Trajectories of the moving rocks for the case $1.1{ }^{*}$ Q100 discharge $(12.2 \mathrm{ft} / \mathrm{s}$ average inlet velocity)

In the next case, the velocities on the inlet were multiplied by a factor of $1.2\left(1.2{ }^{*} \mathrm{Q} 100\right)$ to an average value of $13.3 \mathrm{ft} / \mathrm{s}$. The faster the flow and the more moving rocks, the more problems there are with the FSI simulations. The mesh around the moving and colliding rocks experiences drastic deformations and the simulations fail frequently. Manual intervention is required in order to restart the analysis with modified mesh conditions to push the simulation forward. This case was only run to slightly above 1.0 sec of real time. 
Table 5.3 shows maximum forces on three rocks within each weight group. For some of the rocks the ratio of the resultant force to their weight is very close to 1 . That means if they are not blocked by other rocks around them, they will definitely be displaced from their initial positions. The FSI analysis indicates that nine rocks have moved. The simulation wasn't long enough to say for certain that more of them wouldn't move but within one second of simulated time they were not moving. Only one rock with a weight of one tone has moved, rock 5. Also only one rock with weight of $0.8 \mathrm{~T}$, rock 19, was set in motion. Four rocks weighing $0.6 \mathrm{~T}$ have moved, rocks $25,26,27$, and 29. Two rocks weighing $0.4 \mathrm{~T}$ have moved, rock 35 and rock 37. Figure 5.33 shows the trajectories of center of gravity for the moving rocks. 
Table 5.3: Forces on the critical rocks in the simulation with average inlet velocity $12.2 \mathrm{ft} / \mathrm{s}(1.1 *$ Q10o $)$

\begin{tabular}{|c|c|c|c|c|c|}
\hline Rock \# & X Force & Y Force & Z Force & Resultant & Ratio to weight \\
\hline \multicolumn{6}{|c|}{1 Ton $(9.81 \mathrm{kN})$} \\
\hline 6 & -0.39 & 2.55 & 6.60 & 7.09 & 0.72 \\
\hline 5 & -1.11 & 3.41 & 5.57 & 6.62 & 0.68 \\
\hline 8 & -0.49 & 3.46 & 5.40 & 6.43 & 0.66 \\
\hline \multicolumn{6}{|c|}{0.8 Ton $(7.85 \mathrm{kN})$} \\
\hline 19 & -0.78 & 3.77 & 6.66 & 7.69 & 0.98 \\
\hline 17 & -0.36 & 2.41 & 3.81 & 4.52 & 0.58 \\
\hline 13 & 0.29 & 1.51 & 4.03 & $4 \cdot 31$ & 0.55 \\
\hline \multicolumn{6}{|c|}{$0.6 \operatorname{Ton}(5.88 \mathrm{kN})$} \\
\hline 29 & -0.58 & 1.66 & 4.36 & 4.70 & 0.80 \\
\hline 25 & -1.50 & 1.77 & 4.03 & 4.65 & 0.79 \\
\hline 26 & -0.46 & 1.34 & 4.37 & 4.59 & 0.78 \\
\hline \multicolumn{6}{|c|}{$0.4 \operatorname{ton}(3.92 \mathrm{kN})$} \\
\hline 35 & 0.55 & 1.70 & 3.64 & 4.05 & 1.03 \\
\hline 39 & -0.62 & 1.24 & 3.50 & 3.76 & 0.96 \\
\hline 40 & 0.17 & 1.82 & 2.96 & 3.48 & 0.89 \\
\hline
\end{tabular}

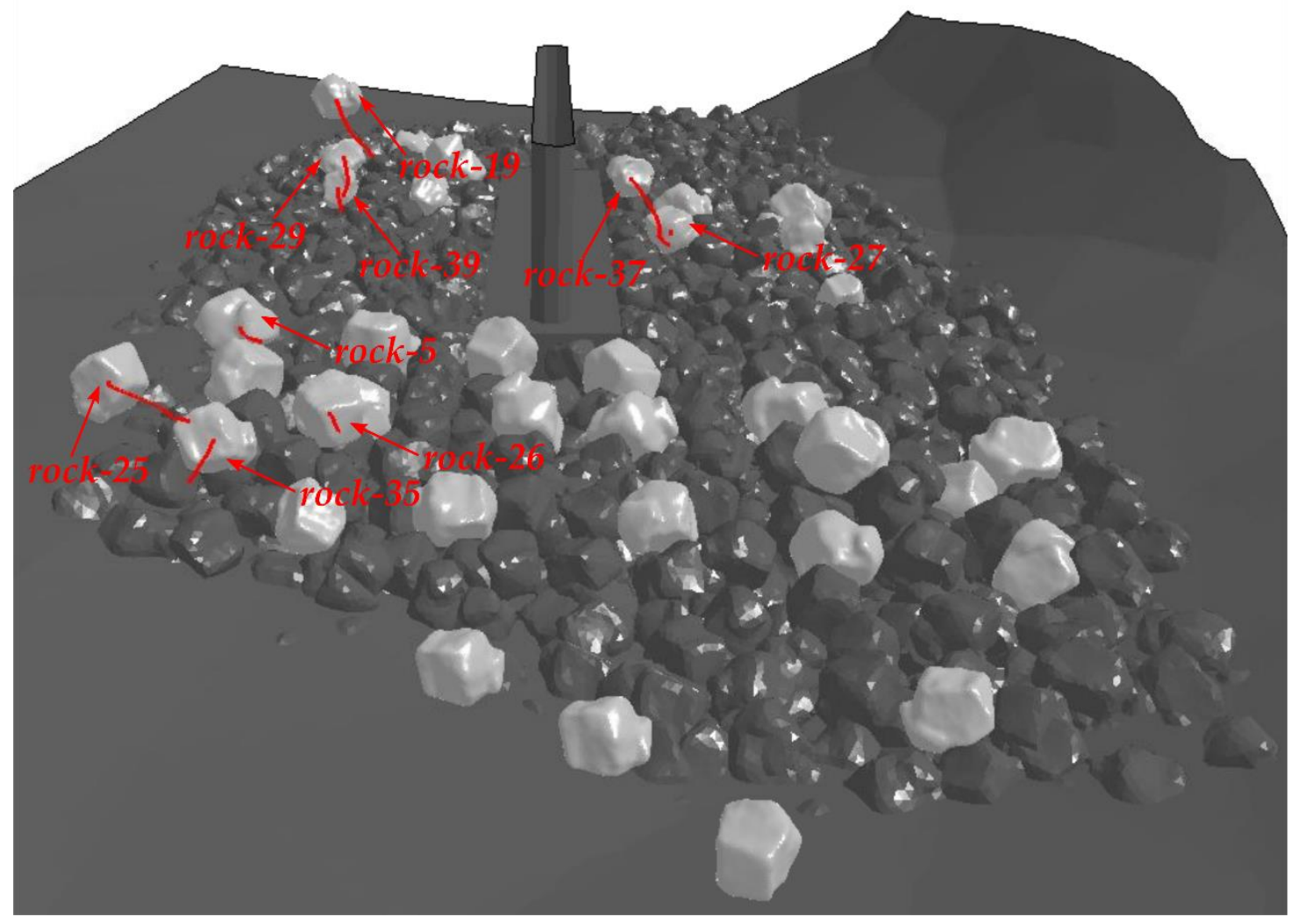

Figure 5.33: Trajectories of the moving rocks for the case $1.2{ }^{*}$ Q100 discharge $(13.3 \mathrm{ft} / \mathrm{s}$ average inlet velocity) 


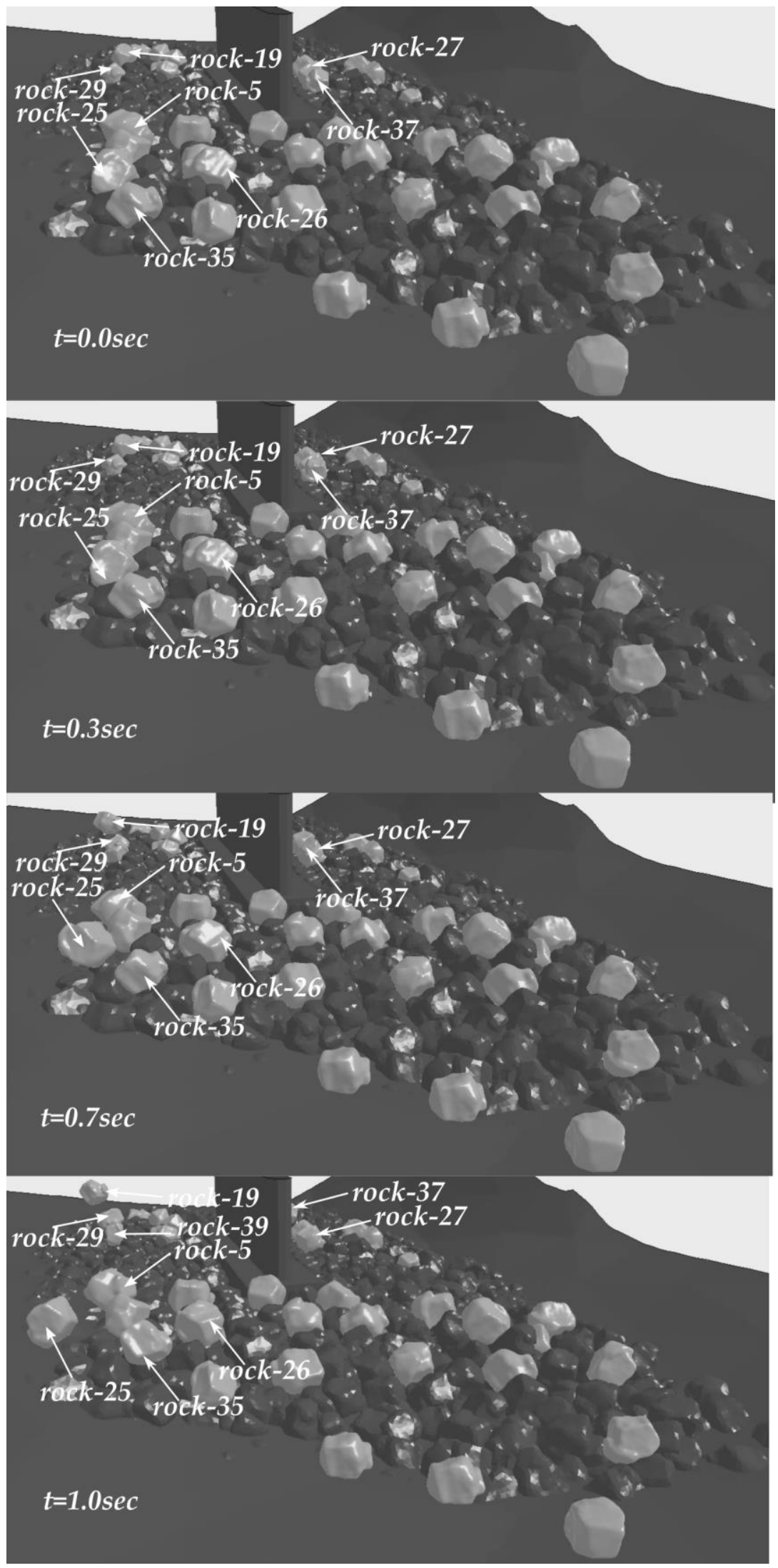

Figure 5.34: Snapshots of the FSI simulation for $1.2 *$ Q100 discharge (13.3 ft/s average inlet velocity) 


\section{Summary and Conclusions}

A new advanced computational methodology for assessing failure risk of geometrically complex riprap installations or planned installations, including failure risk based on riprap size and shape, such as those found in field conditions has been developed. In this method, the detailed flow force distribution on riprap rock surfaces including both pressure and local shear on the solid surface is computed using the $3 \mathrm{D}$ CFD software STAR-CCM+. The CFD software is coupled through file data exchange to the computational structural mechanics software LS-DYNA, and the flood flow threshold for onset of motion of riprap rocks is computed for a medium to large set of representative riprap rocks that are free to move with motion tracked by the software. The entire irregular surface of a riprap installation is meshed out in detail in the computational domain down into the second layer of riprap or more in order for flow in the interstitial spaces between rocks to be computed with the consequence force distribution on the lower sides and undersides of the representative movable rocks. The representative movable rocks are distributed in the riprap geometry in positions where velocities are likely to be highest or position, such as at the toe, is likely to expose the rock to more flow force or flow force without constraining neighbors on all sides.

This methodology solves the onset of motion analysis problem as a loosely (explicit) coupled fluid structure interaction, FSI, problem. While using tight (implicit) coupling and making all of the rocks in the riprap installation movable is conceptually possible and would be more accurate, that approach would be far too expensive and time consuming for reasonable engineering application to real field problems. As detailed in the report, the explicit coupling is conservative and the procedure for assessing riprap failure risk and failure conditions for the specific geometry of a field riprap installation constitutes a major step forward in providing the capability to determine if an existing or planned riprap installation will be adequate.

The work included analysis of both a laboratory experiment where the onset of motion conditions were tested and result data was available, and an actual full scale field case, the Middle Fork Feather River Bridge, which is located on State Route 89 in Plumas County, California near the town of Blairsden and Graeagle.

The laboratory experiment was analyzed at full scale, and the experimental results were scaled up for comparison with the analysis. Conservative qualitative agreement was demonstrated between the experiment and the simulations. Given the engineering simplifications needed to run the analysis in a two week period after problem setup, the agreement between analysis and experiment is quite good and safe because it was conservative, under-predicting the velocity for onset of riprap motion for a given rock size.

The modeling, analysis, and assessment of field sites, the Middle Fork Feather River Bridge case in this work, adds additional complexities that are often not present in well controlled laboratory experiments. Several observations can be made regarding field site conditions and the Feather River analysis results:

- The $3 \mathrm{D}$ scan of the riprap installation was useful in defining the boundaries of the riprap at the site, but the data from the scan could not be used directly in CAD software to

Page 72 
generate the bed geometry of the computational domain because the scans do not capture hidden interstitial spaces between riprap rocks.

- The planned design, including riprap size, may not match the as built condition. Pictures and scans at the Feather River site show that the planned 1 ton $D_{50}$ rock contained a large number of smaller rocks. Consequently, the analysis was carried out with rocks that varied in weight from 0.4 ton to 1 ton.

- Only a couple of the rocks were set in motion for the Q10o flood analysis.

- Analysis using the Q10o flood velocity increased by $10 \%$ more than doubled the number of rocks set in motion to 5 , and a case with velocity increased by $20 \%$ set eight rocks in motion, including 1 ton and smaller rocks. These results indicate that the current riprap size at the installation is close to minimum for stability of the installation in Q10o floods.

- The FSI analysis showed that onset of motion did not always occur for the rocks with the highest flow force to weight ratio. The reaction forces that arise from the arrangement and position of rocks with respect to their neighbors also plays a significant role in onset of motion. Therefore, the extent of interlocking in the arrangement and how well it constrains onset of motion, needs to be considered, as well as the flood flow velocity in the sizing of riprap.

The cost of applying the methodology is certainly much more expensive than a relatively quick calculation using any of the existing riprap sizing formulas, the amount of geometric detail and fundamental flow and rigid body physics applied in the new methodology is vastly greater. Therefore, good candidate applications for using FSI analysis to assess riprap installation would be those where the project cost is much higher and therefore it is worth spending a small fraction of the project funds to raise the confidence level that the riprap installation will keep the bridge foundations safe. New riprap projects or existing installations where the failure cost is much higher than the analysis cost are also good candidates for this type of advanced assessment methodology. 


\section{7. $\quad$ References}

[1] Flora K., Advanced Hydraulic Report for Pier Riprap Stability, Middle Fork Feather River Br. N. 09-0063, California Department of Transportation, June, 2014

[2] Lagasse P.F., Clopper P.E., Pagán-Ortiz J.E., Zevenbergen L.W., Arneson L.A., Schall J.D., Girard L.G., HEC-23 Bridge Scour and Stream Instability Countermeasures: Experience, Selection, and Design Guidance-Third Edition, Publication No. FHWA-NHI09-111, U.S. Department of Transportation, 2009.

[3] Lagasse P.F., Clopper P.E., Zevenbergen L.W., Ruff J.F., NCHRP Report 568 Riprap Design Criteria, recommended Specifications, and Quality Control, Transportation Research Board, Washington, DC, 2006.

[4] J. Sterling Jones Hydraulics Research Laboratory, https://www.fhwa.dot.gov/research/tfhrc/labs/hydraulics/, Accessed: February 7, 2017.

[5] MeshLab, http://www.meshlab.net/, Accessed: February 7, 2017.

[6] SIEMENS User Guide STAR-CCM+ Version 10.062, 2016.

[7] Livermore Software Technology Corporation (LSTC), LS-DYNA $®$ Keyword User's Manual Version 971, LSTC, 2007.

[8] Duarte F., Gormaz R., Natesan S., Arbitrary Lagrangian Eulerian method for Navier Stokes equations with moving boundaries, Methods Appl. Mech. Engrg., Vol.193, pp. 4819-4836, 2004.

[9] Hou G., Wang J., Layotn A., Numerical Methods for Fluid Structure Interaction - A Review, Commun. Comput. Phys., Vol. 12, No. 2, pp. 337-377, 2012.

[10] Livermore Software Technology Corporation (LSTC), Incompressible fluid solver in LSDYNA, LSTC, 2013.

[11] Kerenyi K., Jones J., Stein S., Bottomless Culvert Scour Study: Phase II Laboratory Report, FHWA-HRT-07-026, U.S. Department of Transportation, 2007.

[12] Pagan-Otiz J.E., Stability of Rock Riprap for Protection at the Toe of Abutments Located at the Floodplain, Publication No. FHWA-RD-91-057, September, 1991 


\section{Argonne}

\section{Energy Systems Division}

Argonne National Laboratory

9700 South Cass Avenue, Bldg. 362

Argonne, IL 60439-4815

www.anl.gov

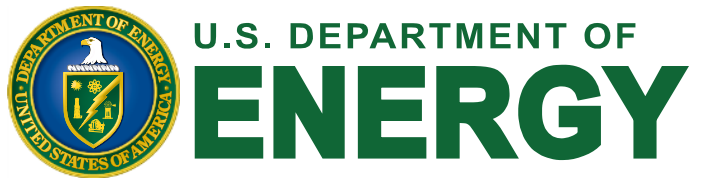

Argonne National Laboratory is a U.S. Department of Energy laboratory managed by UChicago Argonne, LLC 